\author{
Universidade de São Paulo \\ Instituto de Física
}

\title{
MODELOS ESTATÍSTICOS PARA A TRANSIÇÃO ORDEM- DESORDEM DE CAMADAS LIPÍDICAS
}

\section{HENRIQUE SANTOS GUIDI}

\author{
Tese de doutorado apresentada ao Instituto de Física para a \\ obtenção do título de Doutor em Ciências
}

Orientadora: Prof ${ }^{a}$. Dr ${ }^{\mathrm{a}}$ Vera Bohomoletz Henriques

Banca Examinadora:

Prof ${ }^{\mathrm{a}}$. Dr ${ }^{\mathrm{a}}$ Vera Bohomoletz Henriques

Prof. Dr. Mário José de Oliveira (IFUSP)

Vie B. Hen Y

Prof. Dr. Cristiano Luis Pinto de Oliveira (IFUSP)

Prof. Dr. Alexandre Diehl (UFPEL)

Prof. Dr. Bismarck Vaz da Costa (UFMG)

São Paulo

2012 


\section{FICHA CATALOGRÁFICA}

Preparada pelo Serviço de Biblioteca e Informação do Instituto de Física da Universidade de São Paulo

Guidi, Henrique Santos

Modelos estatísticos para a transição ordem-desordem de camadas lipídicas. São Paulo, 2013.

Tese (Doutorado) - Universidade de São Paulo. Instituto de Física, Depto. Física Geral

Orientador: Profa. Dra. Vera Bohomoletz Henriques

Área de Concentração: Física

Unitermos: 1.Biofísica; 2. Física teórica; 3. Mudança de fase; 4.Física computacional. 


\section{AGRADECIMENTOS}

Agradeço à professora Vera pela paciência, companherismo e competência durantes vários anos de orientação. Ao professor Eduardo Henriques pelas muitas conversas construtivas e por observar a possibilidade do nosso modelo descrever monocamadas de Langmuir. À professora Maria Teresa Lamy, Thais Enoki e o grupo de biofísica do IFUSP por serem tão interativos e acolhedores.

Aos professores, funcionários e colegas, que fazem a universidade funcionar. Aos meus pais, que me apoiaram.

Aos meus amigos, que de alguma forma, inclui muitos já citados.

Ao CNPq, pelo apoio financeiro. 
"A luta contra o erro tipográfico tem algo de homérico. Durante a revisão os erros se escondem, fazem-se positivamente invisíveis. Mas assim que o livro sai, tornam-se visibilíssimos..." (Monteiro Lobato) 


\section{RESUMO}

Lipídios em solução aquosa formam uma variedade de estruturas diferentes que incluem monocamadas de surfactantes na interface água-ar, conhecidas como monocamadas de Langmuir, como também vesículas unilamelares ou plurilamelares no interior da solução. Sob variação de temperatura, estas estruturas apresentam diferentes fases, observadas através de calorimetria ou variação isotérmica de pressão lateral. Entre as fases apresentadas por estas estruturas, as duas mais importantes se diferenciam pela ordem das cadeias lipídicas. Entendemos que do ponto de vista das fases termodinâmicas, simplificado e qualitativo, monocamadas de Langmuir e bicamadas lipídicas constituem o mesmo sistema físico sob vínculos diferentes.

Neste trabalho, desenvolvemos um modelo estatístico para o estudo da transição ordem-desordem destes sistemas, que inclui flutuações de densidade, estas ausentes no modelo de Doniach, de 1980, a base para muitos estudos teóricos para transições de fase de sistemas lipídicos. Flutuações de densidade são fundamentais na descrição de vesículas lipídicas carregadas, compostas de surfactante cuja cabeça polar se dissocia em água. O estudo em laboratório das propriedades térmicas e estruturais de membranas artificiais de lipídios carregados é relativamente recente, e foi desenvolvido em grande parte no Laboratório de Biofísica do IFUSP. Tais membranas apresentam comportamento distinto das membranas neutras, notoriamente influenciado pela concentração de sal na solução. Isto motivou o desenvolvimento de uma segunda versão do modelo, na qual passamos a descrever a cabeça polar do lipídio em termos de um par de cargas opostas, sendo que a camada lipídica foi acoplada ao modelo primitivo restrito na rede, que desempenha o papel da solução salina.

O primeiro modelo foi estudado por aproximação de campo médio e por simulações de Monte Carlo, e o segundo modelo foi investigado apenas através de simulações numéricas. O estudo do modelo carregado foi precedido por uma investigação criteriosa das técnicas de simulação de sistemas com interação Coulombiana, resultando no desenvolvimento de uma metodologia adequada a condições de contorno não isotrópicas e com custo computacional reduzido.

Os modelos estatísticos propostos por nós levaram a dois resultados importantes. O modelo para camadas lipídicas neutras é, até hoje, o único modelo estatístico que descreve tanto a transição gel-fluido de bicamadas lipídicas, como a transição "líquido condensado" - "líquido expandido" de monocamadas de Langmuir, além de descrever também a transição "líquido expandido"-gás na interface água-ar. O modelo para camadas lipídicas que se dissociam em água reproduz a variação abrupta na dissociação, concomitante com a transição ordem-desordem, propriedade que permite interpretar estudos experimentais relativos à condutividade das soluções lipídicas correspondentes. 


\begin{abstract}
Lipids in aqueous solution form a variety of different structures which include monolayers of surfactants at the water-air interface, known as Langmuir monolayers, as well as unilamellar or plurilamellar vesicles within the solution. Under temperature variation, these structures display different phases, observed through calorimetry or isothermal variation of lateral pressure. Among the phases presented by these structures, the two most important differ in the order of the lipid chains. From the point of view of the thermodynamic phases, our understanding is that Langmuir monolayers and lipid bilayers constitute the same physical system under different constraints.

In this work, we develop a statistical model for the order - disorder transition of lipid bilayers which adds density fluctuations to Doniach's 1980 model, which has been considered the basis for many theoretical studies for lipid systems phase transitions. Density fluctuations are essential in the description of the properties of charged vesicles in solution, which consist of surfactants whose polar head dissociates in water. The study in the laboratory of thermal and structural properties of artificial charged lipid membranes is relatively new, and was developed largely in the IFUSP Laboratory of Biophysics. Such membranes exhibit distinct behavior if compared to neutral membranes, notoriously influenced by the solution salt concentration. The experimental investigations motivated us to develop a second model, in which we describe the polar headgroups through a pair of opposite charges. The lipid layer is attached to the lattice restricted primitive model, which plays the role of the saline solution.

The first model was studied both through a mean-field approximation as well as through Monte Carlo simulations, whereas the second model was investigated only through numerical simulations. The study of the charged model was preceded by a thorough investigation of the simulation techniques for Coulomb interaction sytems, leading to the development of a methodology suitable for non isotropic boundary conditions and with reduced computational cost.

The statistical models proposed by us led to two important results. To our knowledge, our model for neutral lipid layers is the only statistical model which, aside from describing simultaneously both the gel-fluid transition of lipid bilayers and the condensed liquid - expanded liquid transition of Langmuir monolayers, also describes the gas- expanded liquid transition at the air-water interface. The model for lipid layers that dissociate in water reproduces the abrupt change in dissociation, concomitant with the order-disorder transition, a property that allows us to interpret experimental studies related to conductivity of the corresponding lipid solutions.
\end{abstract}




\section{Sumário}

1 Introdução 1

2 Modelos Estatísticos $\quad 17$

2.1 Modelo de Doniach . . . . . . . . . . . . . . . . . . 17

2.2 Introduzindo flutuações de densidade: gás de rede de Doniach $(\mathrm{DLG}) \ldots \ldots \ldots \ldots \ldots$

2.3 Modelo para a solução iônica: modelo primitivo restrito na

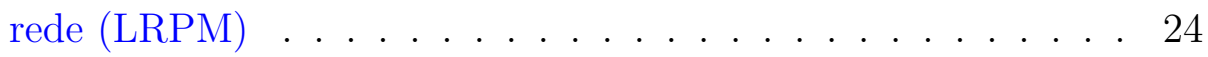

2.4 Membranas com cargas: DLG com cargas (CDLG) . . . . . 25

3 Modelo DLG: abordagem de Campo Médio 27

3.1 Aproximação de Campo Médio . . . . . . . . . . . . . . 28

3.2 Fases ordenadas a temperatura nula . . . . . . . . . . . . 34

3.3 Métodos numéricos . . . . . . . . . . . . . . . . . . . 39

3.4 Diagrama de fases . . . . . . . . . . . . . . . . . . . . . . 41

4 Modelo DLG: simulações numéricas $\quad 57$

4.1 Enumeração Exata . . . . . . . . . . . . . . . . . . 58

4.2 Método de Metropolis na presença de estados degenerados . . 61 
4.3 Cálculo eficiente das diferenças de "energia": $\Delta E$ e $\Delta \phi \ldots . .65$

4.4 Ensemble Grande canônico . . . . . . . . . . . . . . . . . . . . 69

4.5 Ensemble Canônico . . . . . . . . . . . . . . . . . . . . . . 74

4.5.1 Método de Widom e enumeração exata . . . . . . . . . 74

4.5.2 Resultados da simulação no ensemble canônico . . . . . 77

5 Solução salina e o modelo LRPM 85

5.1 Definição do modelo LRPM . . . . . . . . . . . . . . 86

5.2 Convergência da energia eletrostática . . . . . . . . . . 87

5.3 Condições de contorno . . . . . . . . . . . . . . . . . . . . . 89

5.4 Métodos para o cálculo da energia eletrostática . . . . . . . . 95

5.4 .1 Soma de Ewald . . . . . . . . . . . . . . . . 95

5.4.2 Cálculo direto versus Ewald: a constante de Madelung 97

5.4.3 Cálculo direto versus Ewald para líquidos . . . . . . . . 99

5.4.4 Importância da correção de dipolo . . . . . . . . . . . 103

5.5 Cálculo eficiente da diferença de energia: a função $f\left(\mathbf{r}_{i j}\right)$. . . 105

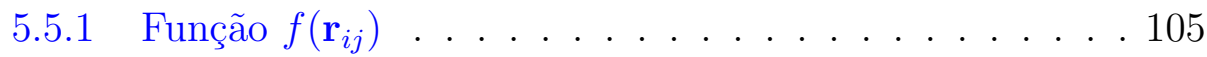

5.5.2 Cálculo da diferença de energia . . . . . . . . . . 108

5.6 Comentários sobre custo computacional e precisão numérica 112

6 Modelo para transição gel-fluido em membranas carregadas em solução aquosa

6.1 Construção do modelo . . . . . . . . . . . . . . . . 116

6.2 Teste do modelo: plano carregado e solução iônica . . . . . . . 118

6.3 Resultados exploratórios: solução lipídica sem sal . . . . . . 124 
7 Conclusões e considerações finais 


\section{Capítulo 1}

\section{Introdução}

A membrana celular envolve todas as células, é seletivamente permeável e contém uma grande variedade de moléculas biológicas, envolvidas em diversos processos celulares, como a adesão celular, condutância do canal iônico e sinalização celular $[1,2]$. Existem evidências que sugerem que estas funções celulares estejam relacionados com a fluidez da membrana. Então, há um considerável interesse em se entender os vários parâmetros que controlam a fluidez, tais como composição, pH, campo elétrico e, principalmente, a temperatura [3].

Membranas naturais são formadas com lipídios diferentes que podem se dissociar em água ou em soluções tampão [4], como o sangue. Vamos nos restringir às membranas artificiais, feitas em laboratório, formadas por uma única espécie de lipídio. Lipídios consistem de uma cabeça hidrofílica e duas caudas hidrofóbicas. Em solução aquosa, lipídios tendem a formar estruturas de maneira tal que as caudas hidrofóbicas não entrem em "contato" com a água. Na superfície da solução ocorre a formação de uma monocamada de 
Figura 1.1: Desenho de algumas formas de auto organização de lipídios em solução: o lipossomo (Liposome), a micela (Micelle) e a bicamada (Bilayer sheet) [5]. Os lipídios formadores dos lipossomos ocupam o espaço equivalente a um cilindro. Os lipídios com cadeias de carbono curtas e os detergentes, que possuem uma única cadeia, são cônicos e propícios para a formação de micelas

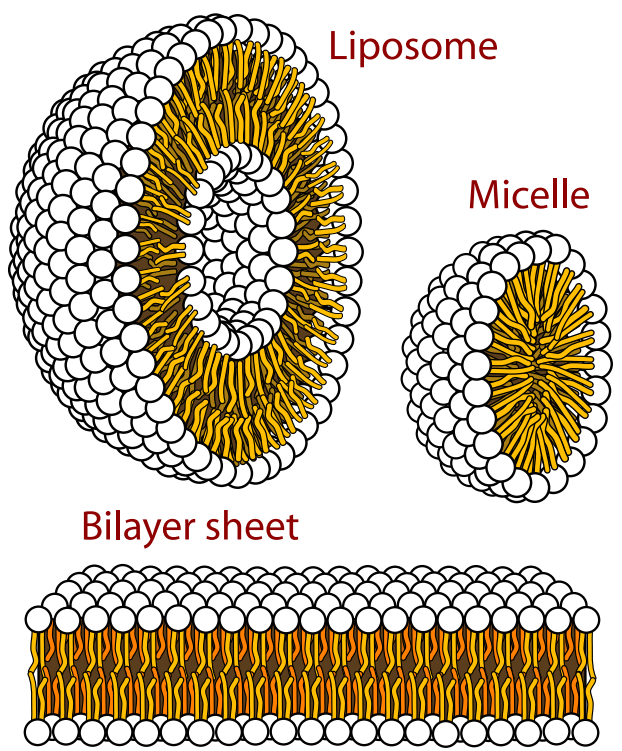

lipídios, conhecida por monocamada de Langmuir, e, no interior da solução, podem formar diferentes estruturas, exemplificadas na figura 1.1. A forma mais simples é chamada de vesícula unilamelar (ou lipossomo), constituindo uma única bicamada que se fecha em si mesma, formando uma esfera oca.

Lipídios são classificados em função da composição das suas cabeças em combinação com a composição e tamanho das caudas. Independentemente da composição da cabeça polar dos lipídios que constituem a membrana em solução aquosa, reconhece-se pelo menos duas fases termodinâmicas distintas: a fase gel e a fase fluida. A transição gel - fluido, sob a variação da temperatura, é caracterizada pelo aumento da mobilidade dos lipídios na superfície da membrana, pelo aumento da área superficial da membrana e por um pico estreito no calor específico. Estes parâmetros são medidos em laboratório, e, como exemplo, são apresentados, na figura 1.2a, resultados de ressonância paramagnética eletrônica (EPR) que indicam o aumento abrupto da mobilidade com a temperatura, para uma solução do lipídio difosfatidil- 


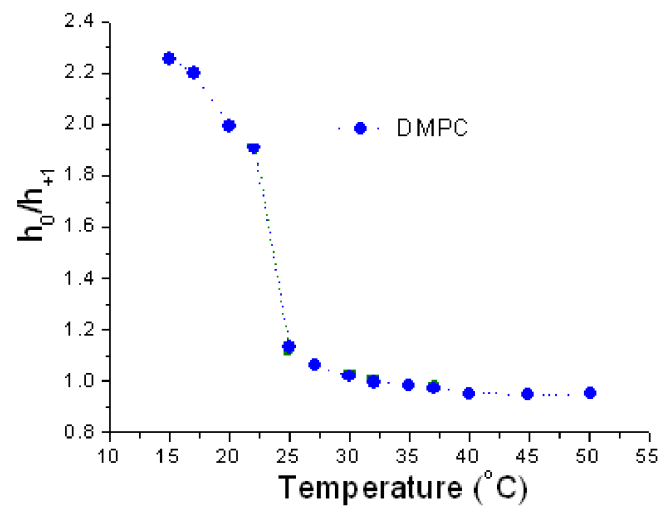

(a) EPR

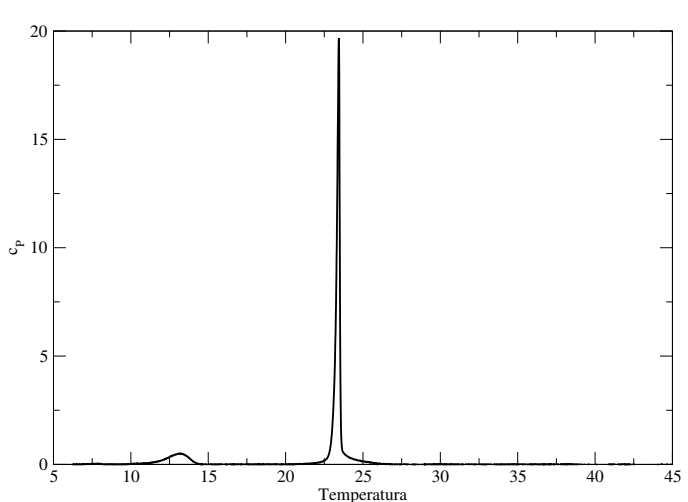

(b) Calor específico

Figura 1.2: Alguns resultados experimentais de vesículas contruídas com uma espécie de lipídio que não se dissocia em agua. (a) Resultado de espectroscopia por EPR de solução de vesículas de DMPC (difosfatidildimiristoil colina) indica que há um aumento de mobilidade das cadeias em $T=T t \approx 23^{\circ} \mathrm{C}$ [4]. (b) Calor específico a pressão constante, para vesículas construídas com DMPC, apresentando um pico na mesma temperatura onde ocorre o aumento da mobilidade das cadeias [6]

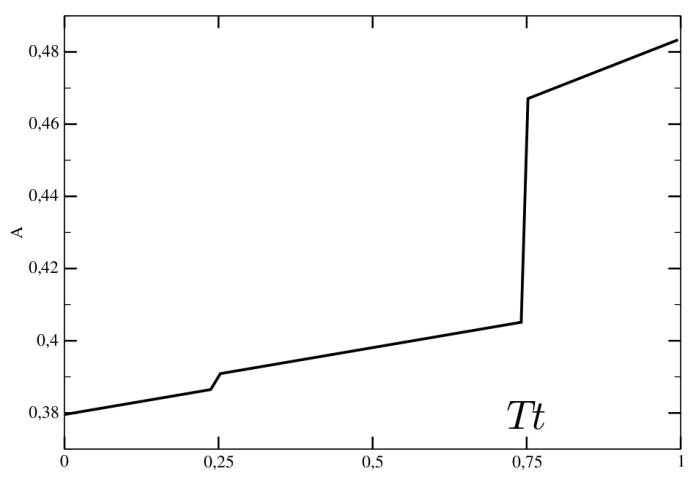

Figura 1.3: Curva esquemática do aumento de área por cabeça polar em função da temperatura para bicamadas. $T_{t}$ é a temperatura de transição gel-fluido. Cópia da figura 8.3 da referência [7].

dimiristoil colina (DMPC); na figura 1.2b um pico no calor específico para uma solução do mesmo lipídio, ambos obtidos no laboratório de biofísica do IFUSP. A figura 1.3 ilustra de forma esquemática o aumento da área da superfície da membrana, na temperatura de transição. Como os lipídios são orientados perpendicularmente à superfície e apresentam mobilidade restrita 
Figura 1.4: $\quad$ Figura esquemática das isotermas de monocamadas de Langmuir, cópia da referencia [8]. A fase liquido expandido (liquid expanded) é equivalente à fase fluido e as fases condensadas (tilted condensed e untilted condensed) são equivalente a fase gel.

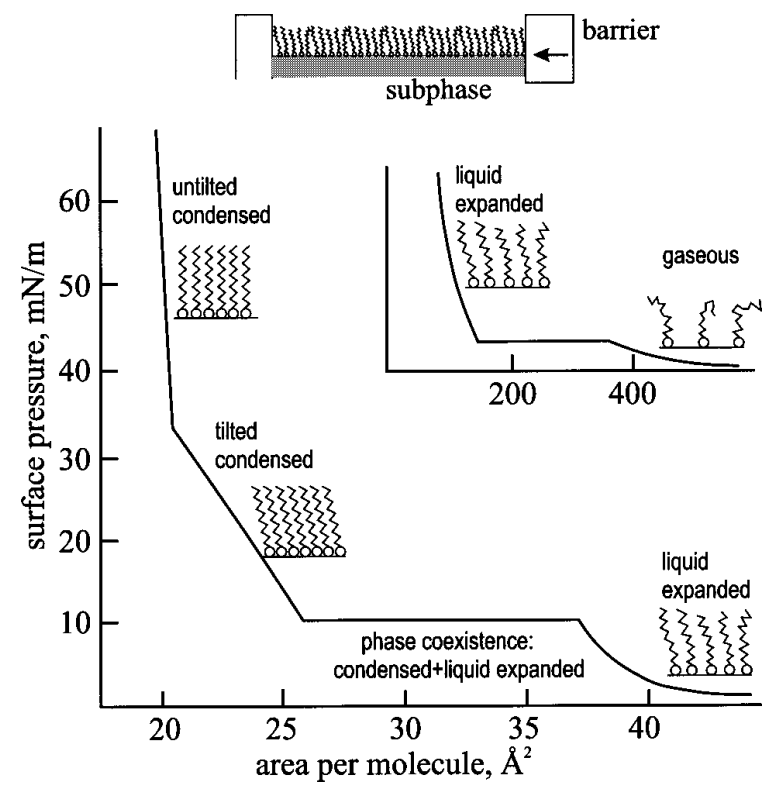

à superfície da vesícula, a fase fluida também é, ou era, identificada como uma fase cristal - líquido.

Os estudos experimentais levaram à hipótese de que na fase gel os lipídios encontram-se com suas caudas hidrofóbicas ordenadas, resultando em pequena área por cabeça polar, ao passo que na fase fluida as cadeias hidrocarbônicas desordenam-se, ocasionando um afastamento dos lipídios entre si e em um aumento da área ocupada por cabeça polar na superfície da membrana.

O estudo das propriedades da monocamada de lipídios na interface água - ar constitue uma área independente de pesquisa, a área de monocamadas de Langmuir. Foi verificada a existência de diferentes fases fluidas da monocamada, sob a variação da pressão lateral. As fases diferenciam-se em termos da densidade superficial, que sofre descontinuidade na transição de uma fase para a outra. A figura 1.4 ilustra uma isoterma de Langmuir, na qual apa- 
recem diversas fases, inclusive uma fase de alta pressão, a fase "condensada sem inclinação", que não é de nosso interesse neste estudo. Identificam-se uma fase de muito baixa densidade, denominada gasosa, e duas fases condensadas de diferentes densidades, às quais associa-se a presença de cadeias ordenadas ou desordenadas. As duas fases são denominadas fase de "líquido expandido" e fase de "líquido condensado".

Esta descrição qualitativa e microscópica das fases liquidas é equivalente à descrição das fases de vesículas lipídicas em solução. A fase gel de membranas equivale à fase condensada de monocamadas, e à fase fluida equivale a fase liquido expandido[8].

Nas duas últimas décadas, houve um grande investimento no estudo das propriedades termodinâmicas de membranas carregadas em solução [4], em particular, pelo grupo de biofísica do IFUSP. Este grupo vem investigando sistematicamente as propriedades de soluções do lipídio dimiristoilfosfatidil glicerol (DMPG), um lipídio cuja cadeia é igual à do lipídio DMPC, mas com cabeça polar diferente, que dissocia em água. A presença de cargas introduz algumas modificações das propriedades térmicas da solução de lipídios, como pode ser verificado na figura 1.5b para o calor específico da dispersão de vesículas do lipídio carregado DMPG: ocorrem um alargamento do pico e o surgimento de novos picos. A dissociação da cabeça polar introduz uma força repulsiva entre os lipídios, desfavorecendo a fase gel. Este efeito diminui e desaparece com a adição de sal. As figuras 1.5a e 1.5c ilustram este efeito. Na figura 1.5a são comparadas as curvas de calor específicodo DMPG para várias concentrações de sal. Vemos o alargamento do pico desaparecer com a adição de sal. Em alta concentração de sal vemos um único pico, como 


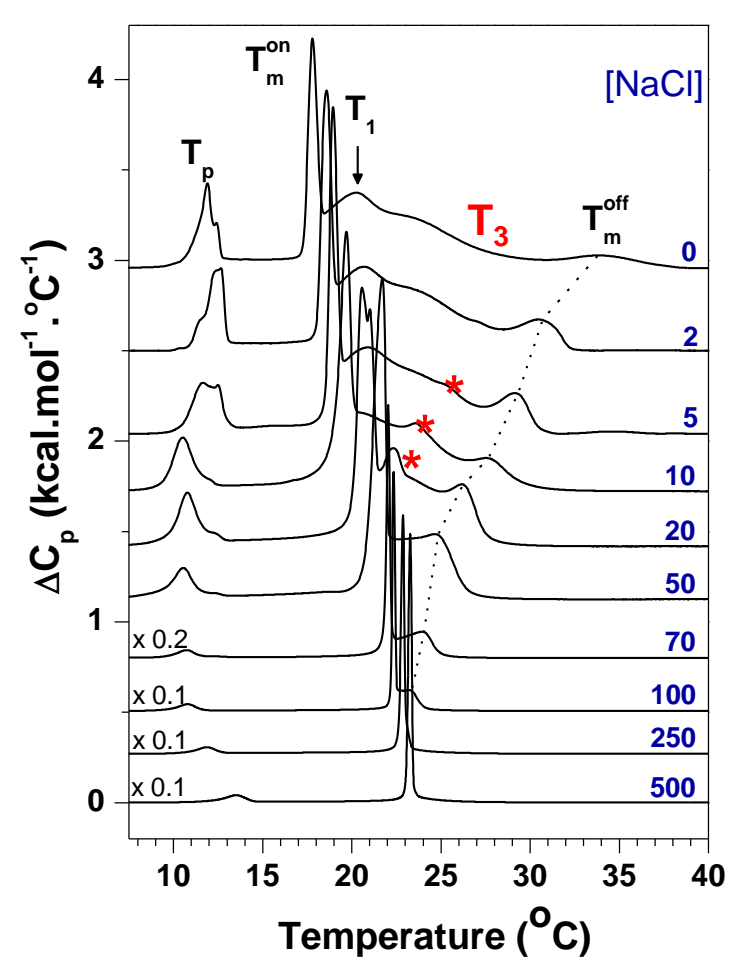

(a)

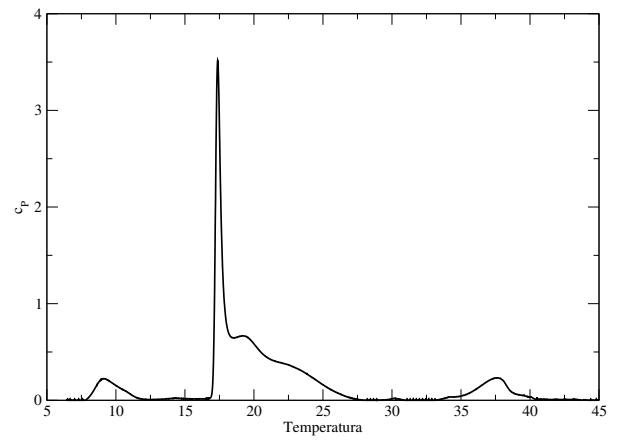

(b)

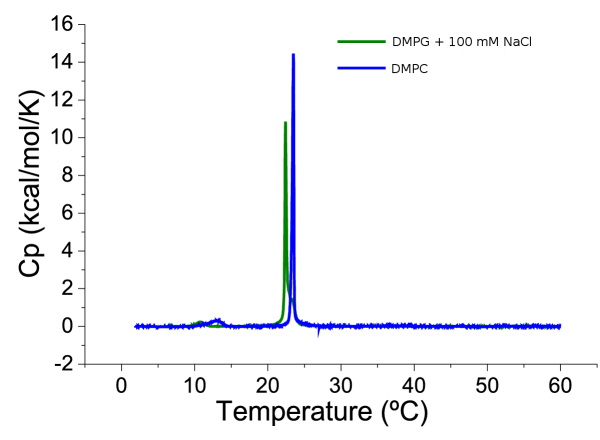

(c)

Figura 1.5: Curvas de calor específico em função da temperatura para: (a) solução de vesículas de DMPG (difosfatidildimiristoil glicerol) para diferentes concentração de sal [9]; (b) solução de vesículas de DMPC sem sal na solução; (c) vesículas construídas com DMPC e DMPG com 100mM de sal. O pico estreito da transição ordem-desordem das cadeias é substituído por um pico alargado para o lipídio ionizável DMPG. O alargamento desaparece com a adição de sal em alta concentração. (b) e (c) representam medidas efetuadas no Laboratório de Biofísica do IFUSP.

no caso do lipídio neutro. Na figura 1.5c, são comparadas as curvas de calor específico de soluções dos dois lipídios, com o mesmo tamanho de cadeia hidrocarbônica, mas cabeças polares distintas. O DMPC é um lipídio com cabeça polar, neutra, e o DMPG é um lipídio com cabeça polar ionizável, que se torna carregada em solução. No caso em que é adicionada uma grande quantidade de sal à solução de lipídios ionizáveis, as posições dos dois picos do calor específico coincidem, e parece razoável supor que a alta concentração 


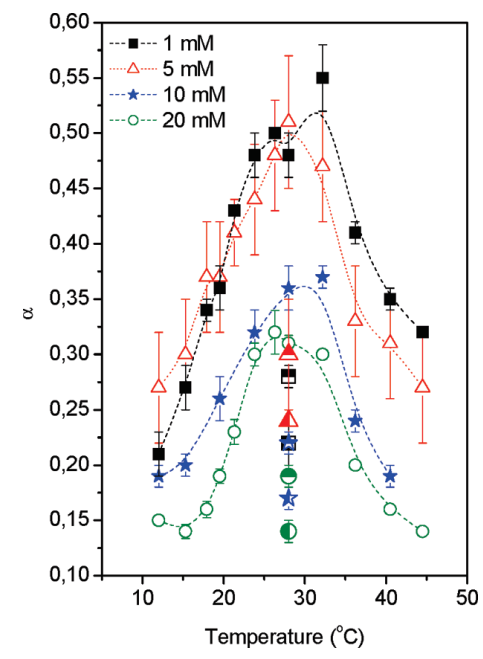

(a) ionização da vesícula

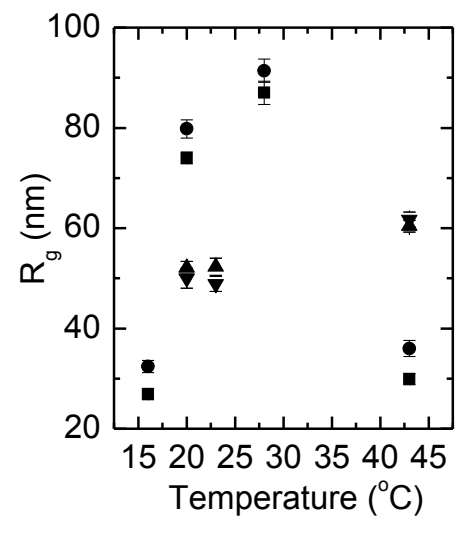

(b) raio de giração da vesícula

Figura 1.6: (a) Ionização da vesícula carregada em função da temperatura, ao longo da região de transição, para diferentes concentrações da solução iônica. Interpretação de dados de condutividade e eletroforese. Figura 12 de [10] (b) Raio de giração da vesícula em função da temperatura, obtido a partir de medidas de espalhamento de luz estático. Figura de [6]

de sal blinda o campo elétrico devido as cabeças de DMPG carregadas e, dessa forma, o DMPG se comporta de forma semelhante ao DMPC, ficando a dinâmica do sistema dominada pela interação entre as cadeias.

A solução de lipídios ionizáveis apresenta outras propriedades especiais, em comparação com os lipídios neutros. A região de alargamento do calor específico é acompanhada de um aumento da carga superficial da vesícula (figura 1.6a) e de um crescimento do raio de giração (figura 1.6b) que não pode ser explicado em termos do aumento da área média por lipídio associada à transição ordem - desordem das cadeias.

Não há ainda uma descrição microscópica bem estabelecida do efeito da carga sobre a transição ordem - desordem das cadeias lipídicas no caso de cabeça polar ionizável, apesar de várias tentativas [11, 12]. Nosso objetivo 
é o estudo de modelos estatísticos para a transição ordem - desordem das cadeias de uma bicamada lipídica na presença de dissociação das cabeças polares em solução aquosa.

Do ponto de vista teórico, foram propostos dois modelos estatísticos básicos para descrever a transição gel - fluido. Na década de 1970, J. Nagle [13], desenvolveu um modelo que focalizava a entropia das cadeias lipídicas para a qual podiam ser obtidos resultados analíticos. A interação entre os lipídios era adicionada em um abordagem de campo médio. Este modelo acabou sendo abandonado em favor de um modelo mais simples, proposto por Doniach [14], em que os graus de liberdade das cadeias são representados em termos de dois estados de lipídios.

\section{Modelo de Doniach}

O modelo de Doniach [14], de 1978, descreve todos os possíveis estados das cadeias dos lipídios formadores da membrana simplesmente por dois estados, representados na figura 1.7. Na membrana, a configuração de menor energia das cadeias, denominada all-trans, é única e consiste em uma par de cadeias "rígidas", ocupando o mínimo de espaço na superfície da membrana. Os estados de cadeias desordenadas são multipolos e possuem maior energia. No modelo de Doniach, as várias configurações desordenadas são representadas por um único estado, ou seja, as configurações de cadeias desordenadas são descritas por um único estado com grande degenerescência. 


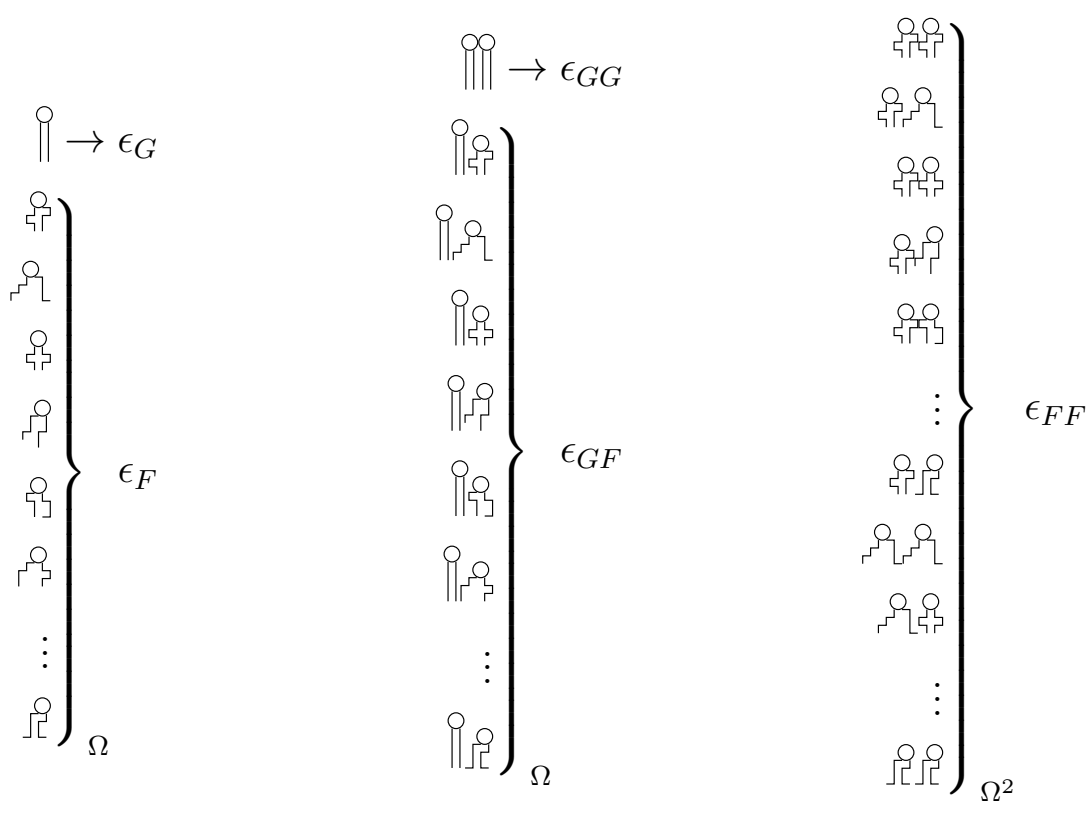

Figura 1.7: Representação simplificada dos estados das caudas lipídicas do modelo de Doniach

Dessa forma, a função de partição de uma cadeia isolada seria

$$
z_{\text {cadeia }}=\mathrm{e}^{-\frac{E_{G}}{k_{B} T}}+\Omega \mathrm{e}^{-\frac{E_{F}}{k_{B} T}}
$$

onde $E_{G}$ e $E_{F}$ são as energias do estado gel e fluido, respectivamente, $k_{B}$ é a constante de Boltzmann, $T$ a temperatura absoluta e $\Omega$ é a degenerescência, que representa a quantidade de possíveis estados desordenados diferentes. Esta quantidade pode ser obtida ajustando-se dados experimentais, ou pode ser estimada considerando-se o comprimento das cadeias e possíveis dobras.

Doniach assume a superfície da membrana como um plano infinito, e o efeito da água é representado indiretamente através da interação entre lipídios e a interação entre as camadas da bicamada é ignorada. Além disso, também argumenta que a energia de interação entre as cadeias dos lipídios é bem 
representada por uma atração de curto alcance, como van der Waals, e uma repulsão devido à competição do espaço ocupável pela cadeia com as cadeias dos lipídios vizinhos. Assim, as interações relevantes incluem apenas lipídios vizinhos.

Nesse modelo, o único grau de liberdade do lipídio está na conformação de suas cadeias de carbono, não há representação da mobilidade típica da fase fluida. Pelos argumentos do próprio Doniach, isto é justificável, pois a entropia dessa mobilidade é tipicamente da ordem de $0.5 k_{B}$ por molécula, e é pequena se a compararmos com a diferença de entropia entre o estado gel e o estado fluido, que é da ordem de $14 k_{B}$ por molécula.

O termo de competição pelo espaço ocupável é complicado de estimar. Doniach usa uma fórmula atribuída a Marcelja [15], onde uma espécie de pressão lateral média $\Pi$ dá origem a uma diferença de potencial termodinâmico $\Pi \Delta A$, onde $\Delta A$ é a mudança da área ocupada pela cadeia, quando o lipídio no estado gel vai para o estado fluido. Ou seja, os sítios no estado gel contribuem com um termo $\prod a_{G}$ para a energia total e os sítios no estado fluido com $\Pi a_{F}$, onde $a_{G}$ e $a_{F}$ são as áreas ocupadas pelos lipídios nos estados ordenado e desordenado, respectivamente.

O modelo é um modelo de dois estados na presença de um campo que vem de duas contribuições: a pressão lateral e a degenerescência. Doniach mapeia seu modelo no modelo de Ising bidimensional, com campo dependente da temperatura, que apresenta coexistência entre estados de "magnetização" positiva (gel) e negativa (fluida) no plano pressão temperatura. 


\begin{tabular}{c|c|c|c|c} 
Estado & $m$ & $A_{m}\left[\AA^{2}\right]$ & $E_{m}\left[10^{-13} \mathrm{erg}\right]$ & $D_{m}$ \\
\hline GEL & 1 & 20,4 & 0 & 1 \\
& 2 & 21,86 & 0,45 & 4 \\
& 3 & 23,54 & 0,45 & 4 \\
& 4 & 25,5 & 0,45 & 4 \\
& 5 & 21,86 & 0,9 & 20 \\
& 6 & 23,54 & 0,9 & 16 \\
& 7 & 25,5 & 0,9 & 12 \\
& 8 & 23,54 & 1,35 & 64 \\
FLUIDO & 9 & 25,5 & 1,35 & 96 \\
& 10 & 34,0 & 2,78 & 354294
\end{tabular}

Tabela 1.1: Tabela das áreas $A_{m}$, energias $E_{m}$ e multiplicidade $D_{m}$ dos dez estados $m$ do modelo de dez estados. Reprodução da tabela 1 da referência [17].

\section{Modelo de Mouritsen}

Em 1980, Pink e Chapman [16] introduzem um modelo de rede para bicamadas com proteínas, com dez estados. Baseado no modelo de Pink e Chapman para membrana com proteínas, Mouritsen, Pink, Zuckermann e colaboradores publicaram em 1983 um artigo[17] onde apresentam um modelo de dois estados e um modelo de dez estados.

Assim como no modelo de Doniach, a camada lipídica é representada por uma rede triangular bidimensional. Tanto no modelo de dois estados, como no modelo de dez estados, a energia de interação entre pares de cadeias nos estados gel - gel, gel - fluido, e fluido - fluido são computadas. Além disso, é atribuída uma energia para cada estado.

A grande diferença entre o modelo de dois estados e o modelo de dez estados é a existência de 8 estados intermediários entre o estado gel e o estado fluido. Estes estados são chamados de estados "dobrados" e, para cada um desses estados, é preciso definir a área ocupada pelas cadeias, a 
multiplicidade do estado, a energia de interação entre estados e a energia do estado. Na tabela 1.1 reproduzimos um exemplo de alguns desses valores. Particularmente, acreditamos ser mais apropriado interpretar que no modelo de dez estados, todos os $\Omega$ estados fluido são distribuídos em 9 estados fluidos distintos.

Variantes desses modelos têm sido amplamente utilizadas na literatura como modelo base para bicamadas lipídicas neutras, também no caso de misturas, de presença de colesterol, ou no estudo de permeabilidade [9, 18, $19,20,21,22,23,24]$.

Neste trabalho, iremos nos concentrar no modelo de dois estados, pois é mais simples que o modelo de dez estados, e possui os elementos mínimos necessários para o aparecimento da transição de fase.

\section{Nossos modelos}

Fomos motivados pelos estudos experimentais de soluções de vesículas de DMPG realizados no laboratório de biofísica do IFUSP [4], nos quais mostrouse útil comparar as membranas construídas com DMPG (difosfatidildimiristoil glicerol) com as construídas com DMPC (difosfatidildimiristoil colina). Estes fosfolipídios diferem apenas na composição da cabeça polar. A cabeça polar do DMPG, fig. 1.8a, se dissocia, ou seja, perde um íon de sódio para a solução, tornando-se carregada. Já a cabeça polar do DMPC, fig. 1.8b, não se dissocia e, além disso, possui dipolo elétrico.

Apesar de não ser possível inferir a física microscópica do sistema a partir das propriedades termodinâmicas, as mesmas sugerem hipóteses sobre a estrutura e dinâmica do sistema. Neste trabalho estamos propondo um modelo 
simplificado para a transição gel - fluido de vesículas construídas com lipídios ionizáveis.

Visto que a dissociação, bem como sua dependência das condições termodinâmicas, tais como a temperatura e a força iônica, parecem ser essênciais para entender o fenômeno da transição de membranas carregadas, vamos acoplar um modelo de membrana a um modelo de solução iônica.

Propomos uma modificação do modelo de Doniach, que inclue flutuação de densidade, considerando os estados de Doniach em um gás de rede. Dessa forma, definimos distância entre os lipídios, o que nos permite descrever interações eletrostáticas entre os mesmos.

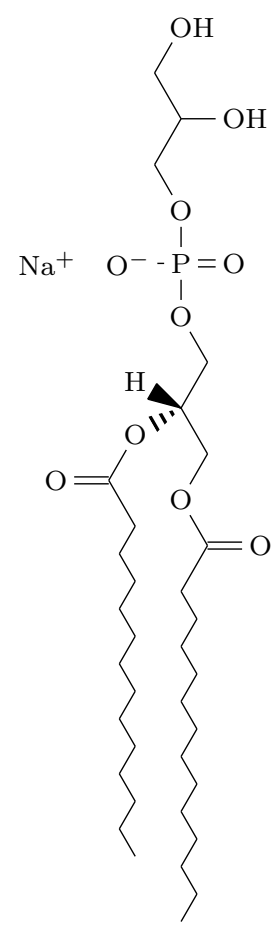

(a) DMPG

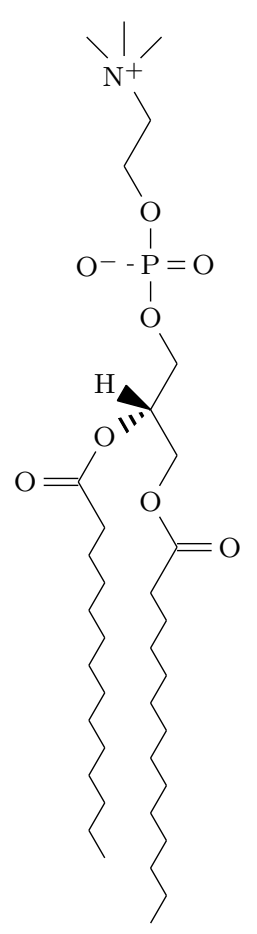

(b) DMPC

Figura 1.8: Estrutura esquemática dos fosfolipídios DMPC, com cabeça dipolar (a), e DMPG, com cabeça ionizável (b). As caudas hidrocarbônicas dos dois lipídeos são idênticas.

Numa segunda etapa, adaptamos o modelo de gás de rede de Doniach, adequado para vesículas construídas com lipídios neutros, que não se dissociam em água, para bicamadas construídas com lipídios que se dissociam em água. No modelo de Doniach e no nosso modelo de gás de rede para os lipídios a única interação entre os lipídios é a interação atrativa, de natureza hidrofóbica. Propomos incluir as interações eletrostáticas introduzindo uma 


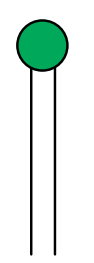

(a)

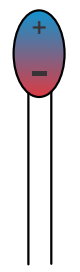

(b)

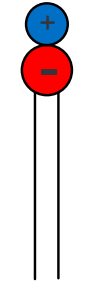

(c)

Figura 1.9: Diferentes representações de lipídio: (a) lipídio neutro simplificado; (b) lipídio neutro com dipolo elétrico na cabeça polar; (c) lipídio ionizável.

estrutura da cabeça polar lipídica, como na figura 1.9. Nos modelos neutros, a cabela polar não desempenha papel relevante e as cadeias hidrocarbônicas são representadas através dos dois estado de lipídio: ordenado ou desordenado (figura 1.9a). Vamos adicionar ao modelo neutro o papel das cabeças polares, incluindo cargas explicitamente, seja na forma de dipolo(fig. 1.9b), seja na forma de um par ionizável (fig. 1.9c). Quanto à solução iônica, escolhemos, por simplicidade, a versão na rede do modelo primitivo restrito $[25]$.

No capítulo 2, efetuamos uma revisão do modelo de Doniach e introduzimos os três modelos para membrana analisados nesta tese, bem como o modelo para solução iônica (LRPM). No capítulo 3, analisamos as propriedades do modelo de gás de rede de Doniach, que denominamos DLG, na abordagem de campo médio. No capítulo 4, as propriedades do modelo DLG são obtidas através de simulações numéricas. O modelo LRPM é definido no capítulo 5, no qual é efetuado um estudo sistemático das simulações de sistemas carregados. No capítulo 6, definimos os modelos com representação de cargas na cabeça lipídica e analisamos algumas de suas propriedades através de simulações numéricas. No capítulo 7, apresentamos algumas conclusões e 
considerações finais. 


\section{Capítulo 2}

\section{Modelos Estatísticos}

Neste capítulo, vamos introduzir os modelos investigados neste trabalho. Em

primeiro lugar, fazemos uma revisão do modelo de Doniach para introduzir as ideias e fixar a notação. Dada a ausência da noção de distância, necessária para o tratamento da interação Coulombiana, apresentamos uma versão gás de rede para os estados de Doniach. Por outro lado, para representar lipídios que dissociam, introduzimos graus de liberdade adicionais, que representam as cargas da cabeça polar lipídica. Finalmente, propomos a representação da solução aquosa de lipídios carregados através do acoplamento do modelo de membrana carregada ao modelo primitivo restrito na rede (LRPM).

\subsection{Modelo de Doniach}

O modelo é definido em um reticulado, sendo que o estado de cada sítio partícula $i$ é descrito pela variável $\eta_{i}$, que assume os valores 0 e 1 , representando respectivamente o estado fluido e o estado gel das cadeias hidro- 


$$
\begin{array}{lll}
\eta=1 & 1 \\
& \text { (a) Ordenado } \\
& & \\
\text { ९ } & \eta=0 & \Omega
\end{array}
$$

(b) Desordenado

Figura 2.1: Representações pictóricas do estado do lipídio, associação com a descrição no modelo de Doniach e sua multiplicidade, para lipídios no estado ordenado(a) e desordenado(b)

carbônicas do lipídio formador da membrana.

A energia total de um estado microscópico do sistema é composta pela soma das energias de interação entre as partículas, sendo que neste modelo, as interações se restringem aos primeiros vizinhos. A energia de interação entre os sítios no estado gel é representada por $\epsilon_{G}$, entre sítios no estado fluido por $\epsilon_{F}$ e a interação entre sítios no estado gel e os sítios no estado fluido por $\epsilon_{G F}$. Ver figura 1.7. Dessa forma, temos que a energia total do sistema é dada por

$E=-\epsilon_{G} \sum_{(i j)} \eta_{i} \eta_{j}-\epsilon_{F} \sum_{(i j)}\left(1-\eta_{i}\right)\left(1-\eta_{j}\right)-\epsilon_{G F} \sum_{(i j)}\left[\eta_{i}\left(1-\eta_{j}\right)+\left(1-\eta_{i}\right) \eta_{j}\right]$

onde $(i j)$ indica que a soma é realizada sobre os $\frac{q}{2}$ primeiros vizinhos. O reticulado que contém o sistema se restringe a definir os vizinhos de cada sítio.

A noção de área ocupada pelo sistema vem da soma das áreas ocupadas por cada lipídio nos dois possíveis estados, ou seja, $a_{G}$ para os lipídios no estado gel e $a_{F}$ para os lipídios no estado fluido. Este elemento contribui 
com o termo $\Pi A$ na energia total

$$
A=a_{G} \sum_{i} \eta_{i}+a_{F} \sum_{i}\left(1-\eta_{i}\right)
$$

sendo que a área total $A$ é composta da soma das áreas das cabeças de todos os lipídios e П é uma espécie de pressão lateral.

A equação 2.1 pode ser reescrita em termos de variáveis de spin com a transformação

$$
\eta_{i}=\frac{1+\sigma_{i}}{2}
$$

para

$$
\begin{aligned}
& \eta_{i}=1 \quad \rightarrow \quad \sigma_{i}=+1 \\
& \eta_{i}=0 \quad \rightarrow \quad \sigma_{i}=-1 .
\end{aligned}
$$

A energia 2.1, fica

$$
E=\frac{1}{4}\left(-\epsilon_{F}-\epsilon_{G}+2 \epsilon_{G F}\right) \sum_{(i j)} \sigma_{i} \sigma_{j}+\frac{1}{4}\left(\epsilon_{F}-\epsilon_{G}\right) \sum_{(i j)}\left(\sigma_{i}+\sigma_{j}\right)
$$

e os termos de área e de degenerescência contribuem para o expoente da função de partição no ensemble das pressões com a expressão

$$
\left[\frac{\Pi}{2}\left(a_{G}-a_{F}\right)-\frac{k_{B} T}{2} \ln \Omega\right] \sum_{i} \sigma_{i}
$$

Obtém-se uma Hamiltoniana efetiva de Ising na presença de campo magnético 


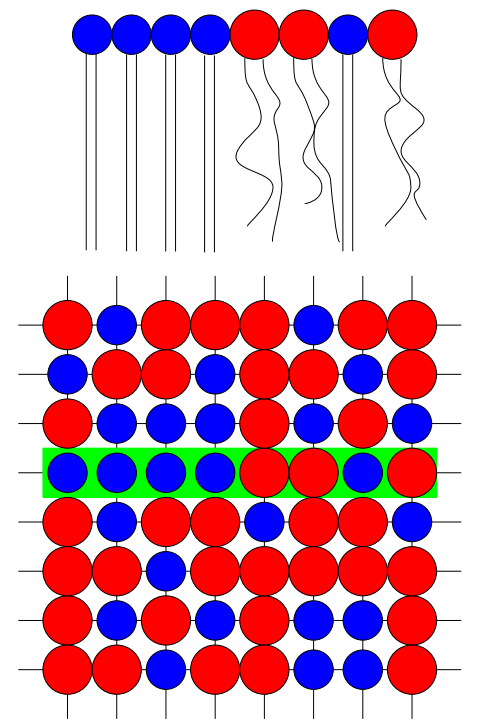

(a)
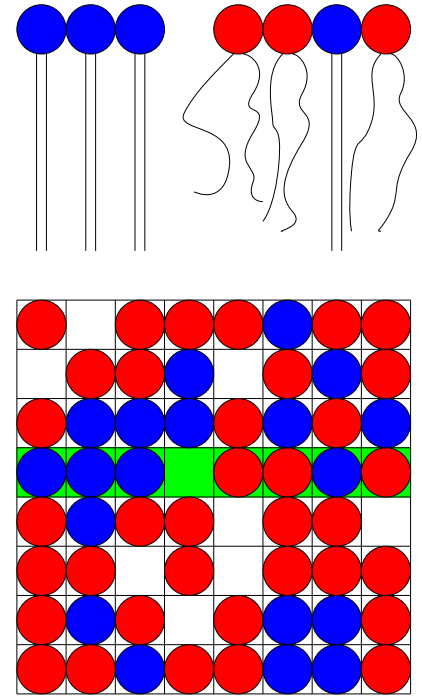

(b)

Figura 2.2: Corte lateral da membrana (parte superior) e visão do modelo bidimensional (parte inferior). (a) Representação de um estado qualquer de uma membrana na visão do modelo de Doniach. (b) Representação de estado no espírito de gás de rede de Doniach (DLG).

representado na expressão anterior 2.7. A pressões suficientemente baixas, a transição entre os estados ordenado (gel) e desordenado (fluido) é de primeira ordem.

\subsection{Introduzindo flutuações de densidade: gás de rede de Doniach (DLG)}

Não ter distância definida impossibilita a inserção de interações Coulombianas que são dependentes da distância. Para introduzir a distância, vamos reinterpretar o modelo de dois estados de Doniach e transformá-lo em um modelo de gás de rede.

Faremos isso substituindo a noção de área dos modelos de Doniach et alii 
pela noção de área por partícula dos modelos de gás de rede. No gás de rede, o volume ocupado médio por partícula é função da distância média entre as partículas. Analogamente, a área ocupada média por lipídio será função da distância média entre lipídios, independente do estado do lipídio.

Vamos introduzir sítios que correspondem a espaços vazios e definir que a interação de qualquer sítio com um sítio vazio é nula. Sendo assim, a equação 2.1 é reescrita como

$E=\sum_{(i j)} \delta_{i} \delta_{j}\left(-\epsilon_{G} \eta_{i} \eta_{j}-\epsilon_{F}\left(1-\eta_{i}\right)\left(1-\eta_{j}\right)-\epsilon_{G F}\left[\eta_{i}\left(1-\eta_{j}\right)+\left(1-\eta_{i}\right) \eta_{j}\right]\right)$,

onde $\delta_{i}$ assume o valor 1 para o sítio ocupado e 0 para o sítio vazio.

Os modelos de Doniach et alii são definido no ensemble das pressões. Com estas mudanças, a pressão lateral $\Pi$, termodinamicamente conjugada à área, deixou de ser um parâmetro interessante para os cálculos do modelo. O novo modelo é descrito no ensemble grande canônico, tendo o potencial químico e a temperatura como grandezas constantes.

É conveniente descrever as variáveis $\eta$ e $\delta$ por uma única variável. Para isso, vamos mapear as variáveis $\eta$ e $\delta$ na variável $\sigma$, da seguinte forma

$$
\begin{aligned}
& \eta_{i}=1 \quad \rightarrow \quad \sigma_{i}=+1 \\
& \eta_{i}=0 \quad \rightarrow \quad \sigma_{i}=-1 \\
& \delta_{i}=0 \quad \rightarrow \quad \sigma_{i}^{2}=0 \\
& \delta_{i}=1 \quad \rightarrow \quad \sigma_{i}^{2}=1
\end{aligned}
$$


pelas relações

$$
\begin{aligned}
& \eta_{i}=\frac{1+\sigma_{i}}{2}, \\
& \delta_{i}=\sigma_{i}^{2} .
\end{aligned}
$$

A energia do estado do sistema 2.8, descrito em função de $\sigma$, fica

$$
\begin{aligned}
E= & \frac{1}{4}\left(-\epsilon_{F}-\epsilon_{G}+2 \epsilon_{G F}\right) \sum_{(i j)} \sigma_{i} \sigma_{j}+\frac{1}{4}\left(\epsilon_{F}-\epsilon_{G}\right) \sum_{(i j)} \sigma_{i} \sigma_{j}\left(\sigma_{i}+\sigma_{j}\right) \\
& +\frac{1}{4}\left(-\epsilon_{F}-\epsilon_{G}-2 \epsilon_{G F}\right) \sum_{(i j)} \sigma_{i}^{2} \sigma_{j}^{2}
\end{aligned}
$$

de onde definimos novos parâmetros em função dos parâmetros originais

$$
\begin{gathered}
J=\frac{\epsilon_{G}+\epsilon_{F}-2 \epsilon_{G F}}{4} \\
\Delta=\frac{1}{4}\left(\epsilon_{G}-\epsilon_{F}\right) \\
K=\frac{\epsilon_{G}+\epsilon_{F}+2 \epsilon_{G F}}{4} .
\end{gathered}
$$

Após a mudança de variável, a energia do sistema, equação 2.15, corresponde à energia do modelo Blume - Emery - Griffiths [26], acrescida de um termo de acoplamento cúbico, $\sigma_{i} \sigma_{j}^{2}$.

A energia do microestado $\{\sigma\}$, para estes novos parâmetros, é dada por

$$
E(\{\sigma\})=-J \sum_{(i j)} \sigma_{i} \sigma_{j}-\Delta \sum_{(i j)} \sigma_{i} \sigma_{j}\left(\sigma_{i}+\sigma_{j}\right)-K \sum_{(i j)} \sigma_{i}^{2} \sigma_{j}^{2}
$$


e a grande função de partição é dada por

$$
\Xi(T, \mu)=\sum_{\{\sigma\}} \Omega^{N_{F}} \exp \left\{-\beta\left(E(\{\sigma\})-\mu \sum_{i} \sigma_{i}^{2}\right)\right\}
$$

onde $N_{F}$ é a quantidade de lipídios no estado fluido, desordenado. Lembrando que $\sum_{\{\sigma\}}$ simboliza a soma sobre todos os estados microscópicos $\{\sigma\}=$ $\left\{\sigma_{1}, \sigma_{2}, \ldots, \sigma_{A}\right\}$, onde $A$ é a quantidade de sítios e a área total do sistema. Isto equivale em uma soma em $A$ dimensões sobre os estados de cada sítio,

$$
\sum_{\{\sigma\}}=\sum_{\sigma_{1}} \sum_{\sigma_{2}} \cdots \sum_{\sigma_{A}}
$$

Estamos interessados na transição ordem - desordem das cadeias lipídicas. Ela é caracterizada pelo aumento abrupto da área da superfície da membrana, com as caudas dos lipídios passando do estado ordenado para o estado desordenado. Nos modelos de Doniach et alii estes dois parâmetros são indistinguíveis. Neste novo modelo, este dois parâmetros são a priori independentes e estão relacionados através da Hamiltoniana.

Tomaremos a liberdade de atribuir ao parâmetro que descreve o estado das caudas dos lipídios o nome de "magnetização", definida como

$$
M=\sum_{i} \sigma_{i}
$$

com $M / A=1$ e $M / A=-1$ nos estados completamente ordenado e desordenado, respectivamente.

Vamos assumir que a quantidade de lipídios que forma a membrana não se 
altera. Esta hipótese está baseada em um estudo experimental para vesículas lipídicas, que mostra que mesmo sob variação de tamanho, o agregado se mantém inteiro [4, 27]. A área superficial da membrana dividida pela quantidade de lipídios, ou seja, a área ocupada por lipídio, descreve o sistema tão bem quanto a área superficial total. Nos nossos modelos, estamos olhando para uma região fixa da superfície da camada lipídica, de área $A$. A quantidade de lipídios de uma determinada configuração é dada por

$$
N=\sum_{i} \sigma_{i}^{2}
$$

e, portanto, a densidade é a quantidade de lipídios dividida pela quantidade total de sítios e a área ocupada por lipídio é o inverso da densidade.

\subsection{Modelo para a solução iônica: modelo primitivo restrito na rede $(\mathrm{LRPM})$}

O modelo primitivo restrito é um modelo de esferas rígidas carregadas. A versão na rede, o modelo primitivo restrito na rede, é um gás de rede de partículas carregadas. Este sistema representa íons em solução, onde a solução é representada pelos sítios sem partículas.

O modelo é definido em uma rede cúbica, na qual as interações são dadas pela restrição de volume excluído fornecido pela rede e pelo potencial Coulombiano,

$$
\phi_{i j}=\frac{q_{i} q_{j}}{r_{i j}},
$$


onde $\mathbf{r}_{i}$ é o vetor posição do íon $i, r_{i j}=\left|\mathbf{r}_{i}-\mathbf{r}_{j}\right|$ é a distância entre os íons $i$ e $j$ e a carga da partícula/íon $i$ é descrita por $q_{i}=+1$ ou -1 . As partículas estão restritas à caixa de simulação a fim de conservar a densidade do sistema.

O modelo apresenta um comportamento rico, mas em nosso estudo, consideraremos apenas soluções de baixa densidade.

\subsection{Membranas com cargas: DLG com car- gas (CDLG)}

Os modelos de Doniach e suas variantes, bem como o nosso modelo DLG, não consideram explicitamente a distribuição de carga do lipídio neutro. A distribuição de carga é essencialmente a distribuição de carga na cabeça polar, que é bem representada por um dipolo, como na figura 1.9b.

Inspirados pelo modelo LRPM, vamos representar a cabeça polar do lipídio como um par neutro de sítios carregados, como na figura 1.9c. Então, definimos que o lipídio dissociado é aquele cujo o contraíon positivo não pertence à sua vizinhança imediata.

No modelo DLG, sem carga, os sítios nos estado gel $(\sigma=1)$ e fluido $(\sigma=-1)$ representam lipídios, respectivamente, ordenados e desordenado, e sítios com $\sigma=0$ são espaços vazios. A primeira mudança a ser feita será passar a representar o lipídio por 2 sítios. O sítio inferior passa a representar simultaneamente o estado da cauda e a parte negativa da cabeça polar, e será identificado por $\sigma \neq 0$ e $q=-1$. O sítio superior, de carga positiva, será identificados por $\sigma=0$ e $q=1$. Em outras palavras, substituimos a 
Figura 2.3: Modelo simplificado do conjunto membrana ionizável e solução salina para o estado fluido.

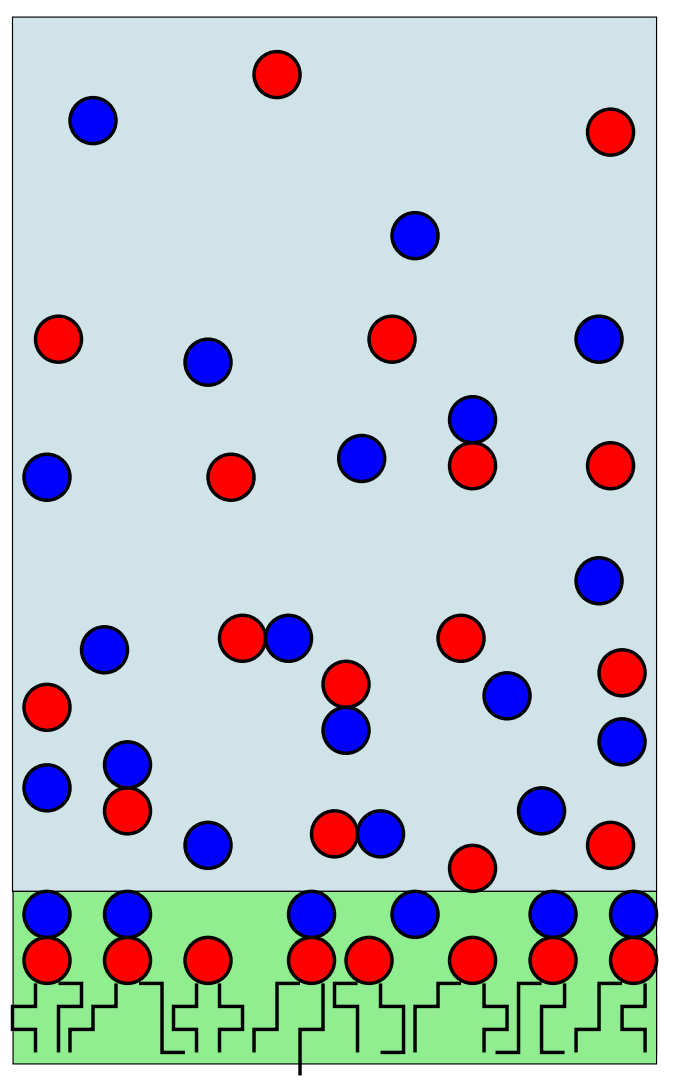

representação da figura 1.9a pela da figura 1.9c. Na figura 2.3, apresentamos um esquema do modelo da membrana com carga. 


\section{Capítulo 3}

\section{Modelo DLG: abordagem de}

\section{Campo Médio}

Neste capítulo estudaremos através da abordagem de campo médio a versão gás de rede do modelo de Doniach, o modelo DLG. O modelo de Doniach foi feito para descrever minimamente a transição ordem - desordem, ou gel - fluido, de bicamadas lipídicas. A versão gás de rede introduz flutuação de densidade ao modelo de Doniach, descrevendo a área ocupada média por lipídio de maneira um pouco mais realista. Graças a isso, pela abordagem de campo médio, o modelo descreve qualitativamente tanto a transição gel fluido de bicamadas lipídicas como a transição líquido condensado - líquido expandido e a transição líquido expandido - gás de monocamadas de Langmuir.

Vamos apresentar diagramas de fase $t-\mu$, onde para um intervalo de parâmetros, observa - se a presença de um ponto triplo e de uma região reentrante. Apresentaremos também um desses diagramas reescritos em termos 
da pressão lateral e da temperatura.

\subsection{Aproximação de Campo Médio}

A abordagem de campo médio adotada consiste em aproximar as interações entre primeiros vizinhos pela interação entre todas as $N$ partículas do sistema, conhecida por Curie - Weiss. A grande função de partição 2.20 é reescrita na forma

$$
\Xi(T, \mu, H)=\sum_{\{\sigma\}} \Omega^{N_{F}} \exp \{-\beta \tilde{\phi}\},
$$

com

$$
\begin{aligned}
\tilde{\phi}(\{\sigma\})= & -\tilde{J} \sum_{i} \sigma_{i} \sum_{j} \sigma_{j}-\tilde{\Delta}\left(\sum_{i} \sigma_{i} \sum_{j} \sigma_{j}^{2}+\sum_{i} \sigma_{i}^{2} \sum_{j} \sigma_{j}\right) \\
& -\tilde{K} \sum_{i} \sigma_{i}^{2} \sum_{j} \sigma_{j}^{2}-\mu \sum_{i} \sigma_{i}^{2}-H \sum_{i} \sigma_{i}
\end{aligned}
$$

onde as novas variáveis $\tilde{J}, \tilde{\Delta}$ e $\tilde{K}$ devem ser tais que a energia na aproximação de campo médio seja igual à energia original em algum estado de referência conhecido. Foi escolhido o estado fundamental com todos os sítios no estado ordenado $\sigma=1$. Portanto,

$$
E(T=0)=E_{C M}(T=0)
$$


que implica na relação

$$
-J A \frac{q}{2}-2 \Delta A \frac{q}{2}-K A \frac{q}{2}=-\tilde{J} A^{2}-2 \tilde{\Delta} A^{2}-\tilde{K} A^{2}
$$

Para este sistema de sítios de área unitária, a quantidade total de sítios, $A$, é identificada como a área do sistema. A relação entre os parâmetros originais e os novos é dada por:

$$
\tilde{J}=\frac{q}{2 A} J \quad ; \quad \tilde{\Delta}=\frac{q}{2 A} \Delta \quad \text { e } \quad \tilde{K}=\frac{q}{2 A} K \quad
$$

onde $q$ é o número de coordenação da rede, em outra palavras, a quantidade de sítios que são primeiros vizinhos de um sítio qualquer. Neste trabalho, utilizaremos a rede quadrada, e portanto, $q=4$.

Vamos definir variáveis auxiliares $M=M\left(\sigma_{1}, \sigma_{2}, \ldots, \sigma_{A}\right)$ e $Q=Q\left(\sigma_{1}^{2}, \sigma_{2}^{2}, \ldots, \sigma_{A}^{2}\right)$, de maneira tal que a grande função de partição possa ser expressa na forma $\Xi(T, \mu, H)=\sum_{\{\sigma\}} \Omega^{N_{F}} \exp \left\{-\beta\left(-\tilde{J} M^{2}-2 \tilde{\Delta} M Q-\tilde{K} Q^{2}-\mu Q-H M\right)\right\}$

É possível desacoplar os termos cruzados $Q M$ pelo método de completar quadrados,

$$
\Xi(T, \mu, H)=\sum_{\{\sigma\}} \Omega^{N_{F}} \mathrm{e}^{\left.\beta \tilde{J}\left(M+\frac{\tilde{\tilde{J}}}{\tilde{J}} Q\right)^{2}+\beta\left(-\frac{\tilde{\tilde{\tilde{U}}}^{2}}{\tilde{J}}+\tilde{K}\right) Q^{2}+\beta \mu Q+\beta H M\right)}
$$

e, com a identidade gaussiana,

$$
e^{a^{2}}=\frac{1}{\sqrt{\pi}} \int_{-\infty}^{\infty} \mathrm{e}^{-x^{2}+2 a x} \mathrm{~d} x
$$


os termos quadráticos são linearizados,

$$
\begin{aligned}
\Xi(T, \mu, H)= & \sum_{\{\sigma\}} \Omega^{N_{F}} \int_{-\infty}^{\infty} \int_{-\infty}^{\infty} \mathrm{e}^{-x^{2}+2 \sqrt{\beta \tilde{J}}\left(M+\frac{\Delta}{J} Q\right) x-y^{2}+2 \sqrt{\beta\left(-\frac{\tilde{\Delta}^{2}}{\tilde{J}}+\tilde{K}\right)} Q y} \\
& \mathrm{e}^{+\beta \mu Q+\beta H M} \mathrm{~d} x \mathrm{~d} y
\end{aligned}
$$

Reordenando os termos da exponencial e invertendo a ordem das somatórias e integrais, obtemos,

$$
\begin{aligned}
\Xi(T, \mu, H) & =\int_{-\infty}^{\infty} \int_{-\infty}^{\infty} \mathrm{e}^{-x^{2}-y^{2}} \\
& \sum_{\{\sigma\}} \Omega^{N_{F}} \mathrm{e}^{+(2 \sqrt{\beta \tilde{J}} x+\beta H) M+\left(2 \sqrt{\beta\left(\frac{-\tilde{\tilde{\alpha}}^{2}}{\tilde{J}}+\tilde{K}\right)} y+2 \sqrt{\beta \tilde{J}} \frac{\Delta}{J} x+\beta \mu\right) Q} \mathrm{~d} x \mathrm{~d} y .
\end{aligned}
$$

$\mathrm{Na}$ esperança de ser prático, serão definidas novas variáveis

$$
\begin{gathered}
\alpha_{1}=2 \sqrt{\beta \tilde{J}}, \quad \alpha_{2}=\beta H, \quad \alpha_{3}=2 \sqrt{\beta\left(-\frac{\tilde{\Delta}^{2}}{\tilde{J}}+\tilde{K}\right)}, \\
\alpha_{4}=2 \sqrt{\beta \tilde{J}} \frac{\Delta}{J} \quad \text { e } \quad \alpha_{5}=\beta \mu
\end{gathered}
$$

tal que a grande função de partição nestas novas variáveis é escrita como

$$
\Xi(T, \mu, H)=\int_{-\infty}^{\infty} \int_{-\infty}^{\infty} \mathrm{e}^{-x^{2}-y^{2}} \sum_{\{\sigma\}} \Omega^{N_{F}} \mathrm{e}^{+\left(\alpha_{1} x+\alpha_{2}\right) M+\left(\alpha_{3} y+\alpha_{4} x+\alpha_{5}\right) Q} \mathrm{~d} x \mathrm{~d} y
$$

Como os termos $M$ e $Q$ estão desacoplados, a somatória em 3.13 pode ser 
calculada diretamente, obtendo

$$
\Xi(T, \mu, H)=\int_{-\infty}^{\infty} \int_{-\infty}^{\infty} \mathrm{e}^{+A f(x, y)} \mathrm{d} x \mathrm{~d} y
$$

onde

$$
f(x, y)=-\frac{x^{2}}{A}-\frac{y^{2}}{A}+\ln \left(1+\mathrm{e}^{\left(\alpha_{3} y+\alpha_{4} x+\alpha_{5}\right)}\left(\mathrm{e}^{\alpha_{1} x+\alpha_{2}}+\Omega \mathrm{e}^{-\alpha_{1} x-\alpha_{2}}\right)\right)
$$

A função $f(x, y)$ vai ser expandida em uma série de Taylor, ao redor do ponto de extremo $\left(x_{0}, y_{0}\right)$, obtido das relações

$$
\left.\frac{\partial}{\partial x} f\right|_{\left(x_{0}, y_{0}\right)}=0 \quad \text { e }\left.\quad \frac{\partial}{\partial y} f\right|_{\left(x_{0}, y_{0}\right)}=0
$$

O termo $e^{A f\left(x_{0}, y_{0}\right)}$ não é função de $x$ ou de $y$. Então podemos retirá - lo de dentro da integral, obtendo,

$$
\Xi(T, \mu, H)=\mathrm{e}^{A f\left(x_{0}, y_{0}\right)} \zeta \quad
$$

onde $\zeta$ é definido por

$$
\zeta=\iint_{-\infty}^{\infty} \mathrm{e}^{A\left(\left.\frac{1}{2} \frac{\partial^{2}}{\partial x^{2}} f\right|_{x_{0}, y_{0}}\left(x-x_{0}\right)^{2}+\left.\frac{1}{2} \frac{\partial^{2}}{\partial y^{2}} f\right|_{x_{0}, y_{0}}\left(y-y_{0}\right)^{2}+\left.\frac{\partial^{2}}{\partial x \partial y} f\right|_{x_{0}, y_{0}}\left(x-x_{0}\right)\left(y-y_{0}\right)\right)} \mathrm{d} x \mathrm{~d} y
$$

Com a equação 3.17, obtemos uma expressão para o potencial grande canônico, sendo que o termo $\ln \zeta$ é muito menor que $A f\left(x_{0}, y_{0}\right)$ e por isso ele 
vai ser desprezado,

$$
-\beta \Phi(T, \mu, H)=\ln \left(\mathrm{e}^{A f\left(x_{0}, y_{0}\right)} \zeta\right)=A f\left(x_{0}, y_{0}\right)+\ln \zeta \quad,
$$

onde

$$
x_{0}=\frac{A}{2} \frac{\left(\alpha_{4}+\alpha_{1}\right) \mathrm{e}^{\alpha_{1} x_{0}+\alpha_{2}}+\Omega\left(\alpha_{4}-\alpha_{1}\right) \mathrm{e}^{-\alpha_{1} x_{0}-\alpha_{2}}}{1+e^{\alpha_{3} y_{0}+\alpha_{4} x_{0}+\alpha_{5}}\left(\mathrm{e}^{\alpha_{1} x_{0}+\alpha_{2}}+\Omega \mathrm{e}^{-\alpha_{1} x_{0}-\alpha_{2}}\right)} e^{\alpha_{3} y_{0}+\alpha_{4} x_{0}+\alpha_{5}}
$$

e

$$
y_{0}=\frac{A}{2} \frac{\alpha_{3} e^{\alpha_{3} y_{0}+\alpha_{4} x_{0}+\alpha_{5}}\left(\mathrm{e}^{\alpha_{1} x_{0}+\alpha_{2}}+\Omega \mathrm{e}^{-\alpha_{1} x_{0}-\alpha_{2}}\right)}{1+e^{\alpha_{3} y_{0}+\alpha_{4} x_{0}+\alpha_{5}}\left(\mathrm{e}^{\alpha_{1} x_{0}+\alpha_{2}}+\Omega \mathrm{e}^{-\alpha_{1} x_{0}-\alpha_{2}}\right)} .
$$

Para analisar as relações é preciso de uma interpretação física dos parâmetros $\left(x_{0}, y_{0}\right)$ em termos da "magnetização" $M$,

$$
\begin{aligned}
M & =-\frac{\partial}{\partial H} \Phi \\
& =\frac{1}{\beta} A \frac{\partial f}{\partial H} \\
& =A \frac{\partial f}{\partial \alpha_{2}},
\end{aligned}
$$

e do quantidade de lipídios $N$,

$$
\begin{aligned}
N & =-\frac{\partial}{\partial \mu} \Phi \\
& =\frac{1}{\beta} A \frac{\partial f}{\partial \mu} \\
& =A \frac{\partial f}{\partial \alpha_{5}},
\end{aligned}
$$


dados por

$$
M=A \frac{e^{\alpha_{3} y_{0}+\alpha_{4} x_{0}+\alpha_{5}}\left(\mathrm{e}^{\alpha_{1} x_{0}+\alpha_{2}}-\Omega \mathrm{e}^{-\alpha_{1} x_{0}-\alpha_{2}}\right)}{1+e^{\alpha_{3} y_{0}+\alpha_{4} x_{0}+\alpha_{5}}\left(\mathrm{e}^{\alpha_{1} x_{0}+\alpha_{2}}+\Omega \mathrm{e}^{-\alpha_{1} x_{0}-\alpha_{2}}\right)}
$$

e

$$
N=A \frac{e^{\alpha_{3} y_{0}+\alpha_{4} x_{0}+\alpha_{5}}\left(\mathrm{e}^{\alpha_{1} x_{0}+\alpha_{2}}+\Omega \mathrm{e}^{-\alpha_{1} x_{0}-\alpha_{2}}\right)}{1+e^{\alpha_{3} y_{0}+\alpha_{4} x_{0}+\alpha_{5}}\left(\mathrm{e}^{\alpha_{1} x_{0}+\alpha_{2}}+\Omega \mathrm{e}^{-\alpha_{1} x_{0}-\alpha_{2}}\right)}
$$

Por inspeção, observa-se que

$$
x_{0}=\frac{1}{2}\left(\alpha_{4} N+\alpha_{1} M\right) \quad \text { e } \quad y_{0}=\frac{1}{2} \alpha_{3} N .
$$

Substituindo estas expressões em 3.15, temos

$$
\begin{gathered}
\beta \Phi(T, \mu, H)=\frac{1}{4}\left(\alpha_{3}^{2} N^{2}+\left(\alpha_{4} N+\alpha_{1} M\right)^{2}\right) \\
-A \ln \left(1+e^{\frac{1}{2}\left(\alpha_{3}^{2}+\alpha_{4}^{2}\right) N+\frac{\alpha_{1} \alpha_{4}}{2} M+\alpha_{5}}\left(\mathrm{e}^{\frac{1}{2}\left(\alpha_{1} \alpha_{4} N+\alpha_{1}^{2} M\right)+\alpha_{2}}+\Omega \mathrm{e}^{-\frac{1}{2}\left(\alpha_{1} \alpha_{4} N+\alpha_{1}^{2} M\right)-\alpha_{2}}\right)\right)
\end{gathered}
$$

Definimos densidade por $n=\frac{N}{A}$ e "magnetização" por área por $m=\frac{M}{A}$. Por analogia a sistemas magnéticos, denominamos a grandeza $m$ como a magnetização, mas no contexto de membrana, esta grandeza representa o estado médio das cadeias dos lipídios formadores da membrana. Se $m>0$, a maioria dos lipídios estão com as caudas ordenadas e se $m<0$, a maioria dos lipídios está com as caudas desordenadas. 
Finalmente temos

$$
\begin{aligned}
& \frac{\Phi(T, \mu, H ; m, n)}{A}=\frac{q}{2}\left(J m^{2}+K n^{2}+2 \Delta n m\right) \\
& -\frac{1}{\beta} \ln \left(1+\mathrm{e}^{\beta(q \Delta m+q K n+\mu)}\left(\mathrm{e}^{\beta(q J m+q \Delta n+H)}+\Omega \mathrm{e}^{-\beta(q J m+q \Delta n+H)}\right)\right)
\end{aligned}
$$

onde $m$ e $n$ devem satisfazer o seguinte sistema de equações de estado:

$$
\begin{aligned}
& m(m, n)=\frac{\mathrm{e}^{\beta(q \Delta m+q K n+\mu)}\left(\mathrm{e}^{\beta(q J m+q \Delta n+H)}-\Omega \mathrm{e}^{-\beta(q J m+q \Delta n+H)}\right)}{1+\mathrm{e}^{\beta(q \Delta m+q K n+\mu)}\left(\mathrm{e}^{\beta(q J m+q \Delta n+H)}+\Omega \mathrm{e}^{-\beta(q J m+q \Delta n+H)}\right)} ; \\
& n(m, n)=\frac{\mathrm{e}^{\beta(q \Delta m+q K n+\mu)}\left(\mathrm{e}^{\beta(q J m+q \Delta n+H)}+\Omega \mathrm{e}^{-\beta(q J m+q \Delta n+H)}\right)}{1+\mathrm{e}^{\beta(q \Delta m+q K n+\mu)}\left(\mathrm{e}^{\beta(q J m+q \Delta n+H)}+\Omega \mathrm{e}^{-\beta(q J m+q \Delta n+H)}\right)} .
\end{aligned}
$$

\section{Temperatura reduzida}

Definimos a temperatura reduzida $t$, que vai ser usada tanto na aproximação de campo médio como nas simulações numéricas, por

$$
t=\frac{1}{J} \frac{1}{\beta}
$$

\subsection{Fases ordenadas a temperatura nula}

À temperatura nula, esperamos que o sistema esteja no estado fundamental, isto é, em algum estado ordenado, de menor entropia possível. No caso de estados homogêneos, combinações possíveis são os estados de densidade nula $(\sigma=0)$ ou de densidade um $\left(\sigma^{2}=1\right)$, e, no segundo caso, poderíamos ter a 
"magnetização" igual a 1 ou a -1 . Para $J>0$, todos os sítios devem assumir os mesmos valores e para $J<0$ devem existir estados "anti - ferro", que não nos interessam, neste estudo.

Podemos encontrar as linhas que separam as fases ordenadas no espaço de parâmetros $K, \Delta, \mu$. Comparando as "energias" do sistema, $\phi=E-\mu N$ e Eq. 2.19, para as fases ordenadas consideradas, dadas por

$$
\begin{gathered}
\phi(T=0 ; n=0, m=0)=0 \\
\phi(T=0 ; n=1, m=1)=-J-2 \Delta-K-\mu
\end{gathered}
$$

e

$$
\phi(T=0 ; n=1, m=-1)=-J+2 \Delta-K-\mu .
$$

As linhas de separação são

$$
\begin{gathered}
-J+2 \Delta-K-\mu=0 \quad \text { (desordem - gás) } \\
-J-2 \Delta-K-\mu=0 \quad \text { (ordem - gás) } \\
\Delta=0 \quad \text { (ordem - desordem) }
\end{gathered}
$$

A consistência das linhas obtidas pode ser verificada a partir da solução de campo médio no limite de temperatura nula. Ou seja, as soluções devem satisfazer simultaneamente as equações de ponto fixo 3.29 e 3.30 no limite de 
$\beta \rightarrow \infty$

Por simplicidade, iremos definir

$$
\begin{aligned}
& D(m, n)=q \Delta m+q K n+\mu, \\
& B(m, n)=q J m+q \Delta n,
\end{aligned}
$$

e o par $(m, n)$ é reescrito na forma

$$
(m, n)=\frac{\mathrm{e}^{\beta(D(m, n)+B(m, n))}\left(1+(-1,+1) \Omega \mathrm{e}^{-2 \beta B(m, n)}\right)}{1+\mathrm{e}^{\beta(D(m, n)+B(m, n))}\left(1+\Omega \mathrm{e}^{-2 \beta B(m, n)}\right)}
$$

No limite de $\beta \rightarrow \infty$ as possíveis soluções dependem de $D(m, n)$ e $B(m, n)$. Dessa maneira

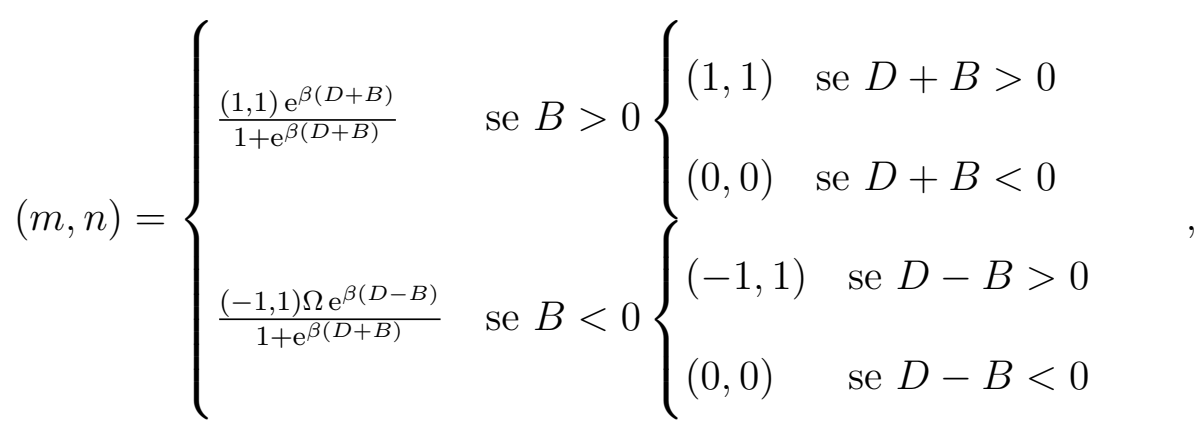

implicando que as condições de existência dos diversos estados são

- $\operatorname{ordenado},(1,1)$, implica que $\frac{2 q \Delta+q K+\mu+q J}{J}>0$.

- gás, $(0,0)$ implica que $\frac{\mu}{J}<0$

- desordenado, $(-1,1)$ implica que $\frac{-2 q \Delta+q K+\mu+q J}{J}>0$.

Na figura 3.1 é apresentado o diagrama de fases no espaço de parâmetros da energia, em temperatura nula, para $J>0$. Para observar a transição 


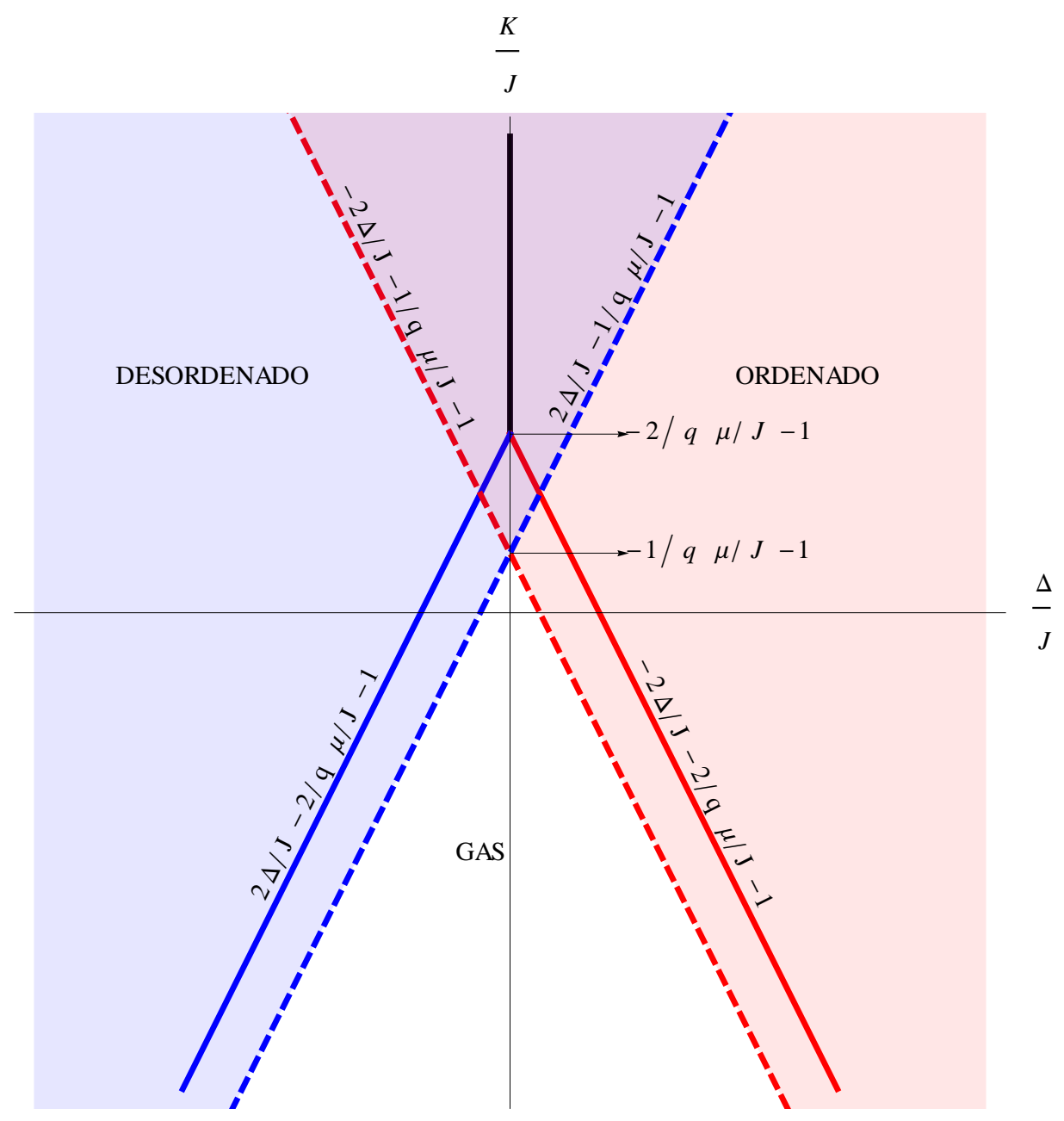

Figura 3.1: Diagrama de fase a temperatura nula, $J>0$ e $\mu<0$. As linhas tracejadas representam o limite de existência das soluções de campo médio e as linhas cheias a transição entre os estados ordenado, desordenado e gasoso.

ordem - desordem, os parâmetros $\frac{\Delta}{J}, \frac{K}{J}$ e $\frac{\mu}{J}$ devem ser ajustados para que em baixas temperaturas o sistema esteja no estado ordenado. Isto implica em tomar $\Delta>0$ e

$$
K>1-2 \Delta-2 \frac{\mu}{q}
$$

Nestas condições, o potencial químico de transição gás - líquido ordenado 
a temperatura nula, $\mu^{*}$, é determinado exatamente,

$$
\mu^{*}=-\frac{q}{2}(J+K+2 \Delta)
$$

permitindo localizar a região de interesse no diagrama de fases.

\section{Escolha dos parâmetros do modelo}

Gostaríamos que os parâmetros $\frac{\Delta}{J}$ e $\frac{K}{J}$ fossem ajustados com dados experimentais. Neste trabalho, os valores desses parâmetros são escolhidos arbitrariamente, sob algumas condições de origem qualitativa, a fim de se observar a transição ordem - desordem. Todos os parâmetros devem ter a mesma ordem de grandeza, para poderem ser relevantes à dinâmica do sistema. Os parâmetros reduzidos são

$$
\frac{\Delta}{J}=\frac{\epsilon_{G}-\epsilon_{F}}{\epsilon_{G}+\epsilon_{F}-2 \epsilon_{G F}}
$$

$\mathrm{e}$

$$
\frac{K}{J}=\frac{\epsilon_{G}+\epsilon_{F}+2 \epsilon_{G F}}{\epsilon_{G}+\epsilon_{F}-2 \epsilon_{G F}}
$$

Lembrando que $\epsilon_{G}, \epsilon_{F}$ e $\epsilon_{G F}$ são as energias de interação efetiva entre pares, respectivamente: de pares com cadeias de ambos os lipídios no estado ordenado; de pares com cadeias de ambos os lipídios no estado desordenado; e de pares em que um dos lipídios tem suas cadeias no estado ordenado e o outro tem suas cadeias no estado desordenado. A interação entre as caudas lipídicas é efetivamente atrativa, implicando que os parâmetros $\epsilon_{G}, \epsilon_{F}$ e $\epsilon_{G F}$ são estritamente positivos e, como a distância entre lipídios ordenados é 
a priori menor do que a distância entre lipídios desordenados, temos que $\epsilon_{F}<\epsilon_{G}$. Implicando que, em termos dos nossos novos parâmetros, $\frac{\Delta}{J}>0$ e $\frac{K}{J}>1$.

Os primeiros resultados foram obtidos definindo os parâmetros

$$
\frac{\Delta}{J}=0.9 \quad \text { e } \quad \frac{K}{J}=1.1
$$

que satisfazem as condições citadas.

\subsection{Métodos numéricos}

As equações 3.29 e 3.30 formam um sistema de equações acopladas de difícil resolução. Este problema pode ser descrito como um problema de zero de uma função bidimensional, ou como uma equação de ponto fixo bidimensional.

Inicialmente, foi tentado usar o método iterativo, que não foi capaz de encontrar todas as soluções. Em seguida, foi tentado usar o método de Newton - Raphson, que também não teve sucesso em obter todas as soluções. Na figura 3.2 são apresentados gráficos de contorno do sistema de duas equações acopladas, onde as soluções são dadas nos pontos em que as linhas se cruzam. No gráfico a $t=0,001$, fica claro que no limite de temperatura nula há dificuldades de se determinar numericamente algumas das soluções. Nesta região a derivada usada no método de Newton - Raphson tende a zero e o ruido da imprecisão numérica se torna relevante, por isso o método falha

Para contornar este problema, foi decidido encontrar as soluções na força 

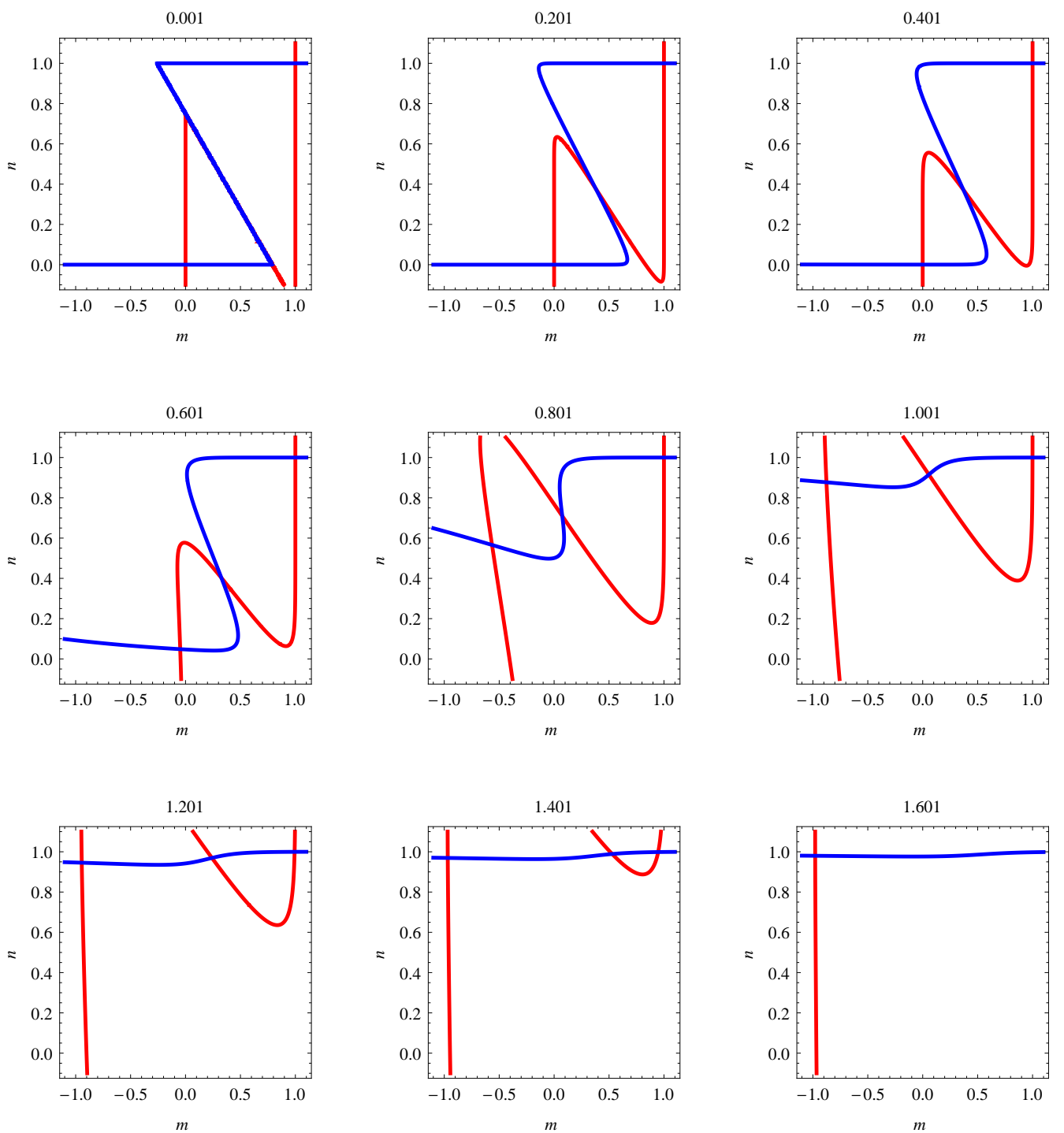

Figura 3.2: Gráficos de contorno do conjunto de equações de ponto fixo $m(m, n)$ e $n(m, n)$ para $\frac{\mu}{J}=-6$ e temperaturas de 0,001 até 1,601. A linha vermelha é formada pelo conjuntos de pontos $(m, n)$ tais que satisfazem a equação $m=m(m, n)$, e a linha azul pelo conjunto de pontos que satisfazem a equação $n=n(m, n)$. As soluções do sistema de equações são os ponto que satisfazem simultaneamente a eq. 3.29 e 3.30, ou seja, os pontos onde as linhas vermelhas e azuis se cruzam. No gráfico à temperatura $t=0.001$, as duas linhas são quase duas retas paralelas, no limite de temperatura nula há dificuldades de se determinar numericamente algumas das soluções e é preferível obter resultados analíticos. 
bruta. O método consiste simplesmente de varrer todo o intervalo de valores possíveis de $m$ e $n$ e selecionar as regiões nas quais observa-se uma mudança no sinal de $(m-n)$. Depois é usado o método iterativo como critério para avaliar se a região está próxima de um ponto fixo. O erro desse método, 0,001, é o tamanho do incremento usado para varrer os valores de $m$ e $n$.

Na figura 3.3 são traçados gráficos da magnetização, densidade e energia livre para todas as soluções encontradas pelo método numérico. O estado de equilíbrio é o estado de menor energia livre. No gráfico de energia livre observa-se que o sistema passa de um estado gel para um estado fluido por volta de $t_{m}=0,96$, sem passar por estado intermediários, caracterizando uma transição de primeira ordem.

\subsection{Diagrama de fases}

A escolha do parâmetro de ordem é arbitrária. Porém, no desenvolvimento da abordagem de campo médio no ensemble grande canônico, surgiram dois parâmetros de ordem a priori independentes: a quantidade de lipídios e o parâmetro relacionado com a ordem dos lipídios, que denominaremos de "magnetização". Definimos então a quantidade de lipídios por sítio de densi-

dade $n$ e, analogamente, a "magnetização" por sítio $m$. Poderíamos também usar como parâmetro de ordem a "magnetização" por partícula,

$$
\frac{m}{n}=\frac{1}{N} \sum_{i} \sigma_{i}
$$

mas neste trabalho preferimos a "magnetização" por sítio. 

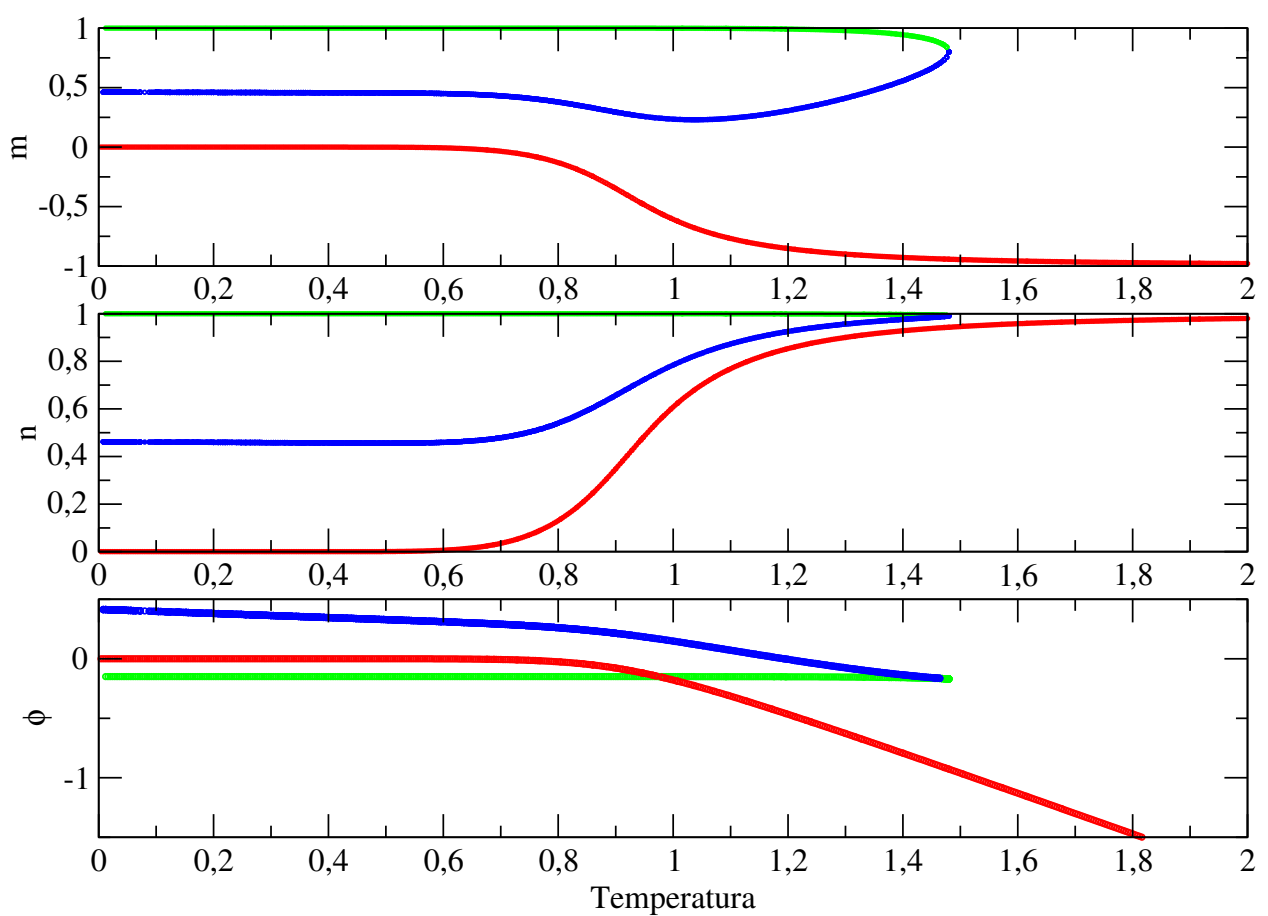

Figura 3.3: Magnetização $m$, densidade $n$ e energia livre $\phi$ de todas as soluções de campo médio para $\mu / J=-7,2, \Delta / J=0,9 \mathrm{e} K / J=1,1$. Observa-se que a temperatura baixa, as equações 3.29 e 3.30 são satisfeitas por 3 soluções distintas, discriminadas pelas cores verde, azul e vermelho. A solução de equilíbrio é aquela de menor energia livre, ou seja, a solução $m=1$ e $n=1$ (linha verde) abaixo da temperatura de transição e a solução (linha vermelha) $m<0$ e $n<1$ acima da temperatura de transição.

Na figura 3.4 é apresentado um diagrama de fase $t-\mu$ preliminar e as figuras seguintes representam cortes que ilustram a construção do diagrama. No diagrama $t-\mu$ aparecem as 3 fases esperadas: ordenada, desordenada e gasosa.

A baixa temperatura e potencial químico alto, identificamos esta região com a fase líquido ordenado, caracterizada por lipídios com cadeias ordenadas ocupando a menor área possível. Aumentando a temperatura, observa-se a transição líquido ordenado - líquido desordenado, com um aumento abrupto da área ocupada por lipídio e as caudas assumem configurações desordenadas. 


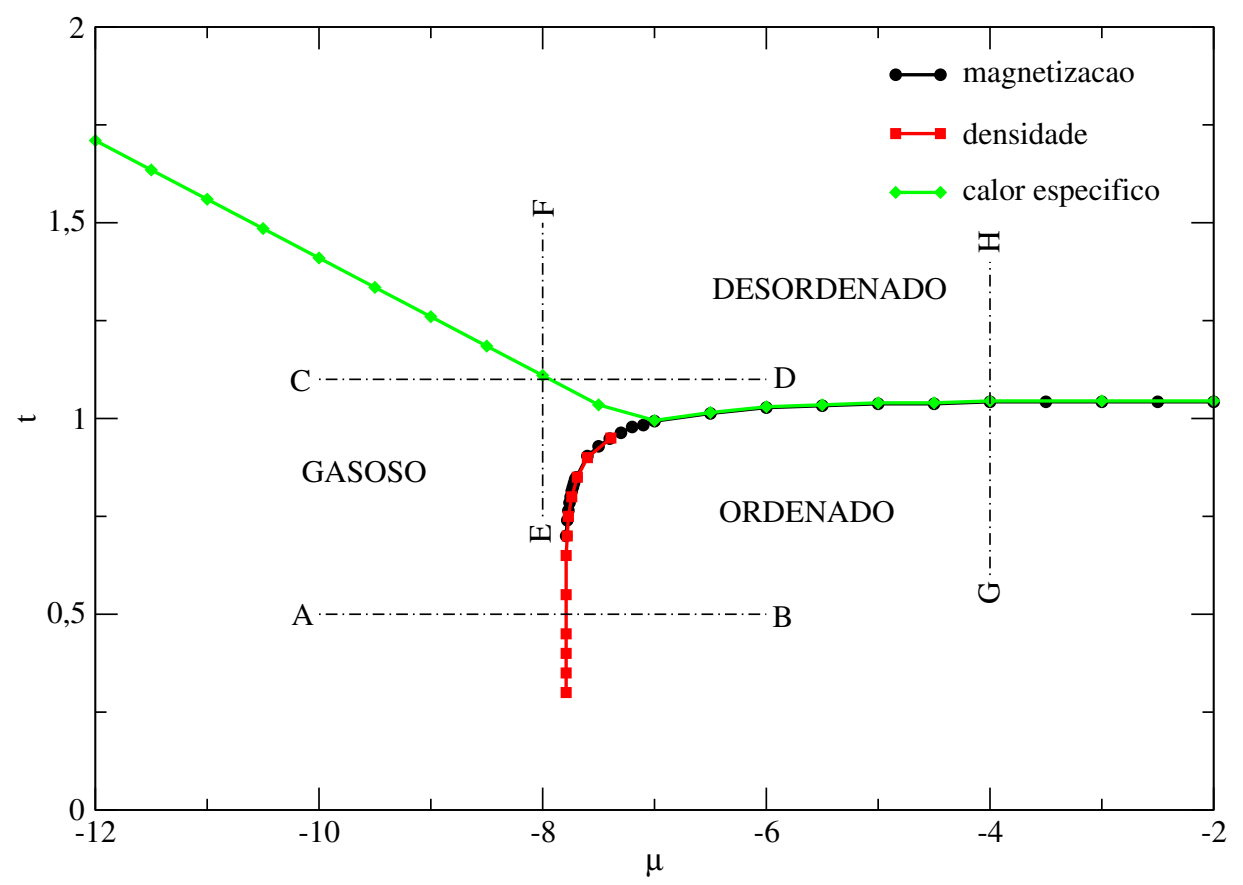

Figura 3.4: Diagrama de fase preliminar para $\Delta / J=0,9$ e $K / J=1,1$. As curvas foram obtidas por aproximação de campo médio a critérios diferentes: a curva identificada por magnetização mostra as temperaturas onde ocorrem descontinuidade na magnetização por sítio, e a curva identificada por densidade mostra os potenciais químicos onde correm descontinuidade na densidade. A curva identificada por calor específico foi traçada nas temperaturas onde ocorrem o máximo global do calor específico a potencial químico constante. É um resultado exploratório, os fins das curvas não representam pontos críticos. A "magnetização", densidade e energia livre nos segmentos $A B, C D, E F$ e GH, são apresentados nas figuras $3.5 \mathrm{a}, 3.5 \mathrm{~b}, 3.5 \mathrm{c}$ e $3.5 \mathrm{~d}$, respectivamente.

A partir do estado líquido ordenado, abaixando o potencial químico, observase a transição líquido ordenado - gás, com uma queda abrupta da densidade, ou um aumento abrupto da área por lipídio.

Está representada no diagrama de fase também uma linha de máximos do calor específico a potencial químico constante. Esta curva avança para uma região onde nenhum dos dois parâmetros de ordem apresentam descontinuidade.

Na figura 3.5a, é apresentado isotermas de "magnetização", densidade 

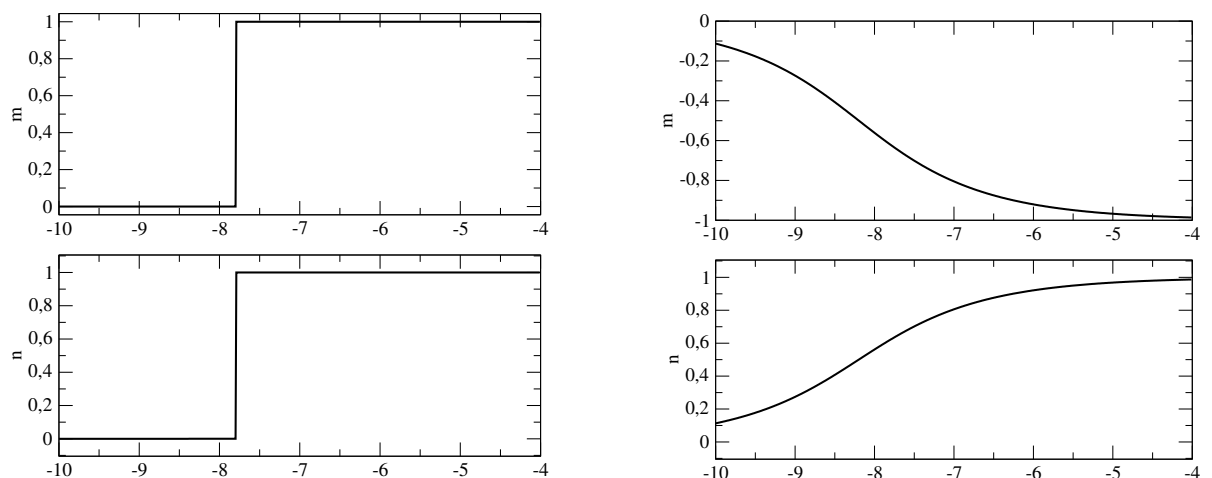

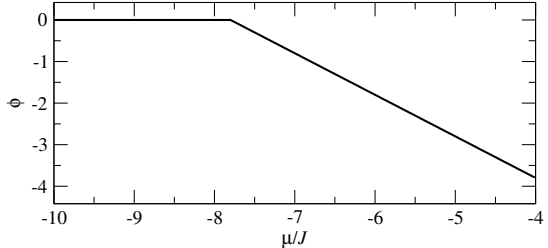

(a) corte $\mathrm{AB}$
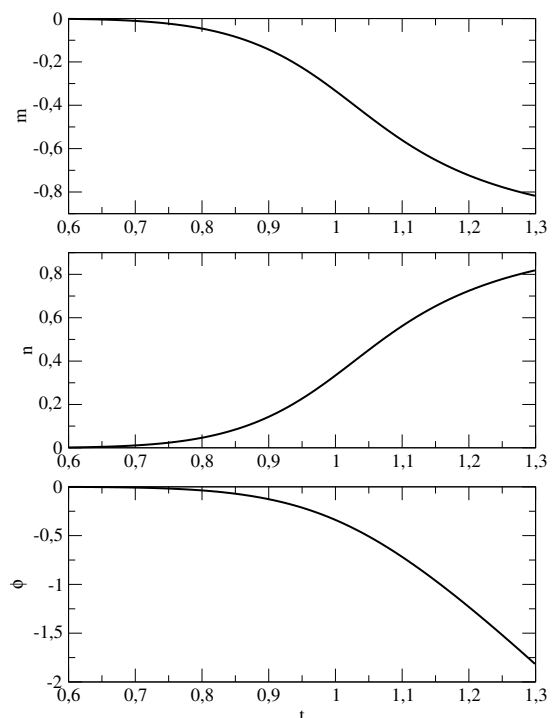

(c) corte $\mathrm{EF}$

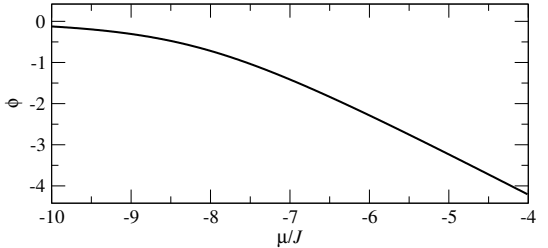

(b) corte CD
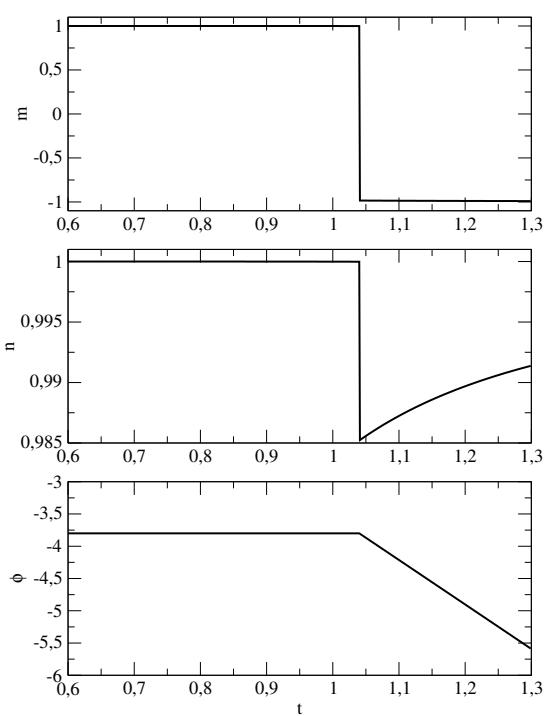

(d) corte GH

Figura 3.5: Curvas de "magnetização" por sítio, densidade e energia livre por sítio dos cortes indicados no diagrama de fase 3.4. 


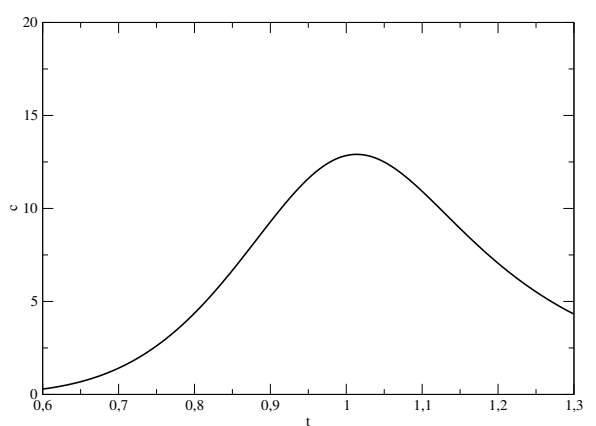

(a) corte EF

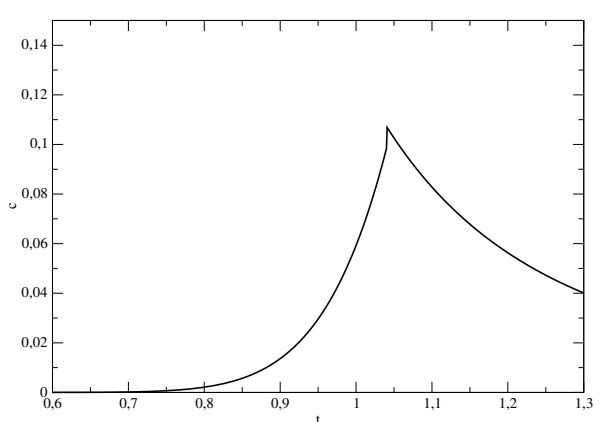

(b) corte GH

Figura 3.6: Calor específico como função da temperatura a potencial químico constante dos cortes indicados no diagrama de fase 3.4 .

e energia livre em função do potencial químico, corte AB. Observa-se a transição gás - líquido ordenado, caracterizada pela descontinuidade na densidade. Analogamente, no corte CD (figura 3.5b), as curvas para temperatura acima da temperatura de transição líquido ordenado - líquido desordenado não apresentaram nenhuma descontinuidade.

A figura 3.5c, referente ao corte EF, confirma a inexistência de descontinuidade dos parâmetros de ordem, já observada na figura 3.5b, referente ao corte CD. Para potencial químico maior que o potencial químico de transição gás - líquido ordenado, no segmento GH (figura 3.5d), observa-se a transição líquido ordenado - líquido desordenado, com descontinuidades na densidade e na "magnetização" por sítio.

Os cortes EF e GH são examinados também sob o ponto de vista do calor específico a potencial químico constante, apresentados respectivamente nas figuras 3.6a e 3.6b. O corte GH apresenta um pico no calor específico na temperatura das descontinuidades na densidade e na "magnetização". Já o corte EF apresenta um máximo, mas não um pico, como no corte GH. 


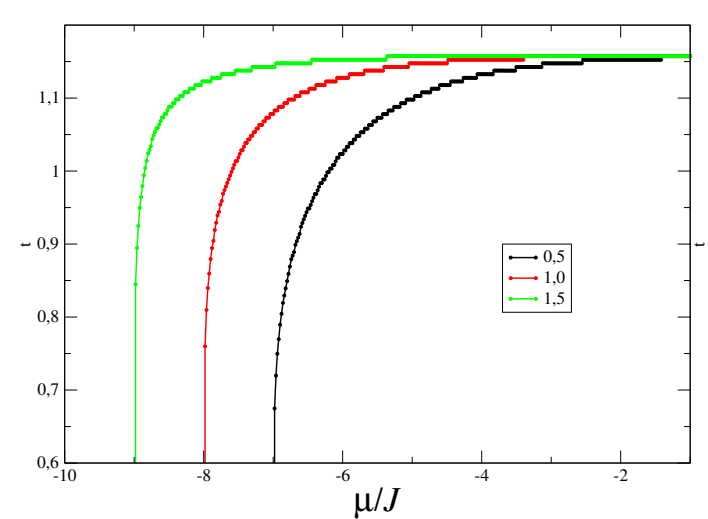

(a) Variando $K$

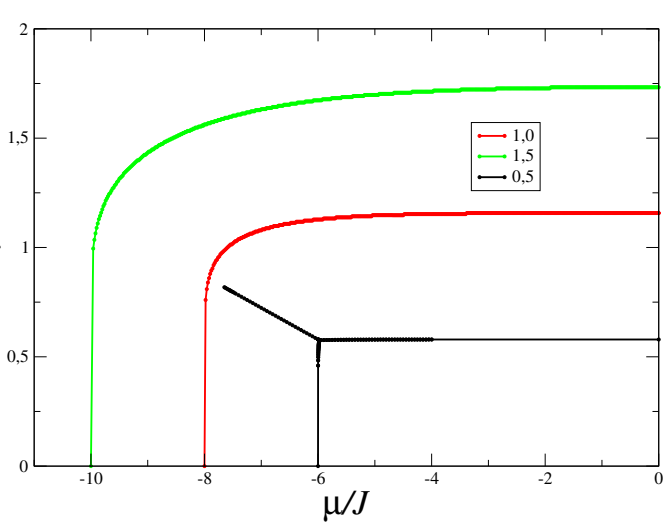

(b) Variando $\Delta$

Figura 3.7: Diagrama de fases: (a) para $\Delta / J=1$ e $K / J=0,5,1$ e 1,5, e (b) para $K / J=1$ e vários valores de $\Delta / J=0,5,1$ e 1,5 .

No caso do diagrama de fases da fig. 3.4, a passagem do estado gasoso para o estado de líquido - desordenado é continua. No entanto, é razoável esperar uma linha de coexistência separando uma fase líquida de uma fase gasosa. Por isso, decidimos investigar a existência de uma linha de transição para outro valores de parâmetros do modelo.

\section{Variando os parâmetros}

Na figura 3.7 apresentamos diversos diagramas de fases para diferentes valores dos parâmetros $\Delta$ e $K$. As figuras seguintes, 3.8 e 3.9. ilustram o estudo de cortes desdes diagramas.

Na figura 3.7a, observamos que a variação do parâmetro $K$ desloca a linha gás - líquido ordenado. A medida que $K$ cresce, a transição se desloca para valores menores do potencial químico.

Na figura 3.7b, observamos o deslocamento tanto da linha líquido - gás, como da linha ordem - desordem. Aumentando $\Delta$ para $K$ constante, a linha 
gás - líquido desloca-se para potencial químico menor e a linha ordem desordem desloca-se para temperaturas maiores. Além disso, para o menor valor de $\Delta$ apresentado, $\Delta=0.5$, surge uma linha de coexistência entre as fases gás e líquido desordenado. Dessa forma, o diagrama apresenta também um ponto triplo de coexistência das três fases.

O comportamento do modelo no caso em que as 3 fases são bem definidas, $\Delta=0,5$ na fig. 3.7b, é explorado com mais detalhes nas figuras 3.8 e 3.9. A figura 3.8a ilustra as descontinuidades da magnetização e da densidade na linha ordem - desordem. A figura 3.8b apresenta estas descontinuidades na linha de coexistência líquido - gás.

A fig. 3.9a apresenta um comportamento mais complexo do modelo nas proximidades do ponto triplo. $\mathrm{O}$ aumento da temperatura a potencial químico fixo produz uma descontinuidade da magnetização positiva para uma magnetização nula, seguida de uma transição para magnetização negativa. Concomitantes com estas transições na magnetização, ocorre uma transição de densidade alta para uma densidade próxima de zero, seguida de uma transição para uma densidade menor do que um. Isto significa que, sob a variação da temperatura, ocorre uma transição do estado ordenado para o estado gasoso, seguida de uma transição do estado gasoso para o estado desordenado.

Na figura 3.9b observa-se uma transição de magnetização nula para magnetização negativa, concomitante com uma transição de densidade próxima de zero para uma densidade próximo de um. Portanto, este diagramas descrevem a transição gás - líquido desordenado.

Devido a existência de coexistência entre as fases gás e líquido desorde- 

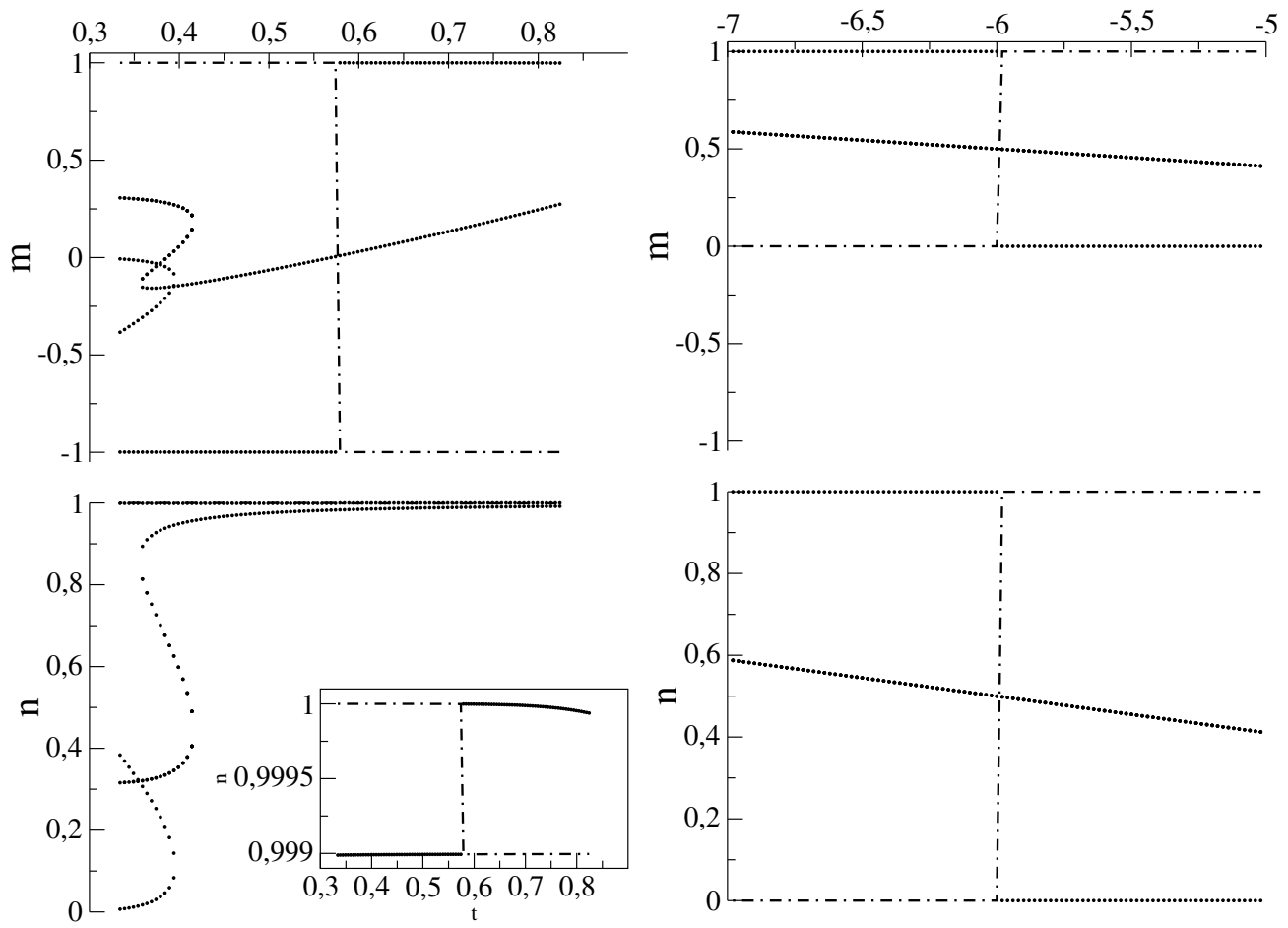

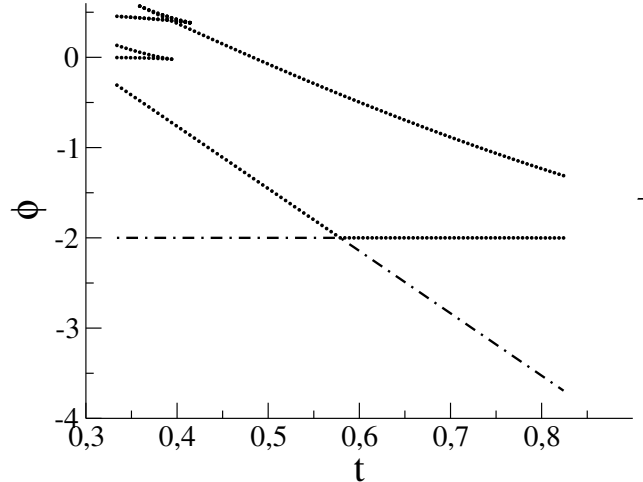

(a)

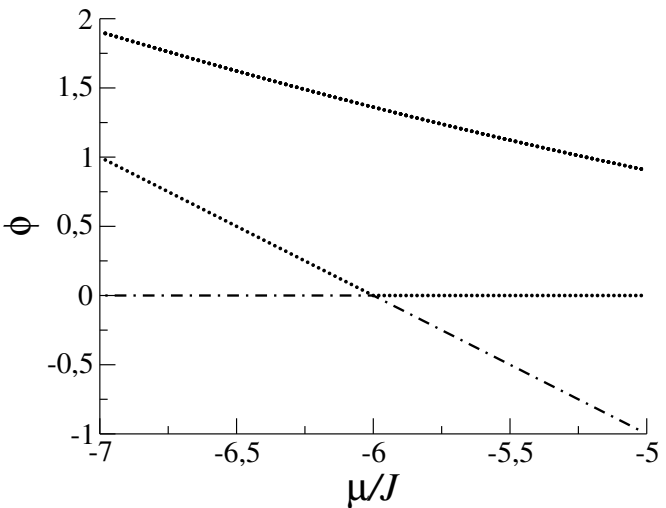

(b)

Figura 3.8: Gráficos de "magnetização" por sítio $m$, densidade $n$ e energia livre $\phi$. Os parâmetros comuns são $\Delta / J=0,5, K / J=1$ e $\Omega=1000$. A linha traço-ponto indica a solução de equilíbrio: (a) transição ordem - desordem a potencial químico fixo a $\mu / J=-4$ e variando a temperatura; (b) transição líquido - gás a temperatura fixa em $t=0,2 \mathrm{e}$ variando o potencial químico. 

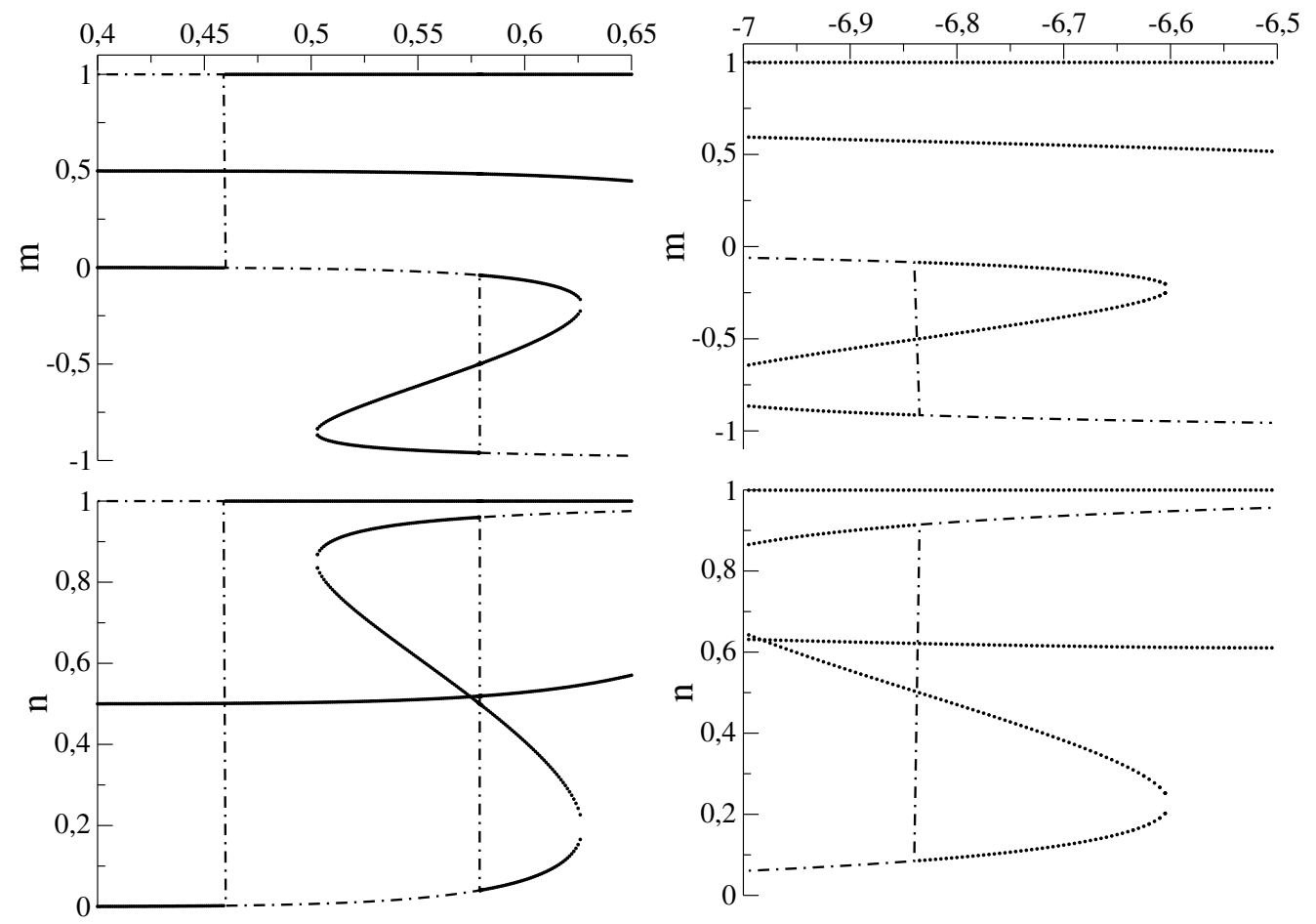

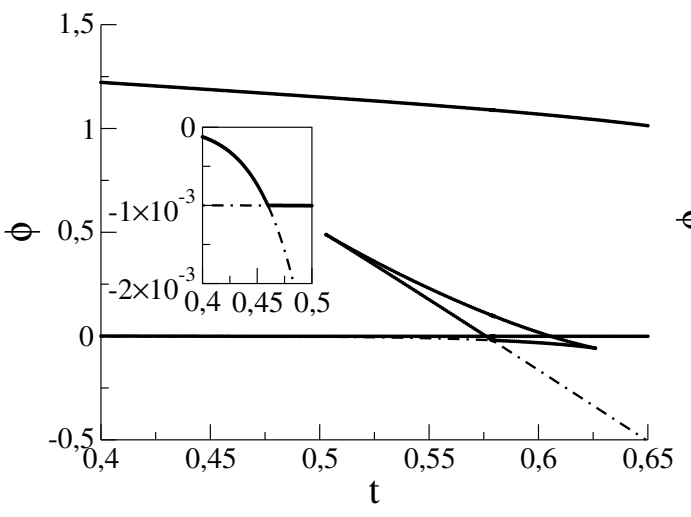

(a)

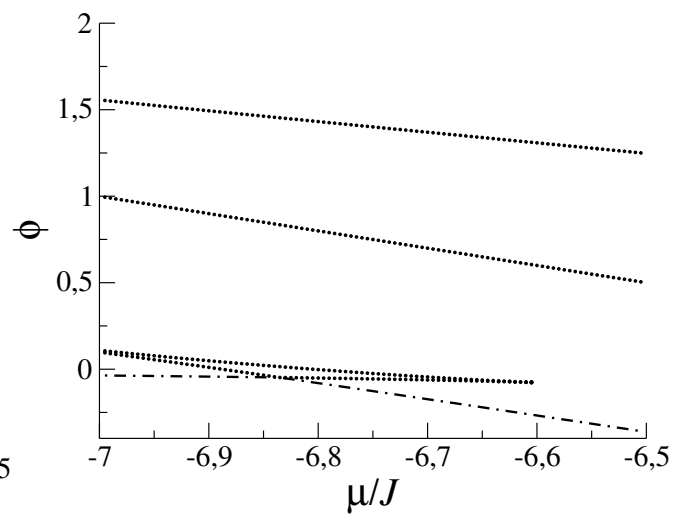

(b)

Figura 3.9: Gráficos de "magnetização"por área $m$, densidade $n$ e energia livre $\phi$. Os parâmetros comuns são $\Delta / J=0,5, K / J=1$ e $\Omega=1000$. A linha traço-ponto indica a solução de equilíbrio: (a) transição "líquido ordenado" - gás - "liquido desordenado" variando a temperatura e fixando o potencial químico em $\mu=-5,999$, um valor marginalmente maior que o potencial químico de transição líquido - gás a temperatura nula $\mu_{0}=-6$; (b) transição gás - "líquido desordenado" a temperatura fixa em $t=0,7$, acima da temperatura de transição ordem - desordem, e variando o potencial químico. 
nado, e portanto, de um ponto triplo e de um possível comportamento reentrante, exploramos para diferente valores de $\Delta$, proximos a $\Delta=0,5$. Este estudo é apresentado na figura 3.10, onde observa-se que a temperatura do ponto crítico, não tem um comportamento monotônico com o crescimento de $\Delta$. A temperatura crítica aumenta, depois diminui e finalmente desaparece, pois a linha de coexistência gás - líquido desordenado desaparece.

Pode-se inferir que, na competição das duas linhas gás - líquido e ordem desordem, o ponto crítico aproxima-se do triplo e depois ambos desaparecem.

O exame da expressão da energia do modelo, eq. 2.19, permite explicar os deslocamentos das linha gás - líquido e ordem - desordem. O parâmetro $K$, coeficiente do termo biquadrático, $\sigma_{i}^{2} \sigma_{j}^{2}$, favorece as fases densas, com $\sigma= \pm 1$ e, por isso, desloca a transição gás - líquido ordenado.

O parâmetro $\Delta$, coeficiente da interação cúbica, favorece a fase líquido ordenado com $\sigma=-1$ e, por isso, desloca as duas linhas, gás líquido e ordem - desordem, ampliando ou reduzindo a fase de líquido ordenado.

Na fig. 3.10, apresentamos alguns diagramas de fases para alguns valores de $\Delta$. Para $\Delta=\{0,5 ; 0,55 ; 0,6\}$, é observado a existência da transição gás líquido desordenado, e portanto, é observado também um ponto triplo.

\section{Diagrama de fases pressão - temperatura}

O potencial químico não é uma grandeza mensurável em laboratório. Por isso, é interessante desenharmos diagramas de fase pressão - temperatura.

A pressão pode ser obtida a partir do potencial químico por meio da 


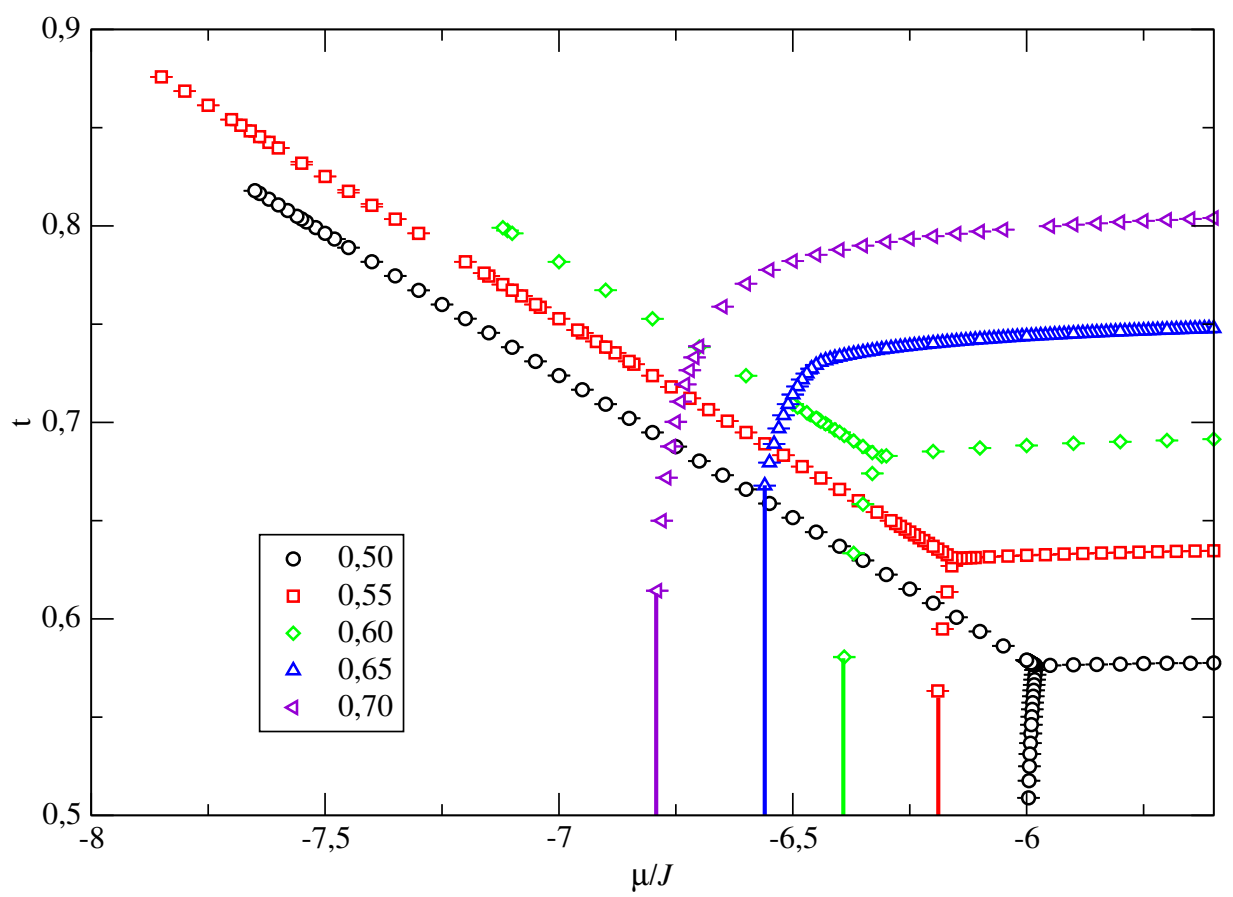

Figura 3.10: Diagrama de fases para $K / J=1$ e alguns valores de $\Delta / J$. Três linhas de coexistência separam as fases ordenada e desordenada, as fases ordenada e gasosa e as fases desordenada e gasosa. As três fases coexistem em um ponto triplo. A linha de coexistência gás-líquido desordenado termina em um ponto crítico.

integração numérica da relação de Gibbs - Duhem,

$$
-S d T+A d \Pi-N d \mu=0,
$$

$\mathrm{Ou}$

$$
d \mu=-s d T+\frac{1}{n} d \Pi
$$

onde $s$ é a entropia por partícula, П a pressão lateral e as outras variáveis foram definidas anteriormente. Para temperatura constante, podemos escrever

$$
\Pi(t, \mu)=\int_{-\infty}^{\mu} n\left(t, \mu^{\prime}\right) d \mu^{\prime} .
$$




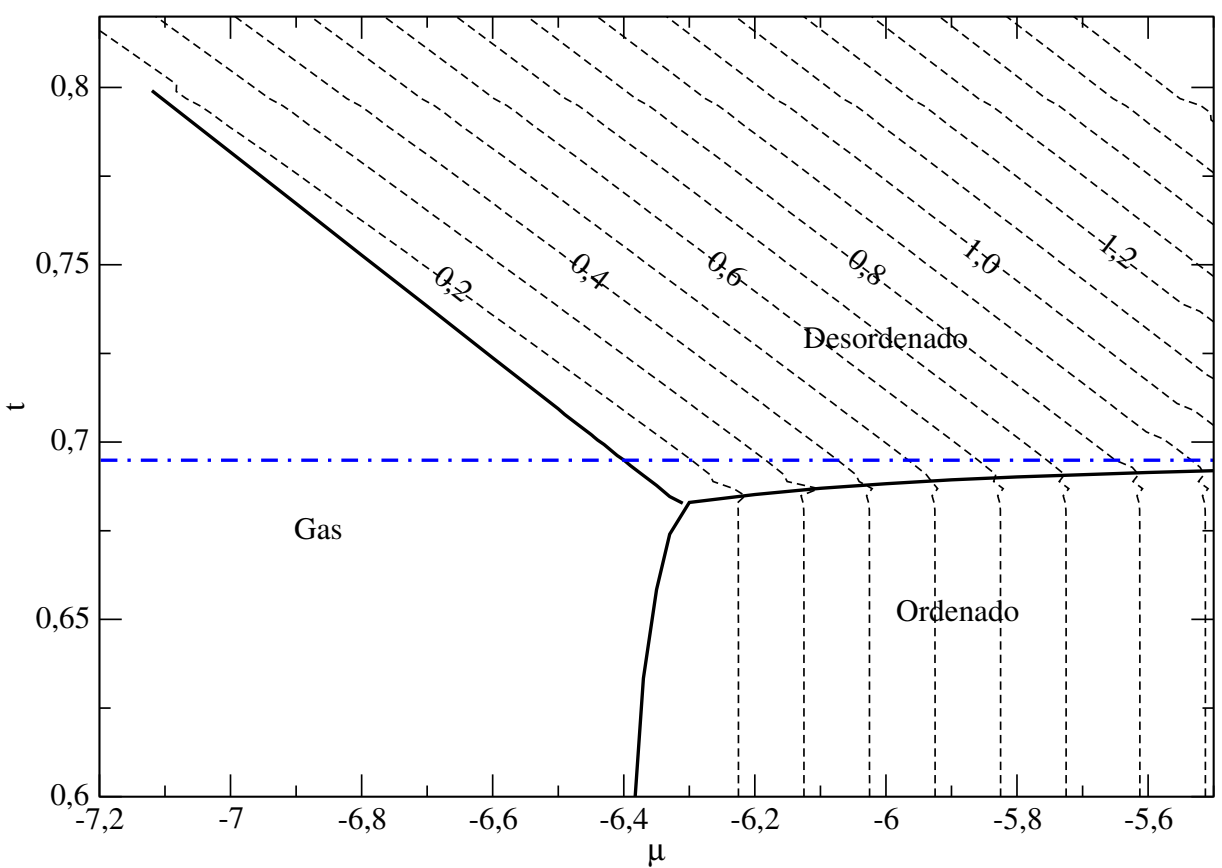

(a)

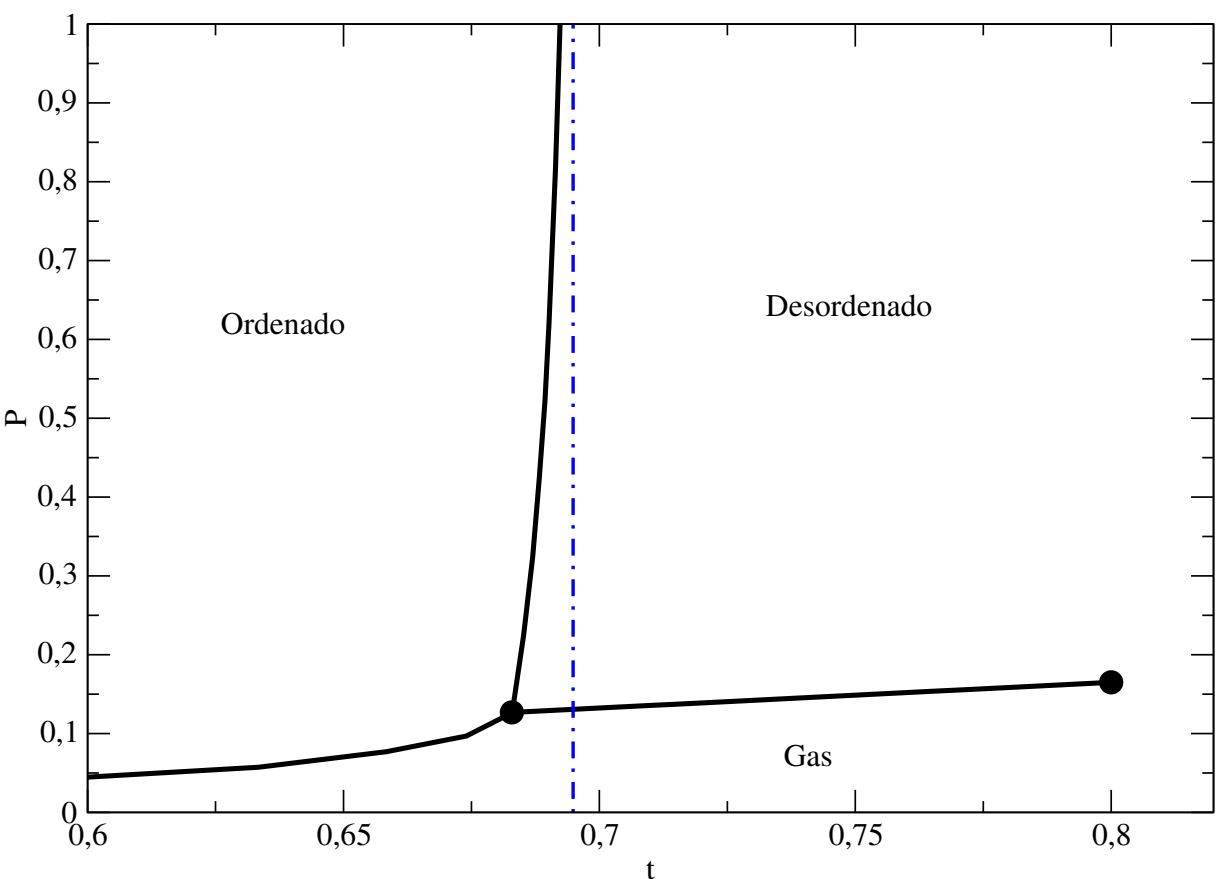

(b)

Figura 3.11: Diagramas de fase temperatura - potencial químico (a) e pressão - temperatura (b). Os parâmetros são $\Delta / J=0,6, K / J=1$ e $\Omega=1000$. Na figura (a), as linhas tracejadas são isobáricas. Em cada figura, a linha traço - ponto em azul indica a temperatura de transição ordem - desordem em alta densidade, potencial químico alto, $t^{*}$. 


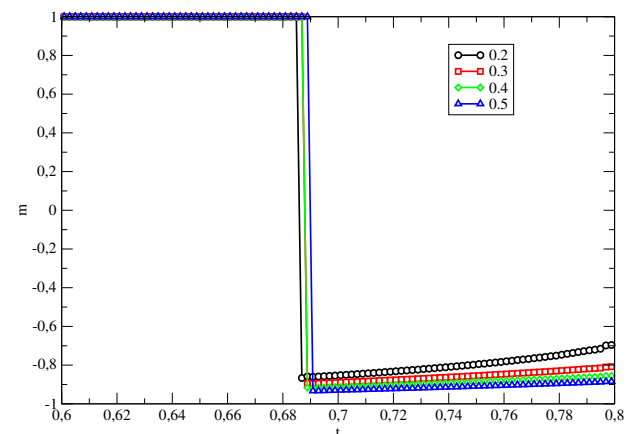

(a)

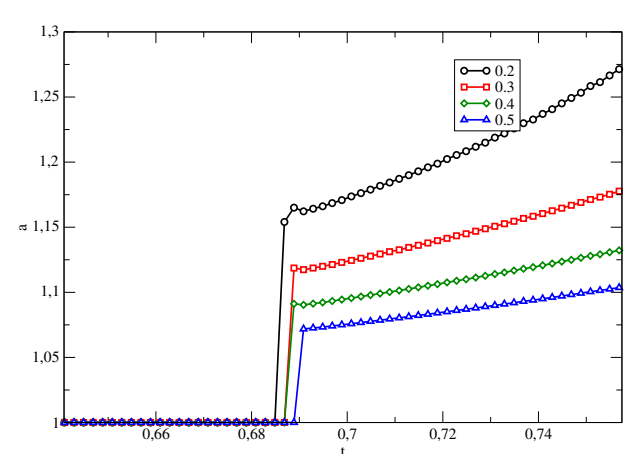

(b)

Figura 3.12: Isobáricas de "magnetização" (a) e área (b) em função da temperatura para várias pressões laterais. Os parâmetros são $\Delta=0,6, K=1$ e $\Omega=1000$.

Na prática, integramos a densidade a partir de valores suficientemente baixos de $\mu$, nos quais a densidade é praticamente nula, até o valor de $\mu$ no qual a pressão lateral $\Pi(\mu)$ será associada. O mapeamento potencial químico - pressão lateral ao longo do diagrama de fases é apresentado em termos de isobáricas na figura 3.11a para $\Delta=0,6$ e $K=1$. O diagrama correspondente pressão - temperatura está na figura 3.11b. Na figura 3.11b apresentamos o diagrama pressão lateral - temperatura.

Algumas curvas de magnetização, grandeza que descreve o estado médio das caudas hidrofóbicas, e de área por lipídio, como função da temperatura, ao longo da transição ordem - desordem, a pressão constante, estão apresentadas na figura 3.12a e 3.12b. Estas figuras podem ser comparadas às figura experimentais $1.2 \mathrm{a}$ e 1.3 .

À pressão lateral fixa, as curvas de área em função da temperatura apresentam uma descontinuidade, figura 3.12b. Concomitantemente, a "magnetização", que descreve o estado médio das caudas hidrofóbicas, apresenta também um salto nas mesmas temperaturas. 


\section{Monocamadas de Langmuir}

A inspeção do diagrama de fases pressão - temperatura, figura 3.11b, mostra que o modelo DLG pode ser útil para representar as transições de fases de monocamadas lipídicas de Langmuir (ver Capítulo 1). Nestes sistemas, a compressão isotérmica produz uma transição da fase gasosa para a fase de líquido expandido, com cadeias desordenadas, seguida de uma transição para o estado líquido condensado, de cadeias estendidas. Esta sequência corresponde a uma sequência de densidade muito baixa — densidade intermediária — densidade alta. Esta sequência ocorre no diagrama de fases da figura 3.11b para uma transformação isotérmica nas proximidades e à direita do ponto triplo, e à esquerda da temperatura de transição líquido ordenado - líquido desordenado denso $t^{*}$, linha tracejada do diagrama $\Pi / J-t$.

Na figura 3.13, são apresentadas alguma isotermas de pressão lateral em função da área por lipídio. Para temperaturas mais baixas, estão presentes as duas transições, e para temperatura mais alta, a transição entre as duas fases líquidas desaparece. Esta figura pode ser comparada à figura experimental 3.14. Do ponto de vista experimental, identifica-se o desaparecimento da transição com um ponto crítico. Em nosso modelo, a extinção desta transição ocorre em temperaturas acima da temperatura limite para densidade de empacotamento máximo. A discrepância merece mais investigação.

Entendemos que monocamadas de Langmuir são, de maneira simplificada, bicamadas lipídicas sob vínculos termodinâmicos diferentes. Descrevendo o diagrama de fase $\Pi-t$, figura $3.11 b$, pelo jargão de monocamadas de Lang- 


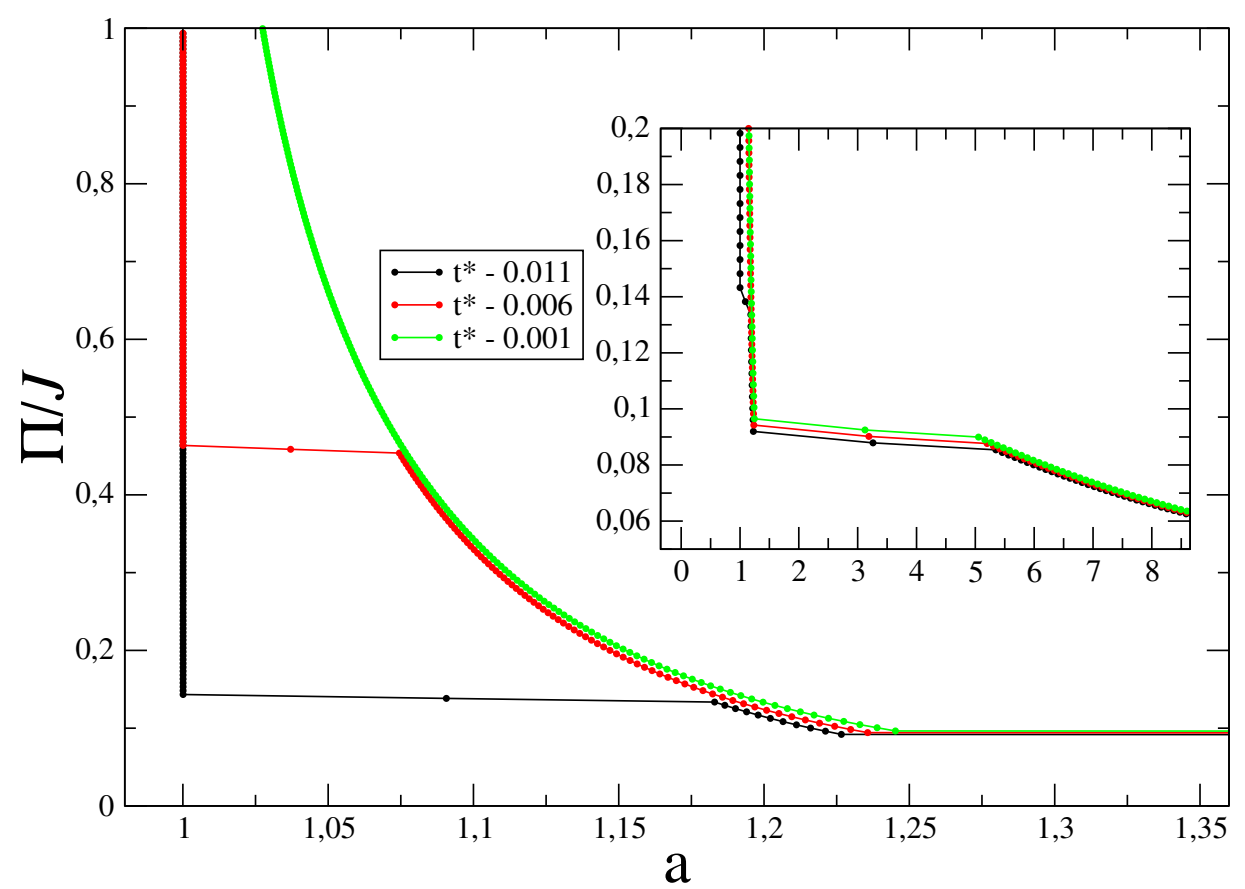

Figura 3.13: Isotermas pressão $\Pi / J$ em função da área transversal por lipídio a em diferentes temperaturas. A transição líquido ordenado - líquido desordenado é observada para as duas temperaturas mais baixas e a transição líquido desordenado - gás pode ser observada no detalhe da figura para áreas grandes.

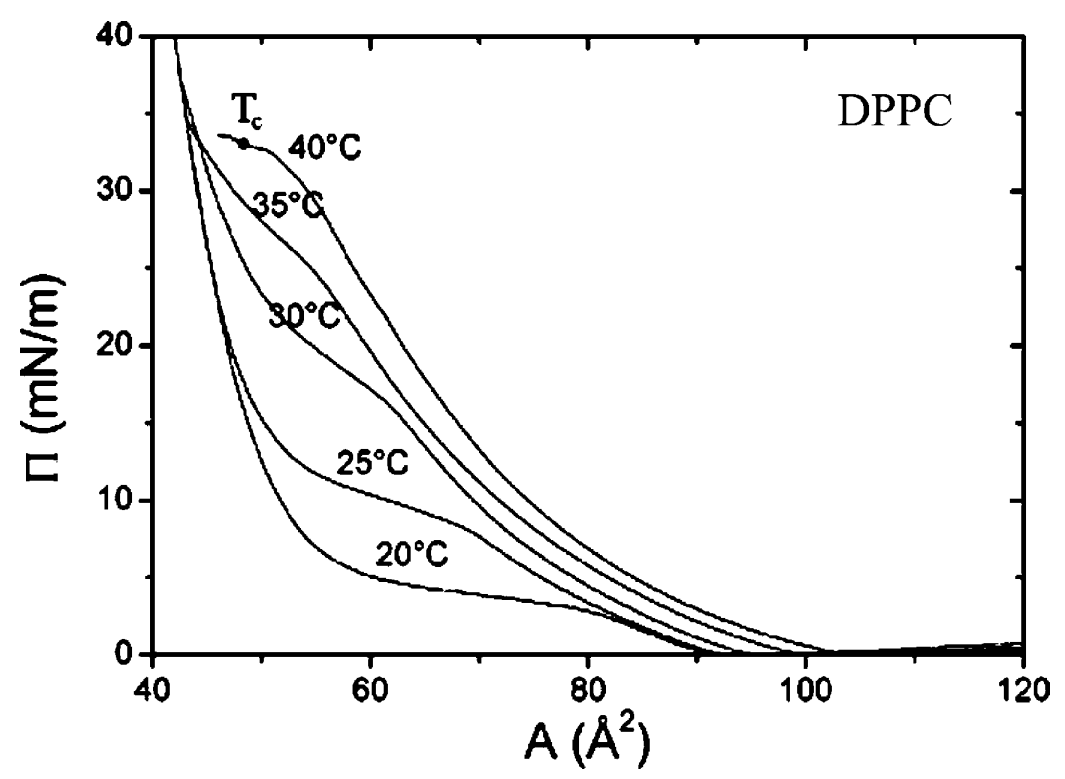

Figura 3.14: Isotermas de pressão lateral $\Pi$ versus área média por lipídio. para monocamada formada de DPPC. Figura copiada da referencia [18]. 
muir, diríamos que: abaixo da temperatura do ponto triplo, as isotermas apresentam regiões de coexistência de fases gás - líquido condensado; entre a temperatura do ponto triplo e a temperatura de transição ordem - desordem de alta densidade $t^{*}$, as isotermas apresentam coexistências das fases gás - líquido expandido e líquido expandido - líquido condensado; acima da temperatura de transição ordem - desordem a alta densidade e abaixo da temperatura crítica, apresenta a coexistência das fases gás - líquido expandido; acima da temperatura crítica, a fase gás e a fase líquido expandido são indistinguíveis. 


\section{Capítulo 4}

\section{Modelo DLG: simulações}

\section{numéricas}

No capítulo anterior, investigamos o modelo DLG através da abordagem de campo médio e obtivemos um diagrama de fases em que estão presentes três fases termodinâmicas: uma fase gasosa e duas fases líquidas, uma ordenada e outra desordenada. As três fases coexistem em um ponto triplo e a linha gás - líquido desordenado termina num ponto crítico. Neste capítulo apresentaremos o desenvolvimento de simulações de Monte Carlo pela prescrição de Metropolis do modelo DLG. Isto é necessário para verificarmos os resultados de campo médio que nem sempre preveem corretamente as fases ou a ordem das transições e também para nos prepararmos para a simulação do modelo DLG com cargas, a ser apresentado no capítulo 6. 


\subsection{Enumeração Exata}

Iniciamos nossa investigação numérica com a enumeração exata das configurações do modelo para redes pequenas. Além de permitir um estudo exploratório de propriedades do modelo, esta abordagem serve de teste para os programas desenvolvidos com base no algoritmo de Metropolis[28]. O método adotado consiste em gerar todas as configurações de uma rede pequena para obter a densidade de estados $\Omega(E, M, N)$ e, dessa forma, é calculada explicitamente a grande função de partição,

$$
\Xi(\beta, \mu, H)=\sum_{E} \sum_{M} \sum_{N} \Omega(E, M, N) \mathrm{e}^{-\beta(E-H M-\mu N)},
$$

onde $E$ é a energia da configuração, $M$ é a "magnetização" e $N$ é a quantidade de partículas, dadas respectivamente pelas equações 2.19, 2.22 e 2.23.

Nossos resultados se limitam a redes de tamanho $4 \times 4$. Neste caso a densidade de estados tem 5166 termos com valores entre 1 e $10^{48}$. É possível obter resultados de redes maiores utilizando um método recursivo, admiravelmente bem aplicado por Binder et alii [29, 30, 31], que não será utilizado neste trabalho. Enumerar redes maiores implica, além de um custo computacional maior, em trabalhar com números inteiros maiores. Para o modelo DLG e os modelos de Doniach et alii, com fator de degenerescência dos sítios no estado desordenado, isto é complicante e requer mais cuidado.

Como curiosidade, apresentamos a grande função de partição de um rede quadrada de largura 1:

$$
\Xi_{L=1}=1+e^{-\beta(-H-\mu-7.8 J)}+1000 e^{-\beta(H-\mu-0.6 J)}
$$


e de largura 2:

$$
\begin{aligned}
\Xi_{L=2}= & 1+ \\
& 10^{12} e^{-\beta(4 H-4 \mu-2.4 J)}+4 \cdot 10^{9} e^{-\beta(3 H-3 \mu-1.2 J)}+4 \cdot 10^{9} e^{-\beta(2 H-4 \mu-1.6 J)}+ \\
& 4 \cdot 10^{6} e^{-\beta(2 H-2 \mu-0.6 J)}+8 \cdot 10^{6} e^{-\beta(H-3 \mu-0.8 J)}+4 \cdot 10^{6} e^{-\beta(-4 \mu-8.8 J)}+ \\
& 2 \cdot 10^{6} e^{-\beta(2 H-2 \mu)}+4000 e^{-\beta(H-\mu)}+4000 e^{-\beta(-2 \mu)}+ \\
& 4 \cdot 10^{6} e^{-\beta(H-3 \mu-0.4 J)}+8000 e^{-\beta(-2 \mu-0.2 J)}+8000 e^{-\beta(-H-3 \mu-8 J)}+ \\
& 2 \cdot 10^{6} e^{-\beta(-4 \mu-0.8 J)}+4000 e^{-\beta(-H-3 \mu-0.4 J)}+4000 e^{-\beta(-2 H-4 \mu-16 J)}+ \\
& 4 e^{-\beta(-H-\mu)}+4 e^{-\beta(-2 H-2 \mu-7.8 J)}+2 e^{-\beta(-2 H-2 \mu)}+ \\
& 4 e^{-\beta(-3 H-3 \mu-15.6 J)}+e^{-\beta(-4 H-4 \mu-31.2 J)}
\end{aligned}
$$

sob condições periódicas de contorno para os seguintes parâmetros: fator de degenerescência $\Omega=1000, \Delta / J=0,9$ e $K / J=1,1$.

Na figura 4.1, comparamos resultados de enumeração exata com campo médio, com um resultado antecipado de simulação numérica no ensemble grande canônico. Observa-se que mesmo sistemas pequenos sugerem a existência de uma transição de fase, e para $\mu / J=-7,2, \Delta / J=0,9, K / J=1,1$ e $\Omega=1000$, a temperatura de transição é praticamente a mesma para as duas abordagens, campo médio e enumeração, exata ou incompleta. 


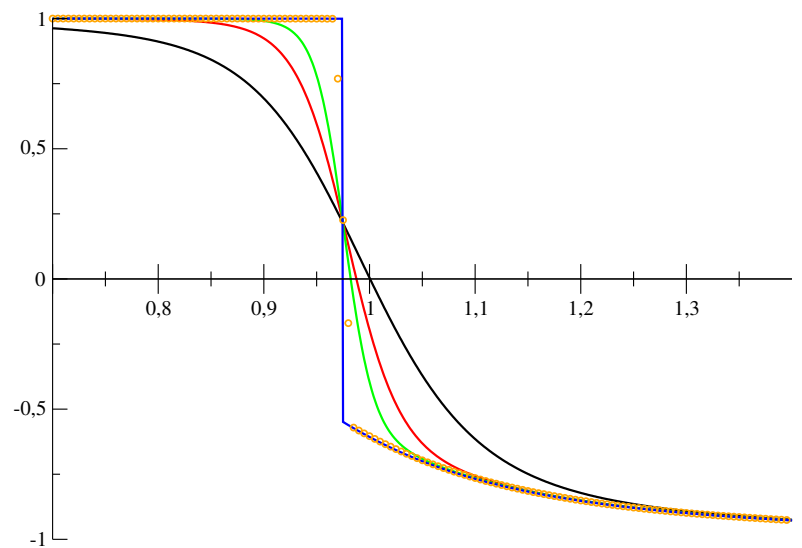

(a)

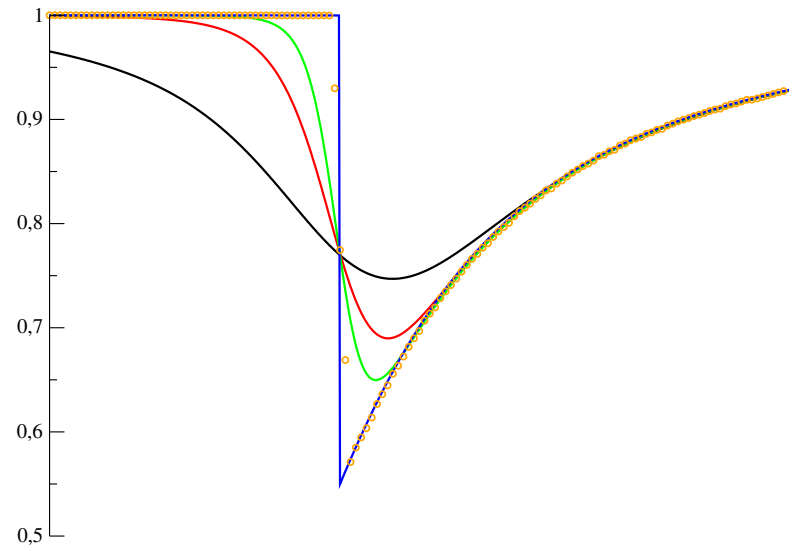

(b)

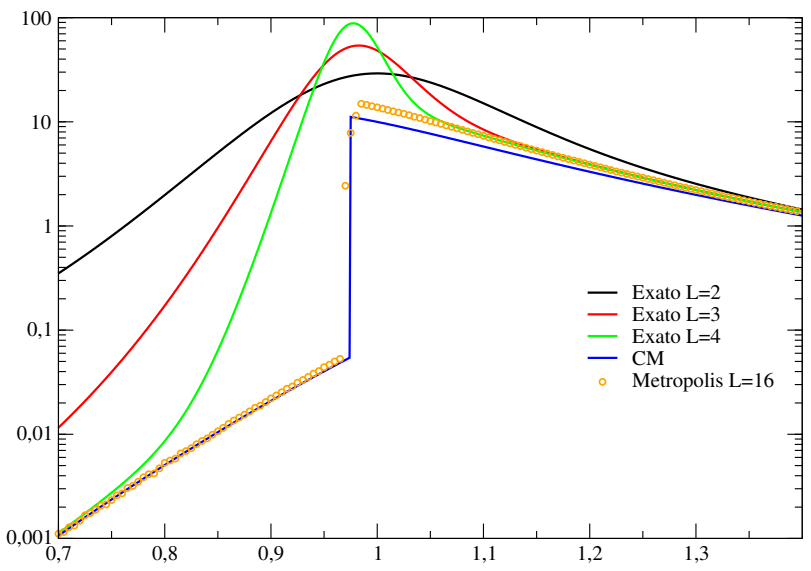

(c)

Figura 4.1: "Magnetização" (a), densidade (b) e calor específico (c) em função da temperatura, obtidas por enumeração exata para redes de largura $L=\{2,3,4\}$, campo médio $(C M)$ e por Metropolis para potencial químico $\mu / J=-7,2, \Delta / J=0,9, K / J=1,1$ e $\Omega=1000$. As simulações foram realizadas em uma rede quadrada de lado $L=16 \mathrm{com}$ condições periódicas de contorno e cada ponto representa uma média sobre vinte amostras, sendo que cada amostra consiste de cem mil passos de Monte Carlo após o mesmo período de termalização. Os resultados de calor específico para as simulações de Monte Carlo foram obtidos através do teorema dissipação - flutuação. 


\subsection{Método de Metropolis na presença de es- tados degenerados}

No método de Metropolis [32, 33, 34, 35], as configurações, ou estados microscópicos, são gerados sequencialmente a partir da configuração anterior, de acordo com uma probabilidade de transição que depende da diferença de energia entre as configurações, a temperatura do banho térmico e também dos campos termodinâmicos que existirem.

O método gera configurações estacionárias de um sistema vinculado a um reservatório térmico e, no caso do ensemble grande canônico, também a um banho de partículas. Satisfazendo a condição de balanço, é garantido que a configuração gerada a partir de uma configuração estacionária também é uma configuração estacionária. Em modelos com estados degenerados, de sítio ou de partícula, como no caso de nossas cadeias lipídicas no estado desordenado, é vantajoso definir taxas de transição diferentes das taxas propostas no artigo original de Metropolis [32]. O método consiste em escolher aleatoriamente um sítio, ou partícula, ou um grupo de sítios ou partículas, e selecionar aleatoriamente um novo estado. A chance do novo estado ser escolhido é função do algoritmo aplicado, da temperatura do banho térmico e, no caso do grande canônico, do potencial químico deste banho.

\section{Balanço detalhado}

Seja $P_{n}$ a probabilidade do estado microscópico $n$ e $W_{n \rightarrow m}$ a taxa de transição do estado $n$ para um novo estado $m$. A condição de balanço detalhado, uma 
condição particular de balanço, é dada por

$$
P_{n} W_{n \rightarrow m}=P_{m} W_{m \rightarrow n}
$$

A probabilidade do estado $n$ no ensemble canônico é dada por

$$
P_{n}=\frac{\mathrm{e}^{\frac{-E_{n}}{k_{B} T}}}{Z}
$$

e no ensemble grande canônico

$$
P_{n}=\frac{\mathrm{e}^{\frac{-\phi_{n}}{k_{B} T}}}{\Xi}
$$

onde $E_{n}$ é a energia do estado $n, \phi_{n}=E_{n}+\mu N_{n}, N_{n}$ é a quantidade de partículas do estado $n, k_{B}$ a constante de Boltzmann, $T$ a temperatura, $\mu$ o potencial químico, $Z$ a função de partição e $\Xi$ a grande função de partição. Devido a pequena diferença do procedimento seguinte neste dois ensembles, vamos apresentar o procedimento no ensemble grande canônico.

O novo estado, $m$, é escolhido entre os possíveis novos estados. Os termos da condição de balanço detalhado, eq. 4.4, podem ser rearranjados,

$$
\frac{W_{n \rightarrow m}}{W_{m \rightarrow n}}=\mathrm{e}^{\frac{-\Delta \phi}{k_{B} T}}
$$

Dessa forma a dependência com a função de partição é descartada e apenas as diferença $\Delta \phi=\phi_{m}-\phi_{n}$, o potencial químico e a temperatura regem a evolução do sistema.

A escolha da taxa de transição é arbitrária e Metropolis et al. [32] esco- 
lheram,

$$
W_{n \rightarrow m}=\min \left\{1, \mathrm{e}^{-\frac{\Delta \phi}{k_{B} T}}\right\} \quad,
$$

que significa que se a energia do estado $m$ for maior que a energia do estado $n$, então

$$
\begin{aligned}
& W_{n \rightarrow m}=\mathrm{e}^{-\frac{\Delta \phi}{k_{B} T}} \\
& W_{m \rightarrow n}=1,
\end{aligned}
$$

senão,

$$
\begin{aligned}
& W_{n \rightarrow m}=1 \\
& W_{m \rightarrow n}=\mathrm{e}^{\frac{\Delta \phi}{k_{B} T}} .
\end{aligned}
$$

Observe que esta taxa de transição depende apenas da energia, do potencial químico e da temperatura.

\section{Balanço detalhado para estados degenerados}

No caso de sistemas com estados degenerados, é conveniente modificar as taxas de Metropolis. A figura 4.2.A ilustra uma representação das possíveis transições no modelo DLG que resultam em alterar o estado de um único sítio do sistema. Existem $\Omega+1$ possíveis novos estados para um sítio em um particular estado. Entretanto, todos os $\Omega$ estados desordenados $(\sigma=-1)$ são degenerados neste modelo simplificado. Para evitar que o método passeie inutilmente entre os $\Omega$ estados desordenados degenerados, vamos representar todos os estados desordenados do sítio por um único estado desordenado 

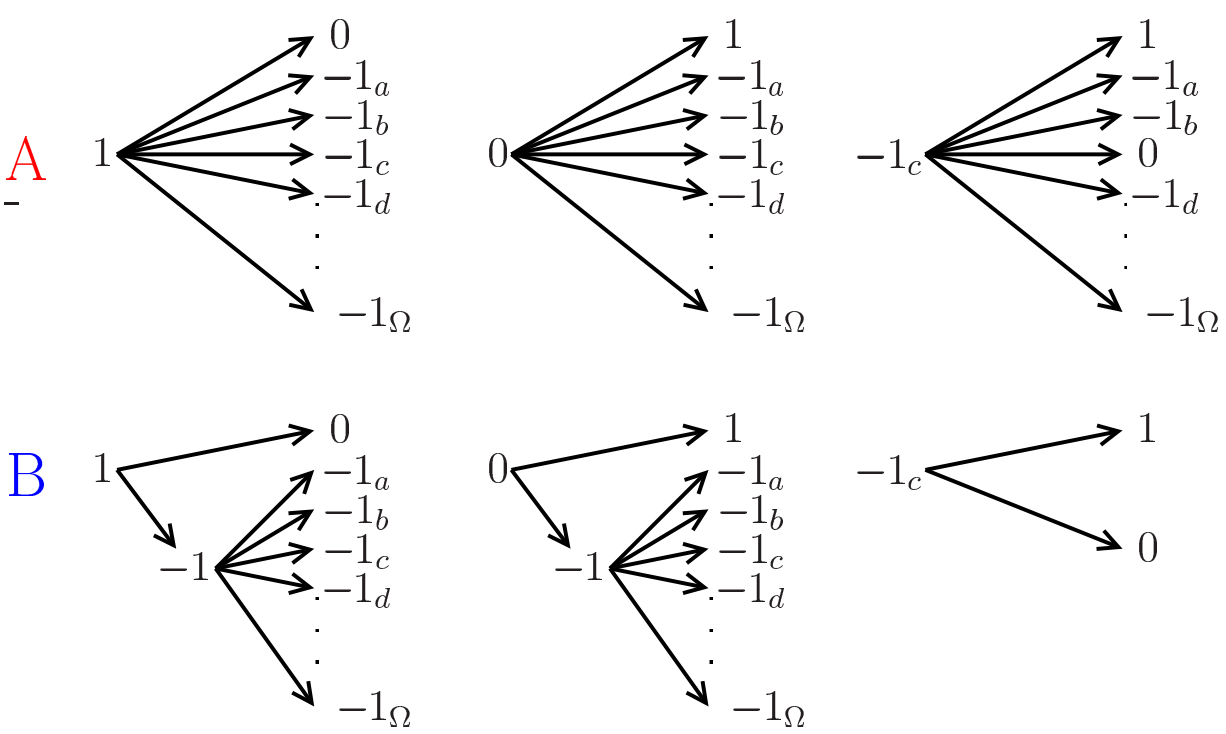

Figura 4.2: Representação da maneira tradicional (A) de escolher um novo estado e a maneira que leva em conta a degenerescência do estado desordenado (B).

com multiplicidade $\Omega$, figura 4.2.B. Neste caso, devemos lembrar que a taxa de transição $W_{n \rightarrow m}$ é o produto da taxa de aceite de transição $\Pi(n \rightarrow m)$ pela probabilidade de escolher esta transição $\alpha(n \rightarrow m)$ [33]. ${ }^{1}$ Então, por exemplo, a condição de balanço detalhado para a transição de um sítio no estado ordenado, $\sigma=1$, para algum estado fluido, $\sigma=-1$, é

$$
P(1) \overbrace{\frac{1}{\alpha} \frac{1}{\Omega}}^{\frac{1}{\Omega(1 \rightarrow-1}} \Pi\left(1 \rightarrow-1_{a}\right)=P\left(-1_{a}\right) \overbrace{\frac{1}{\alpha\left(-1_{a} \rightarrow 1\right)}}^{\frac{1}{\Omega+1}} \Pi\left(-1_{a} \rightarrow 1\right)
$$

Por isso, as taxas de transição a serem usadas nas simulações do modelo

\footnotetext{
${ }^{1}$ No artigo original de Metropolis et alii, $\alpha(n \rightarrow m)=\alpha(m \rightarrow n)$.
} 
DLG serão

$$
\begin{aligned}
W_{1 \rightarrow-1} & =\min \left\{1, \Omega \mathrm{e}^{-\frac{\Delta \phi_{1 \rightarrow-1}}{k_{B} T}}\right\} \\
W_{1 \rightarrow 0} & =\min \left\{1, \mathrm{e}^{-\frac{\Delta \phi_{1 \rightarrow 0}}{k_{B} T}}\right\} \\
W_{0 \rightarrow-1} & =\min \left\{1, \Omega \mathrm{e}^{-\frac{\Delta \phi_{0 \rightarrow-1}}{k_{B} T}}\right\} .
\end{aligned}
$$

\subsection{Cálculo eficiente das diferenças de "ener- gia": $\Delta E$ e $\Delta \phi$}

Na simulação, é importante encontrar formas eficientes de calcular diferenças de energia $E$ ou de "energia" $\phi$. Para simplificar a notação, devemos reconhecer que $\Delta E=\left.\Delta \phi\right|_{\mu=0}$.

Vamos calcular a diferença de "energia", $\Delta E$ ou $\Delta \phi$, ao se mudar o valor da variável de estado de um único sítio. Iremos identificar este sítio por $\alpha$. Na esperança de tornar o cálculo mais claro, vamos nomear as somatória de acordo com o parâmetro que a precede. Sendo assim, a "energia" inicial do sistema,

$$
\phi=-J \underbrace{\sum_{i j} \sigma_{i} \sigma_{j}}_{S_{J}}-\Delta \underbrace{\sum_{i j}\left(\sigma_{i} \sigma_{j}^{2}+\sigma_{i}^{2} \sigma_{j}\right)}_{S_{\Delta}}-K \underbrace{\sum_{i j} \sigma_{i}^{2} \sigma_{j}^{2}}_{S_{K}}-\mu \underbrace{\sum_{i} \sigma_{i}^{2}}_{S_{\mu}}
$$

é reescrita como

$$
\phi=-J S_{J}-\Delta S_{\Delta}-K S_{K}-\mu S_{\mu},
$$


e a energia do novo estado, no qual apenas o sítio $\alpha$ difere do estado inicial, é identificado como

$$
\phi^{\prime}=-J S_{J}^{\prime}-\Delta S_{\Delta}^{\prime}-K S_{K}^{\prime}-\mu S_{\mu}^{\prime}
$$

A diferença de "energia" dessa mudança é

$$
\Delta \phi=-J\left(S_{J}^{\prime}-S_{J}\right)-\Delta\left(S_{\Delta}^{\prime}-S_{\Delta}\right)-K\left(S_{K}^{\prime}-S_{K}\right)-\mu\left(S_{\mu}^{\prime}-S_{\mu}\right)
$$

A estratégia geral é separar os elementos das somatórias que dependam do estado da partícula que sofre transição, $\alpha$. Iremos mostrar o cálculo de cada grupo separadamente.

Calculo de $S_{\mu}^{\prime}-S_{\mu}$

Evidenciando $\alpha$, temos:

$$
\begin{aligned}
S_{\mu} & =\sum_{i} \sigma_{i}^{2} \\
& =\sum_{i \neq \alpha} \sigma_{i}^{2}+\sigma_{\alpha}^{2} .
\end{aligned}
$$

O mesmo é feito com $S_{\mu}^{\prime}$. Com isso temos:

$$
\begin{aligned}
S_{\mu}^{\prime}-S_{\mu} & =\sum_{i \neq \alpha} \sigma_{i}^{2}+\left(\sigma_{\alpha}^{2}\right)^{\prime}-\sum_{i \neq \alpha} \sigma_{i}^{2}-\sigma_{\alpha}^{2} \\
& =\left(\sigma_{\alpha}^{2}\right)^{\prime}-\sigma_{\alpha}^{2}
\end{aligned}
$$


Cálculo de $S_{K}^{\prime}-S_{K}$ e $S_{J}^{\prime}-S_{J}$

Dado que

$$
S_{J}=\sum_{(i j)} \sigma_{i} \sigma_{j}
$$

e identificando os vizinhos do sítio $\alpha$ como $\alpha+1, \alpha+2, \alpha+3$ e $\alpha+4, S_{J}$ é reescrito de maneira tal que é a somatória não inclua $\alpha$ :

$$
\begin{aligned}
S_{J}= & \sum_{\substack{(i j) \\
i \neq \alpha \\
j \neq \alpha}} \sigma_{i} \sigma_{j}+\frac{1}{2} \underbrace{\left(\sigma_{\alpha} \sigma_{\alpha+1}+\sigma_{\alpha} \sigma_{\alpha+2}+\sigma_{\alpha} \sigma_{\alpha+3}+\sigma_{\alpha} \sigma_{\alpha+4}\right)}_{i=\alpha} \\
& +\frac{1}{2} \underbrace{\left(\sigma_{\alpha+1} \sigma_{\alpha}+\sigma_{\alpha+2} \sigma_{\alpha}+\sigma_{\alpha+3} \sigma_{\alpha}+\sigma_{\alpha+4} \sigma_{\alpha}\right)}_{j=\alpha} \\
& =\sum_{\substack{i j) \\
i \neq \alpha \\
j \neq \alpha}} \sigma_{i} \sigma_{j}+\sigma_{\alpha}\left(\sigma_{\alpha+1}+\sigma_{\alpha+2}+\sigma_{\alpha+3}+\sigma_{\alpha+4}\right)
\end{aligned}
$$

O mesmo é feito com $S_{j}^{\prime}$, obtendo assim:

$$
\begin{aligned}
S_{J}-S_{J}^{\prime}= & \sum_{\substack{(i j) \\
i \neq \alpha \\
j \neq \alpha}} \sigma_{i} \sigma_{j}+\sigma_{\alpha}\left(\sigma_{\alpha+1}+\sigma_{\alpha+2}+\sigma_{\alpha+3}+\sigma_{\alpha+4}\right) \\
- & \sum_{\substack{(i j) \\
i \neq \alpha \\
j \neq \alpha}} \sigma_{i} \sigma_{j}-\left(\sigma_{\alpha}\right)^{\prime}\left(\sigma_{\alpha+1}+\sigma_{\alpha+2}+\sigma_{\alpha+3}+\sigma_{\alpha+4}\right) \\
= & \left(\sigma_{\alpha}-\left(\sigma_{\alpha}\right)^{\prime}\right)\left(\sigma_{\alpha+1}+\sigma_{\alpha+2}+\sigma_{\alpha+3}+\sigma_{\alpha+4}\right) .
\end{aligned}
$$

De maneira análoga, temos

$$
S_{K}-S_{K}^{\prime}=\left(\sigma_{\alpha}^{2}-\left(\sigma_{\alpha}^{2}\right)^{\prime}\right)\left(\sigma_{\alpha+1}^{2}+\sigma_{\alpha+2}^{2}+\sigma_{\alpha+3}^{2}+\sigma_{\alpha+4}^{2}\right)
$$


Cálculo de $S_{\Delta}^{\prime}-S_{\Delta}$

Semelhante ao cálculo de $S_{K}^{\prime}-S_{K}$, escrevemos $S_{\Delta}$ como

$$
\begin{aligned}
S_{\Delta}= & \sum_{\substack{(i j) \\
i \neq \alpha \\
j \neq \alpha}}\left(\sigma_{i} \sigma_{j}^{2}+\sigma_{i}^{2} \sigma_{j}\right) \\
& +\frac{1}{2} \underbrace{\left(\sigma_{\alpha} \sigma_{\alpha+1}^{2}+\sigma_{\alpha}^{2} \sigma_{\alpha+1}+\sigma_{\alpha} \sigma_{\alpha+2}^{2}+\sigma_{\alpha}^{2} \sigma_{\alpha+2}+\sigma_{\alpha} \sigma_{\alpha+3}^{2}+\sigma_{\alpha}^{2} \sigma_{\alpha+3}+\sigma_{\alpha} \sigma_{\alpha+4}^{2}+\sigma_{\alpha}^{2} \sigma_{\alpha+4}\right)}_{i=\alpha}+ \\
& +\frac{1}{2} \underbrace{\left(\sigma_{\alpha+1} \sigma_{\alpha}^{2}+\sigma_{\alpha+1}^{2} \sigma_{\alpha}+\sigma_{\alpha+2} \sigma_{\alpha}^{2}+\sigma_{\alpha+2}^{2} \sigma_{\alpha}+\sigma_{\alpha+3} \sigma_{\alpha}^{2}+\sigma_{\alpha+3}^{2} \sigma_{\alpha}+\sigma_{\alpha+4} \sigma_{\alpha}^{2}+\sigma_{\alpha+4}^{2} \sigma_{\alpha}\right)}_{j=\alpha} .
\end{aligned}
$$

Obtendo,

$$
\begin{aligned}
S_{\Delta}^{\prime}-S_{\Delta}= & \left(\left(\sigma_{\alpha}^{2}\right)^{\prime}-\sigma_{\alpha}^{2}\right)\left(\sigma_{\alpha+1}+\sigma_{\alpha+2}+\sigma_{\alpha+3}+\sigma_{\alpha+4}\right) \\
& +\left(\left(\sigma_{\alpha}\right)^{\prime}-\sigma_{\alpha}\right)\left(\sigma_{\alpha+1}^{2}+\sigma_{\alpha+2}^{2}+\sigma_{\alpha+3}^{2}+\sigma_{\alpha+4}^{2}\right) .
\end{aligned}
$$

Concluindo Se definirmos

$$
\begin{gathered}
d_{1}=\left(\left(\sigma_{\alpha}\right)^{\prime}-\sigma_{\alpha}\right) \\
d_{2}=\left(\left(\sigma_{\alpha}^{2}\right)^{\prime}-\sigma_{\alpha}^{2}\right), \\
v_{1}=\left(\sigma_{\alpha+1}+\sigma_{\alpha+2}+\sigma_{\alpha+3}+\sigma_{\alpha+4}\right)
\end{gathered}
$$


$\mathrm{e}$

$$
v_{2}=\left(\sigma_{\alpha+1}^{2}+\sigma_{\alpha+2}^{2}+\sigma_{\alpha+3}^{2}+\sigma_{\alpha+4}^{2}\right)
$$

a diferença de "energia" ao se mudar o estado do sítio $\alpha$ será convenientemente expressa por

$$
\Delta \phi=-J d_{1} v_{1}-\Delta\left(d_{2} v_{1}+d_{1} v_{2}\right)-K d_{2} v_{2}-\mu d_{2}
$$

\subsection{Ensemble Grande canônico}

No ensemble grande canônico, a temperatura e o potencial químico são constantes. O campo $H$, conjugado a "magnetização" também é constante, e vale zero. O sistema é descrito por variáveis de sítios, que assumem o estado ordenado, desordenado e vazio. Tanto a temperatura, como o potencial químico e o campo $H$, são campos, e não restringem os estados do sistema. Portanto, todos os sítios podem assumir qualquer um dos 3 estados possíveis.

O sistema é descrito em uma rede quadrada com condições periódicas de contorno.

\section{Histerese}

Estamos interessados nas propriedades do modelo próximo às transições de fases. As configurações estáveis, de equilíbrio, são aquelas que são mínimos globais da energia livre [36]. Nas proximidades da coexistência de fases, sob uma pequena variação de algum parâmetro, como a temperatura, um mínimo local se torna o mínimo global e vice e versa. Neste ponto há uma transição de fase de primeira ordem. 
Figura 4.3: Quantidade média de passos de Monte Carlo necessários para o sistema transitar uma vez entre estados gel e fluido, ou vice e versa, em função da largura da caixa de simulação. Os parâmetros são $t=1, \mu / J=-7,2, \Delta / J=0,9 \mathrm{e}$ $K / J=1,1$

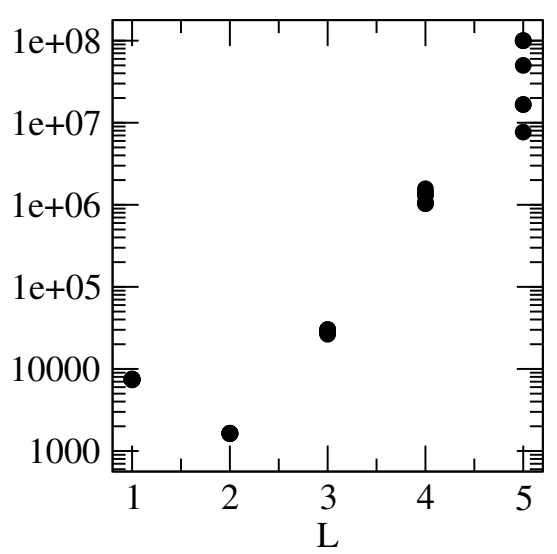

O método de Metropolis gera estados estacionários a partir de estados estacionários. Estados estacionários correspondem a mínimos da energia livre. Para o método gerar uma boa estatística perto da transição de fase, é preciso que o método permita o trânsito entre as configurações dos dois mínimos. E isto deve acontecer muitas vezes, para eliminar a histerese e obter uma linha continua que se aproxime do patamar que representa a coexistência de fases.

Conforme se aumenta o tamanho do sistema, a quantidade de configurações que descreve uma fase termodinâmica é mais rica. Por outro lado, a quantidade de configurações que separam os mínimos da energia livre também é maior. A quantidade de tentativas para que o algoritmo de Metropolis transite de um mínimo a outro é maior. Neste modelo, como é apresentado na figura 4.3, a quantidade de passos de Monte Carlo para o sistema transitar entre os estados ordenado e desordenado é impraticável.

Dada a inviabilidade de se ter uma boa estatística no ponto, é comum adotar para a coexistência o ponto médio da histerese. A histerese é gerada a partir de muitas configurações iniciais aleatórias ou, alternativamente, a partir do "esfriamento" e "aquecimento" do sistema, ilustrada na figura 4.4, 


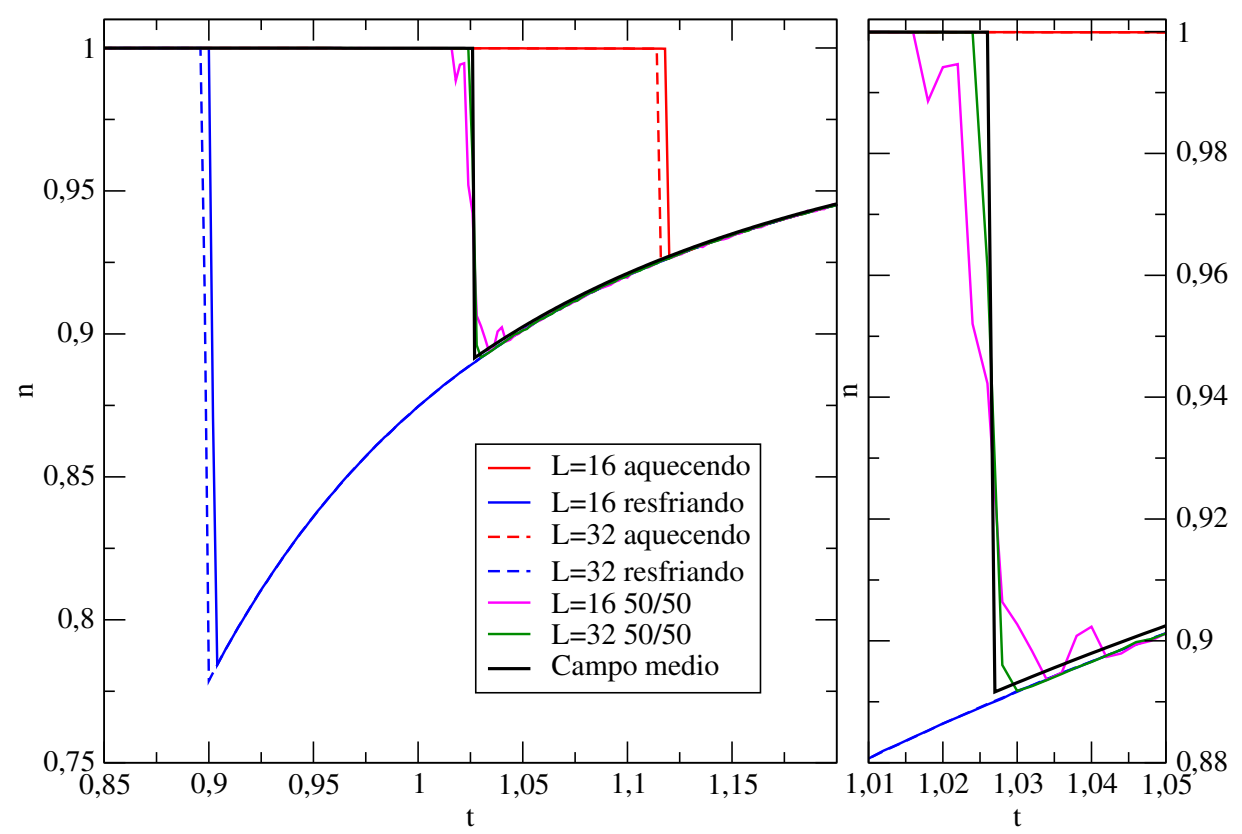

Figura 4.4: Densidade em função da temperatura, obtida pela abordagem de campo médio e por simulações pelo método de Metropolis. Os parâmetros comuns são $\mu / J=-6$, $\Delta / J=0,9$ e $K / J=1,1$. A figura da direita é um detalhe da figura da esquerda. As simulações foram divididas em dois grupos: No primeiro grupo o sistema foi aquecendo e resfriado. Cada ponto representa uma média sobre dez milhões de passos de Monte Carlo e a configuração inicial de cada ponto é a configuração final do ponto anterior; No segundo grupo, a condição inicial é sempre com metade dos sítios no estado ordenado, e metade no estado desordenado, formando dois aglomerados (50/50). Cada ponto é uma média sobre vinte simulações independentes de duzentos mil passos de Monte Carlo, com duzentos mil passos de Monte Carlo de termalização.

Na tentativa de obter configurações de equilíbrio próximo à transição de primeira ordem, apesar da existência da histerese, evoluímos o sistema a partir de uma configuração inicial na qual metade dos sítios está formando um aglomerado no estado ordenado, e metade no estado desordenado. A ideia é deixar os dois aglomerados competirem, algo parecido com nucleação. Fazer dessa forma foi inspirado nos artigos de Lipowski [37]. Na figura 4.4, comparamos este procedimento com o procedimento de aquecer e resfriar o sistema. Nas curvas das simulações com condição inicial metade ordenado 


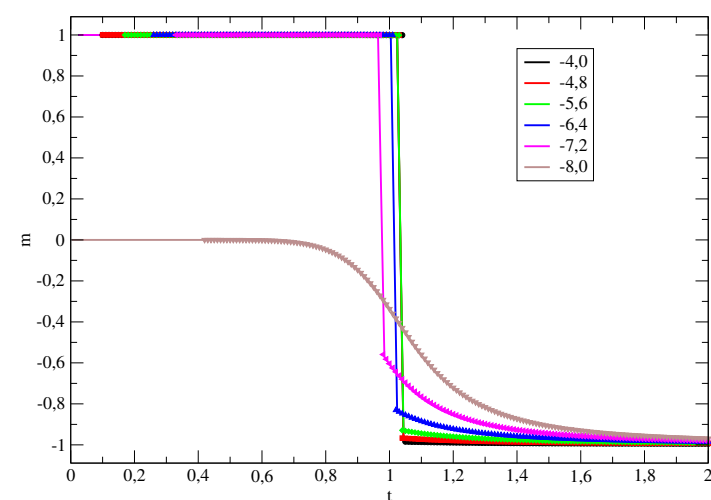

(a) "Magnetização"

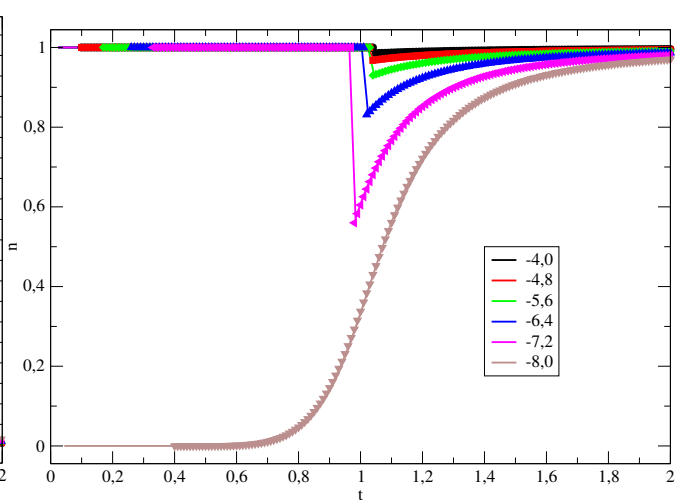

(b) Densidade

Figura 4.5: (a) "Magnetização" e (b) densidade obtidas por campo médio (-) e por Metropolis (símbolos) para $\mu / J$ de $-4,0$ a $-8,0$ e $\Delta / J=0,9$ e $K / J=1,1$. A simulações foram realizadas em uma rede quadrada de largura $L=64$ e cada ponto representa uma média sobre cem mil passos de Monte Carlo.

e metade desordenado, a histerese é bastante reduzida. Mas, mais importante, o resultado mostra que a transição está longe do ponto médio adotado em simulações com condições iniciais de aquecimento/resfriamento. Nota-se, também, uma proximidade do resultado de campo médio para os parâmetros testados, como pode ser visto nas figuras $4.5 \mathrm{a}$ e $4.5 \mathrm{~b}$. Esta proximidade sugere que o sistema está longe de um ponto crítico.

\section{Propriedades termodinâmicas}

Nas figuras 4.6a, 4.6b e 4.6c, apresentamos resultados de simulação para $K / J=1, \mu / J=-6, \Omega=1000$ e alguns valores de $\Delta / J$. As curvas de campo médio são apresentadas na mesma figura. Neste valor de potencial químico, e nesta região de parâmetros, a solução de campo médio prevê a transição ordem - desordem, cuja temperatura aumenta com $\Delta$, que é o parâmetro que estabiliza a fase ordenada, como vimos na Fig. 3.10. À 


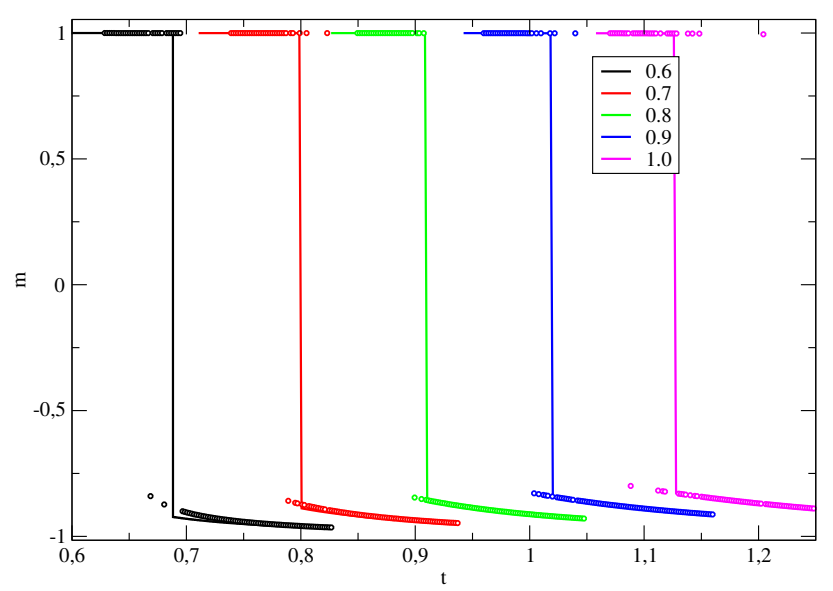

(a) "Magnetização"

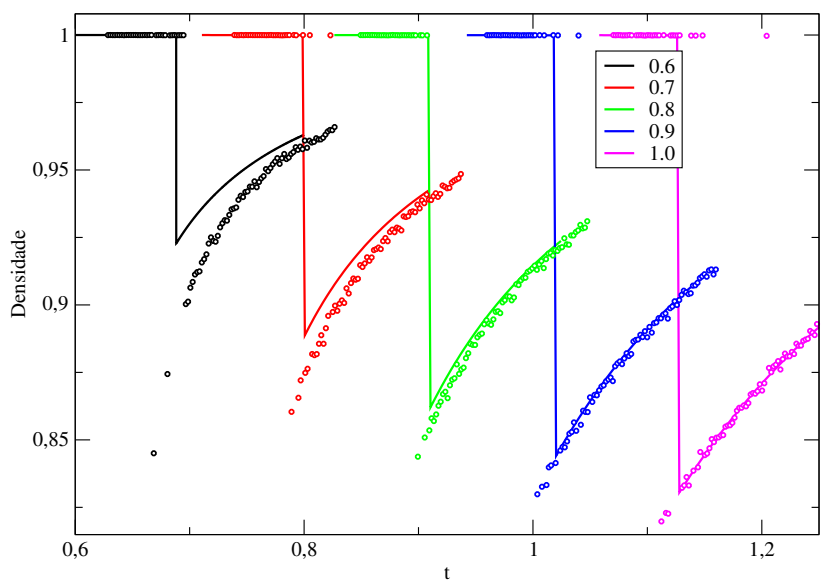

(b) Densidade

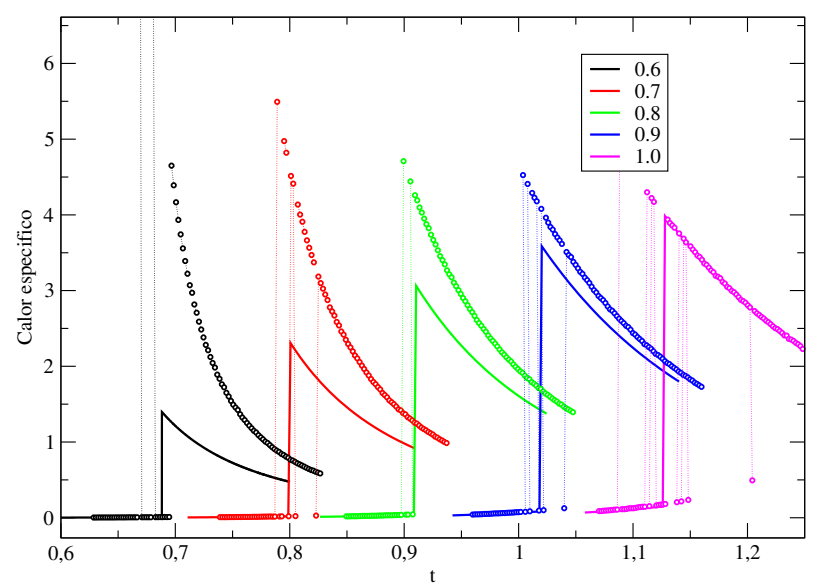

(c) Calor específico

Figura 4.6: Comparação da solução de campo médio com os resultados de Metropolis para alguns valores de $\Delta$, rede quadrada de largura $L=8$ e condição inicial com metade dos sítios no estado ordenado e metade no estado desordenado. A média é feita sobre um milhão de passos de Monte Carlo após um milhão de passos de termalização. Os parâmetros comuns são $K / J=1, \mu / J=-6$ e $\Omega / J=1000$. 
medida que $\Delta$ diminui, com potencial químico $\mu$ fixo, a descontinuidade na densidade diminui. Um exame da Fig 3.10 mostra que nos aproximamos da linha de coexistência gás - líquido desordenado, com final num ponto crítico. A proximidade de um ponto crítico pode, em princípio, explicar o distanciamento entre os resultados de campo médio e de simulações.

\subsection{Ensemble Canônico}

No ensemble canônico, a quantidade de partículas e a temperatura são constantes. As mudanças de estados envolvidas nas simulações neste ensemble devem conservar estas grandezas: adotamos a difusão dos lipídios e a troca entre os estados ordem e desordem das cadeias.

No ensemble grande canônico, a quantidade de partículas flutua. A quantidade de partículas de uma configuração qualquer, por ser uma grandeza extensiva, é determinada exatamente e, portanto, o cálculo da média é trivial.

Entretanto, o potencial químico no ensemble canônico flutua e deve ser obtido indiretamente em termos do valor médio de grandeza correlacionada. Escolhemos o método de Widom para este propósito.

\subsubsection{Método de Widom e enumeração exata}

Para se calcular o potencial químico nas simulações no ensemble canônico, utilizaremos o método de Widom [38]. O método estima a derivada da energia livre de Helmholtz pela quantidade de partículas, isto é, o potencial químico 
a temperatura e volume constantes.

$$
\mu=\left(\frac{\partial F}{\partial N}\right)_{T, V} \approx F_{N+1}-F_{N}=-k_{B} T \ln \frac{Z_{N+1}}{Z_{N}}
$$

É conveniente descrever o sistema pelas variáveis de posição $\mathbf{r}$ de cada lipídio e pelo estado $s$ de suas cadeias hidrofóbicas. Nestas varáveis, a função de partição é dada por:

$$
Z_{N}=\sum_{\mathbf{r}_{1}} \cdots \sum_{\mathbf{r}_{N}} \sum_{s_{1}} \cdots \sum_{s_{N}} \frac{1}{N !} \mathrm{e}^{-\beta E_{N}}
$$

onde $E_{N}=E_{N}\left(s_{1}, \cdots, s_{N}, \mathbf{r}_{1}, \cdots, \mathbf{r}_{N}\right)$ é a energia do estado com $N$ lipídios, definido pelo conjunto de variáveis de posição dos lipídios $\left\{\mathbf{r}_{1}, \cdots, \mathbf{r}_{N}\right\}$ e pelo conjunto de variáveis de estado das cadeias $s=\{-1,1\}$. A função de partição do estado com $N+1$ lipídios é

$$
Z_{N+1}=\sum_{\mathbf{r}_{1}} \cdots \sum_{\mathbf{r}_{N}} \sum_{\mathbf{s}_{1}} \cdots \sum_{\mathbf{s}_{N}} \sum_{\mathbf{r}_{N+1}} \sum_{\mathbf{s}_{N+1}} \frac{1}{(N+1) !} \mathrm{e}^{-\beta E_{N}} \mathrm{e}^{-\beta \Delta E}
$$

onde $\Delta E=E_{N+1}-E_{N}$. Dividindo a equação 4.38 pela equação 4.37 , temos:

$$
\frac{Z_{N+1}}{Z_{N}}=\frac{1}{N+1} \frac{\frac{1}{N !} \sum_{\mathbf{r}_{1}} \cdots \sum_{\mathbf{r}_{N}} \sum_{\mathbf{s}_{1}} \cdots \sum_{\mathbf{s}_{N}} \mathrm{e}^{-\beta E_{N}} \sum_{\mathbf{r}_{N+1}} \sum_{\mathbf{s}_{N+1}} \mathrm{e}^{-\beta \Delta E}}{\frac{1}{N !} \sum_{\mathbf{r}_{1}} \cdots \sum_{\mathbf{r}_{N}} \sum_{\mathbf{s}_{1}} \cdots \sum_{\mathbf{s}_{N}} \mathrm{e}^{-\beta E_{N}}}
$$

Por inspeção, reconhece - se que:

$$
\frac{Z_{N+1}}{Z_{N}}=\frac{1}{N+1}\left\langle\sum_{\mathbf{r}_{N+1}} \sum_{\mathbf{s}_{N+1}} \mathrm{e}^{-\beta \Delta E}\right\rangle_{N}
$$


onde $\langle\ldots\rangle_{N}$ corresponde à média no ensemble de $N$ partículas.

O método de Widom consiste das equações 4.36 e 4.40. Para verificar o método, é interessante fazer enumeração exata de sistema pequenos. É mais fácil calcular a função de partição por enumeração exata quanto nesta forma:

$$
\left.Z_{N}=\sum_{\{\sigma\}} \delta\left(N, \sum_{i} \sigma_{i}^{2}\right)\right) \mathrm{e}^{-\beta E(\{\sigma\})}
$$

onde o estado do sítio é descrito pela variável $\sigma=\{-1,0,1\}$, a soma é sobre todos os estados, porém, o delta de Kronecker $\delta(N, N(\{\sigma\}))$ faz com que os estados com quantidade de lipídios diferente de $N$ sejam descartados. O lado esquerdo da equação 4.40 pode ser obtido através do calculo de $Z_{N}$ e $Z_{N+1}$ pela equação 4.41. O lado direito 4.40 pode ser obtido com

$$
\left\langle\sum_{\mathbf{r}_{N+1}} \sum_{\mathbf{s}_{N+1}} \mathrm{e}^{-\beta \Delta E}\right\rangle_{N}=\frac{\sum_{\{\sigma\}} \delta\left(N, \sum_{i} \sigma_{i}^{2}\right) \mathrm{e}^{-\beta E_{N}} \sum_{\mathbf{r}_{N+1}} \sum_{\mathbf{s}_{N+1}} \mathrm{e}^{-\beta \Delta E}}{\sum_{\{\sigma\}} \delta(N, N(\{\sigma\})) \mathrm{e}^{-\beta E_{N}}} .
$$

Como alternativa ao método de Widom, existe o método do M. J. de Oliveira [39]. Os princípios envolvidos são os mesmos. A diferença é que em vez de adicionar virtualmente uma partícula, retira-se virtualmente uma partícula. Para economizar recursos computacionais, estes métodos não costumam ser realizados sobre toda a rede. Em vez disso, são realizados sobre uma amostra. Feito dessa maneira, o método de Widom é pouco eficiente a alta densidade e o método de de Oliveira é pouco eficiente em baixa densidade. Por simplicidade, neste trabalho escolhemos sacrificar recursos computacionais e usar principalmente o método de Widom, mas realizado sobre 


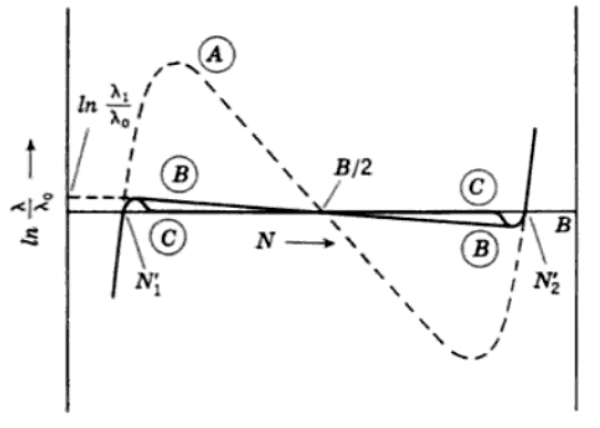

Figura 4.7: Figura esquemática das isotermas de potencial químico versus densidade. figura 73 da referência [40]

todo o sistema, para garantir a qualidade do resultado mesmo em alta densidade.

\subsubsection{Resultados da simulação no ensemble canônico}

Nas simulações do ensemble grande canônico, a coexistência de fases é sinalizada pela presença de histerese. Nas simulações do ensemble canônico, a característica que indica a coexistência de fases é a presença de "laços" nas isotermas de potencial químico [40,41]. Este fato é ilustrado na figura 4.7 para o gás de rede simples, no qual, podemos ver dois laços que ladeiam um patamar: um de convexidade negativa e outra positiva. O patamar representa o potencial químico de coexistência entre as duas fases, uma de densidade baixa e a outra de densidade alta.

\section{Propriedades termodinâmicas}

Nas figuras 4.8a e 4.8b, apresentamos algumas isotermas obtidas por enumeração exata para o modelo DLG. Nas temperaturas mais altas 4.8a, 0, 8 e 0,9, o potencial químico é bem comportado e cresce monotonicamente com a densidade. Nas temperaturas mais baixas $4.8 \mathrm{~b}$, surgem regiões de potencial 


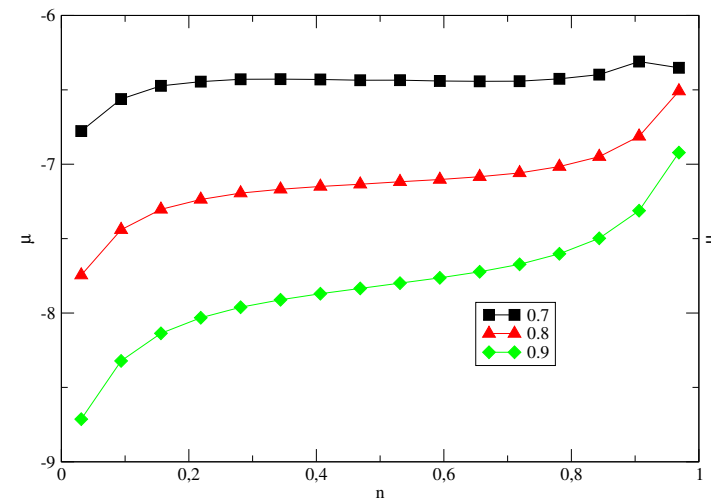

(a) Alta temperatura

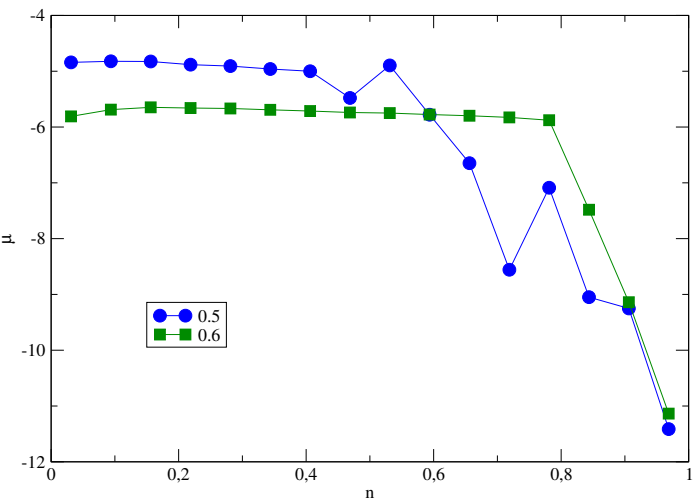

(b) Baixa temperatura

Figura 4.8: Isotermas de potencial químico $\mu$ por densidade $n$ para varias temperaturas. A direita, para temperaturas mais baixas, observa-se o decréscimo de potencial químico para densidade crescente, nas densidades altas, característico da coexistência de fases em sistemas pequenos. Resultados de enumeração exata, para rede de tamanho $4 \times 4$, os parâmetros são $\Omega=1000, \Delta / J=0,6$ e $K / J=1,1$.

químico decrescente com a densidade, de convexidade proibida no limite termodinâmico. Este efeito deve - se ao tamanho finito do sistema, e só aparece na região de coexistência de fases, correspondendo ao laço de convexidade positiva. Como foi visto, para sistemas muito pequenos não há patamar.

Os laços nas isotermas de potencial químico persistem mesmo para sistemas grandes [40, 41]. A figura 4.9 apresenta isotermas do potencial químico em função da densidade, obtidas a partir das simulações para diferentes tamanhos de rede. Na figura da esquerda, podemos ver que os laços diminuem e que o patamar de coexistência cresce, com o aumento do tamanho do sistema. Além disso, um ponto relevante é que a posição do patamar tem dependência desprezível do tamanho. Espera-se que os laços desapareçam no limite termodinâmico, mas a evolução para este limite é lenta, como pode se ver no detalhe da figura 4.9 à direita, em que o laço convexo aparece mesmo para 

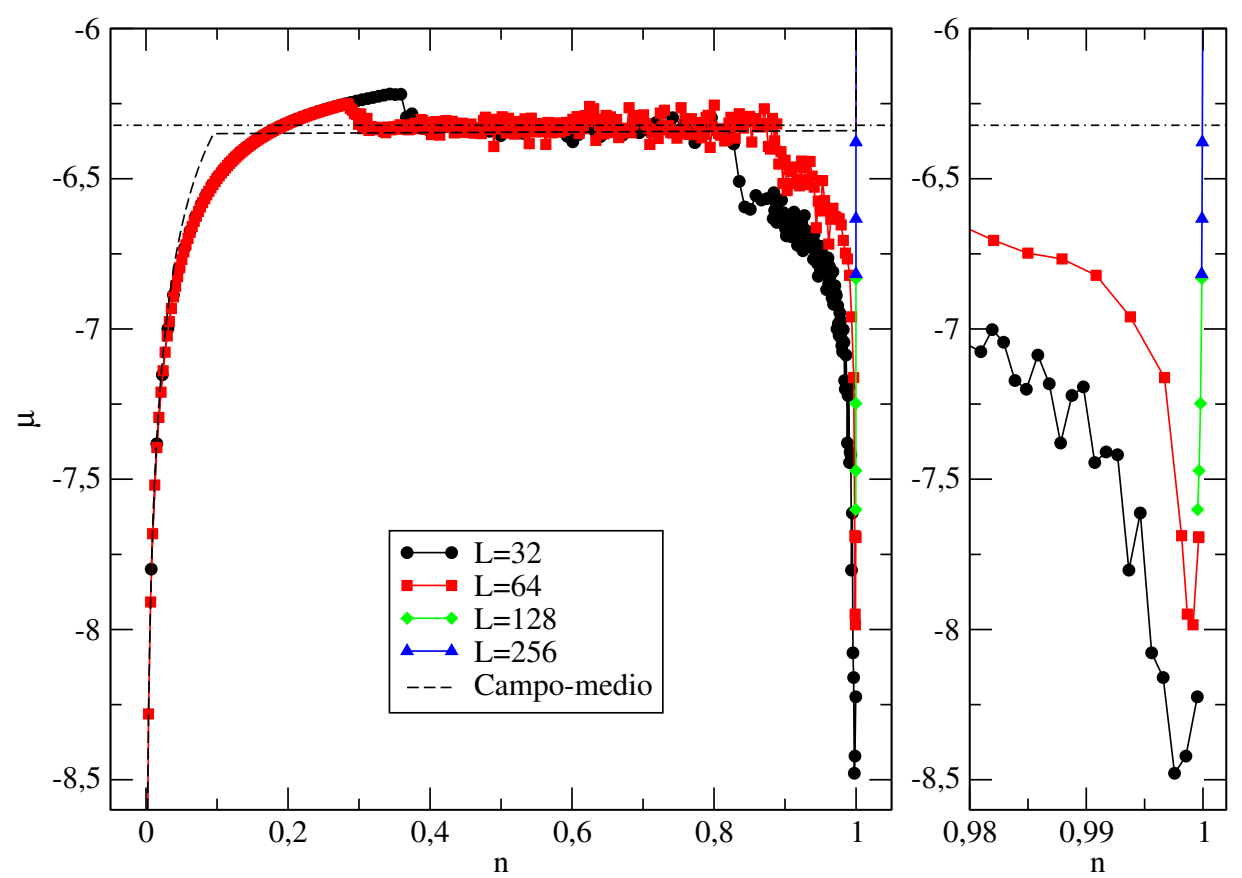

Figura 4.9: Resultados de simulação no ensemble canônico, potencial químico pela densidade em $t=0,66$. Os parâmetros são $\Omega=1000, \Delta=0,6$ e $K=1,1$. Cada ponto representa uma média sobre 3 amostras de um milhão de passos de Monte Carlo cada. A figura da direita é um detalhe da figura da esquerda para densidades altas. Pode-se observar a recuperação do potencial químico crescente com a densidade, próximo de $n=1$, que só é possível obter utilizando redes maiores.

$L=256$.

Uma questão técnica importante é que um potencial químico crescente em alta densidade só pode ser obtido para sistemas suficientemente grandes. Como o limite de densidade para o cálculo de potencial químico no método de Widom é $\frac{A-\frac{1}{2}}{A}$, para sistemas pequenos, não encontramos o potencial químico crescente próximo de densidade um, como podemos ver no detalhe à direita, para $L=32$ e $L=64$. Podemos ver também no detalhe que o único tamanho em que o potencial químico de coexistência é ultrapassado é o de $L=256$. Devido ao custo computacional, a maior parte das nossas simulações foi feita em sistemas de tamanho $L=32$, pois o patamar da coexistência, que é o 


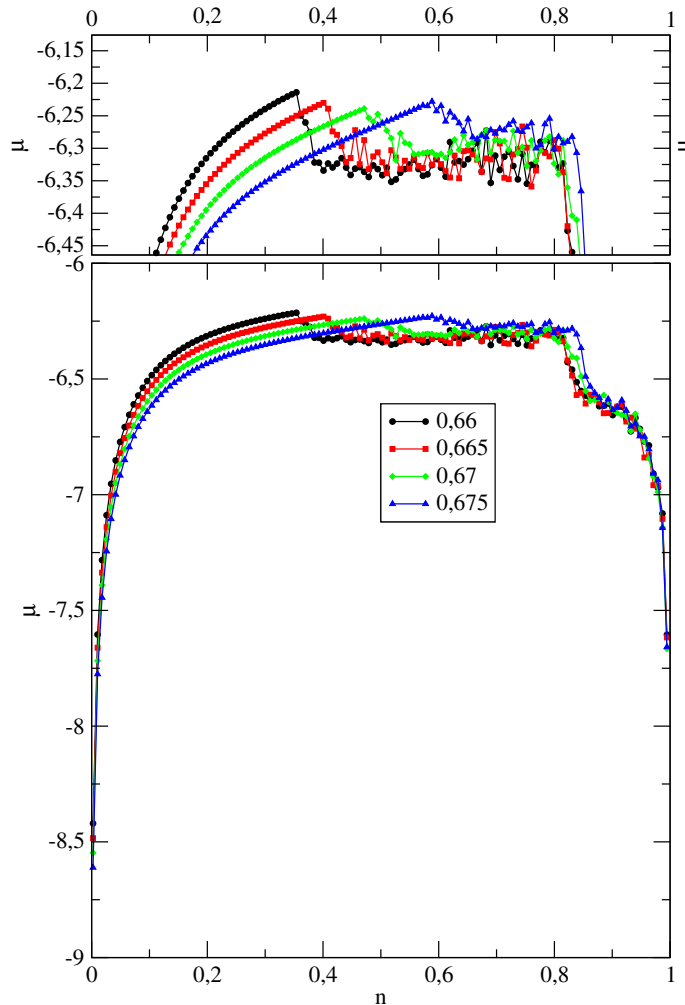

(a) Baixa temperatura

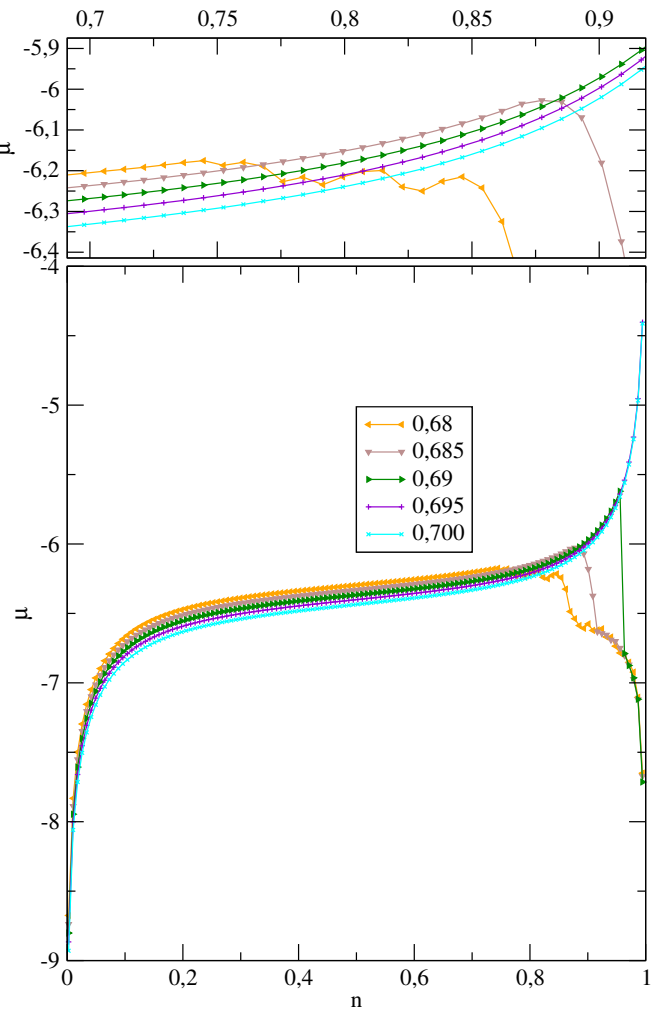

(b) Alta temperatura

Figura 4.10: Resultados de simulação no ensemble canônico em uma rede de tamanho $32 \times 32$, potencial químico pela densidade para diferentes temperaturas. Os parâmetros são $\Delta=0,6$ e $K=1,1$. Cada ponto representa uma média sobre 3 amostras de um milhão de passos de Monte Carlo cada após um milhão de passos de termalização.

que nos interessa, já é visível para este tamanho.

Na figura 4.10 apresentamos dois conjuntos de isotermas de potencial químico em função da densidade. Para temperaturas mais baixas, fig. 4.10a, os patamares de coexistência se concentram numa pequena região de potencial químico. Para as temperaturas mais altas, o patamar se desloca rapidamente para valores mais altos e desaparece na temperatura maior. Pode-se observar que o laço convexo estende-se até densidade um.

A inspeção dos patamares da figura 4.10 permite extrair dados para a 


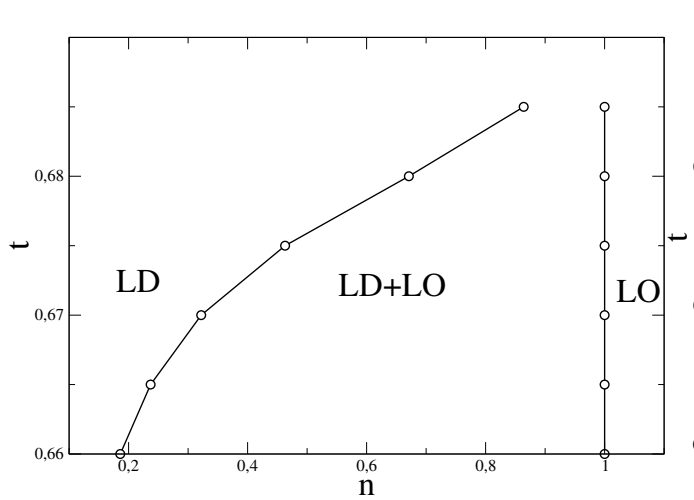

(a) Baixa temperatura

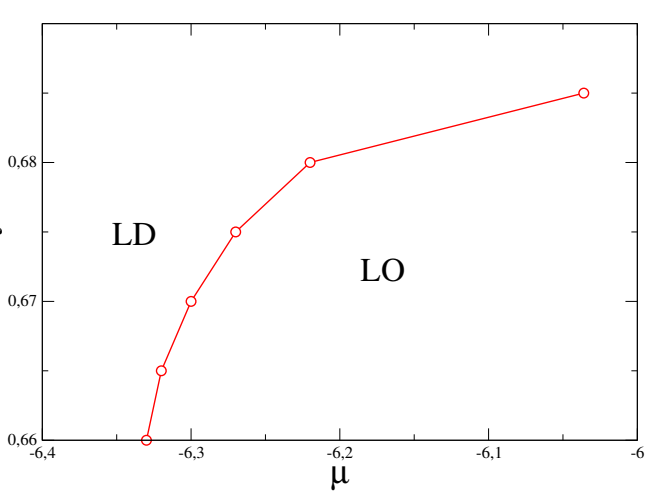

(b) Alta temperatura

Figura 4.11: Linhas de coexistência dos estados líquido ordenado (LO) e líquido desordenado (LD) nos diagramas de fases temperatura - densidade (a) e temperatura - potencial químico (b). Os parâmetros são $\Delta=0,6, K=1,1$ e $L=32$.

construção dos diagramas de fases temperatura - densidade e temperatura potencial químico, representados na figura 4.11. Em cada uma das isotermas, para obter as densidades de coexistência, estendemos o patamar à esquerda e à direita, até que este encontre as partes estáveis da isoterma. Os pontos de encontro representam as densidades de coexistência na temperatura da isoterma. Na figura 4.11a, apresentamos o resultado desse procedimento, para o diagrama de fases temperatura - densidade. As linhas de transição no diagrama de fases temperatura versus potencial químico, mostrada na figura 4.11b, são obtidas diretamente a partir dos valores do potencial químico do patamar em cada uma das isotermas, os pontos representam pontos de descontinuidade na densidade.

Na figura 4.12, examinamos o comportamento do parâmetro de ordem das cadeias, $m$. Podemos observar que nas temperaturas mais altas, 0,69 a 0,7, a "magnetização" por sítio decresce continuamente com o potencial químico de zero para valores negativos, indicando que o potencial químico enche a 


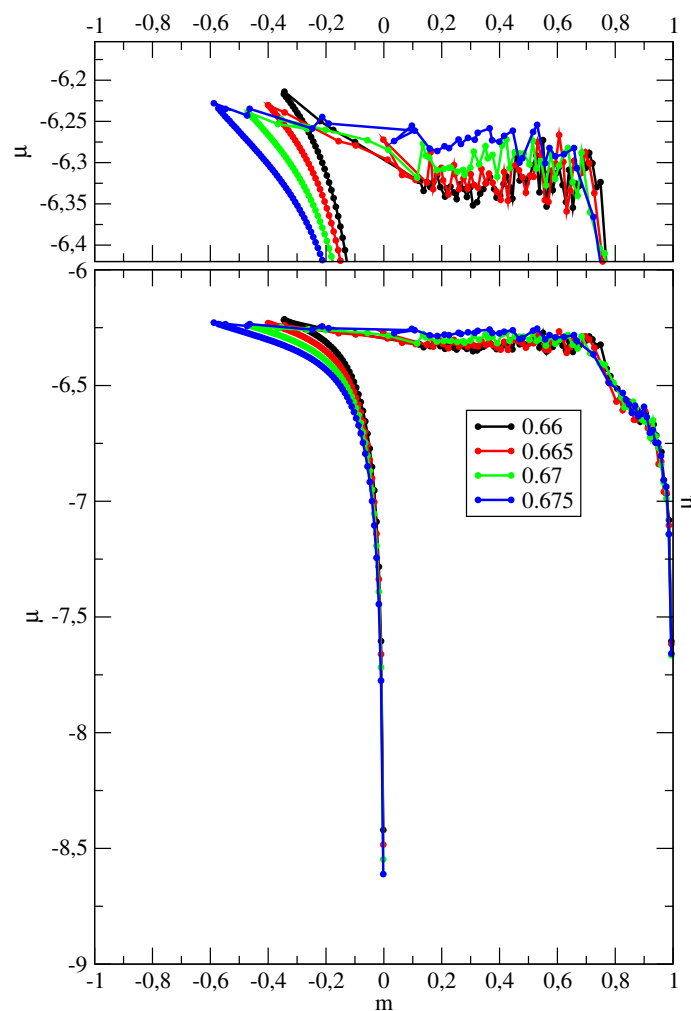

(a) Baixa temperatura

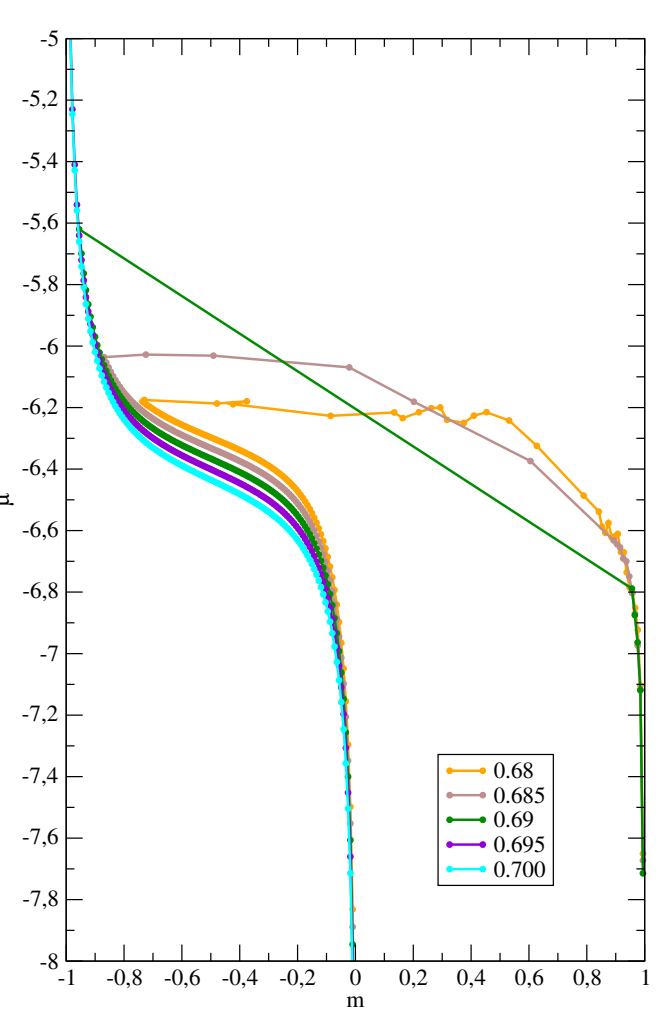

(b) Alta temperatura

Figura 4.12: Resultados de simulação no ensemble canônico em uma rede de tamanho 32x32, potencial químico pela "magnetização" para diferentes temperaturas. Os parâmetros são $\Delta / J=0,6$ e $K / J=1,1$. Cada ponto representa uma média sobre 3 amostras de um milhão de passos de Monte Carlo cada após um milhão de passos de termalização.

rede com lipídios no estado desordenado. À medida que a temperatura diminui, surge um patamar nas isotermas. Observamos que a "magnetização" por sítio, inicialmente nula, torna - se gradualmente negativa, com potencial químico crescente. Em um determinado potencial químico, onde há um patamar, o sistema transiciona abruptamente de magnetização negativa para magnetização positiva. Podemos interpretar este comportamento em termos de uma densidade crescente com o potencial químico, inicialmente de ca- 


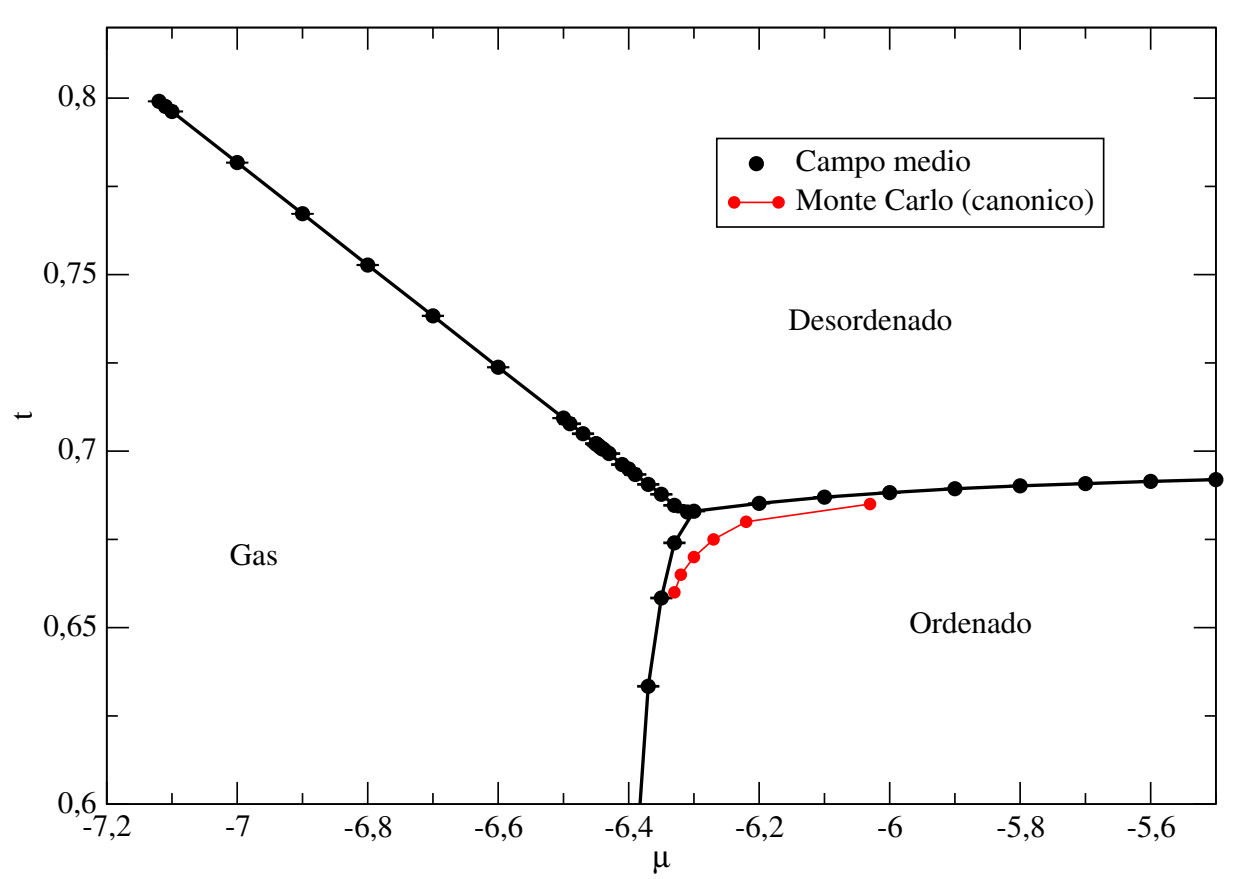

Figura 4.13: Diagrama de fase de campo médio com a inclusão dos novos resultados de simulação, obtidos com a figura 4.10. Os parâmetros são $\Delta / J=0,6$ e $K / J=1$ e $\Omega=1000$.

deias no estado desordenado, com uma transição descontínua para o estado ordenado. Assim, podemos identificar a coexistência em baixas temperaturas como uma transição gás - "líquido ordenado". As transições a temperaturas superiores poderiam ser identificadas como uma transição "líquido desordenado" - "líquido ordenado". No entanto, na região desordenada será necessário encontrar uma linha de coexistência líquido - gás, que delimitaria a fase gasosa.

Na figura 4.13, apresentamos uma comparação dos resultados de campo médio com os resultados obtidos por simulações no ensemble canônico. Na abordagem por campo médio, para este mesmo conjunto de parâmetros, observamos a transição gás - "líquido desordenado". Em nossas simulações, não observamos descontinuidade nos parâmetros de ordem, densidade e "magne- 
tização", que possa definir um interface entre estas duas fases. 


\section{Capítulo 5}

\section{Solução salina e o modelo}

\section{LRPM}

É noite e um homem está procurando alguma coisa sob a luz de um poste de iluminação pública. Um outro passa e pergunta:

- O que faz aqui?

- Procuro por minhas chaves.

O outro começa a ajudá-lo. Passado algum tempo de busca infrutífera, pergunta:

- Mas você tem certeza de que as perdeu aqui?

- Não, eu as perdi lá adiante.

- E por que procura aqui?

- Porque aqui tem luz, lá não.

A partir de uma fábula afegã, revista pesquisa fapesp, Abril, 2012.

Neste capítulo iremos rever algumas ferramentas existentes para simular sistemas carregados simples. A interação Coulombiana é de longo alcance e 
por isso deve-se ter mais cuidado com as condições de contorno, que serão discutidas no decorrer do capítulo e que devem ser escolhidas pensando no problema físico a ser mimetizado. Isto está relacionado com o cálculo do potencial Coulombiano, necessário para implementar os diversos métodos de simulação numérica, em especial, o método de Metropolis. Existem alguns métodos para realizar este cálculo, como o método rápido de multipolos[42], o método de Ewald e suas variantes[33, 34, 43, 44].

O método de Ewald só é valido para sistemas isotrópicos. Como nosso sistema de interesse é anisotrópico, desenvolvemos o cálculo direto do potencial Coulombiano e comparamos os resultados com os resultados obtidos por Ewald, com o intuito de verificar possíveis limitações do cálculo direto (para um sistema isotrópico).

Além disso, será apresentada uma técnica exata, aparentemente inédita para sistemas carregados, que calcula a diferença de energia entre estados. Aplicamos esta técnica ao método de Ewald e ao cálculo direto, diminuindo o custo computacional.

\subsection{Definição do modelo LRPM}

Iremos usar como cobaia a versão de gás de rede do RPM, o modelo primitivo restrito na rede (LRPM). O modelo primitivo restrito (RPM) representa soluções iônicas neutras de esferas duras carregadas, a saber, cátions e ânions, todas com carga de igual magnitude e mesmo diâmetro. O primeiro trabalho notório sobre este modelo foi feito por Debye e Hückel em 1923 [25, 45].

O LRPM é definido em uma rede cúbica, na qual as interações são da- 
das pela restrição de volume excluído fornecido pela rede e pelo potencial Coulombiano,

$$
\phi_{i j}=\frac{q_{i} q_{j}}{r_{i j}},
$$

onde $r_{i j}=\left|\mathbf{r}_{i}-\mathbf{r}_{j}\right|$ é a distância entre os íons $i$ e $j$, e a carga dos íons pode assumir valores $q_{i}=+1 \mathrm{ou}-1$. As partículas estão restritas à caixa de simulação a fim de conservar a densidade do sistema. A largura do sítio representa o diâmetro dos íons.

\subsection{Convergência da energia eletrostática}

Sistemas com interação de longo alcance são aqueles nos quais a energia potencial de pares tem decaimento mais lento que $|r|^{-d-\epsilon}$, para $|r|$ suficientemente grande, sendo que $d$ é a dimensão do sistema e $\epsilon$ um número infinitesimal positivo. O potencial Coulombiano vai com $|r|^{-1}$, sendo assim, esta condição é satisfeita em duas ou mais dimensões.

Ao contrário dos sistemas de interação de curto alcance, a distribuição em equilíbrio com sistemas de interação de longo alcance deve ser bastante influenciada pelos detalhes das condições de contorno [33, 34, 46, 47, 48, 49, $50,51,52]$.

Como exemplo, vamos imaginar uma distribuição suficientemente homogênea de partículas em um sistema com $d$ dimensões. A quantidade média de partículas a distância $r$ de uma partícula de referência qualquer é proporcional a $r^{d-1}$. Vamos supor que a energia de interação entre as partículas seja proporcional a $r^{-l}$, dessa forma a energia associada a uma partícula é 
proporcional a

$$
E \propto \int_{a}^{R} \frac{1}{r^{l}} r^{d-1} \mathrm{~d} r \propto \frac{R^{d-l}-a^{d-l}}{d-l} \quad d \geq 1 \quad \text { e } \quad d \neq l
$$

onde $a$ é a menor distância entre partículas.

Se $l>d$, a interação é de curto alcance. O tamanho do sistema, $R$, é tipicamente muito maior que $a$ e, portanto, o termo $R^{d-l}$ vai ser desprezível perante o termo $a^{d-l}$ e a energia de uma partícula independe do tamanho do sistema, $R$. No limite termodinâmico, $R \rightarrow \infty, R^{d-l}$ vai a zero.

Se $d>l$, a interação é de longo alcance, o termo $R^{d-l}$ predomina sobre o termo $a^{d-l}$, e a energia associada a uma partícula é função do tamanho do sistema, $R$. A contribuição das interações distantes para a energia é tão ou mais relevante que a contribuição das interações próximas. Em outras palavras, a energia devido à superfície domina a energia devido ao bulk. No limite termodinâmico em, que $R \rightarrow \infty$, a energia total por volume ou por partícula diverge.

Experimentalmente não se reconhece alterações nas propriedades termodinâmicas de soluções salinas devido à mudança da forma e/ou tamanho do recipiente. Então deve haver algum mecanismo natural que suprime os efeitos de borda, o que motiva a inclusão de condições de contorno que tentam reproduzir este mecanismo. 


\subsection{Condições de contorno}

Existem várias maneiras de impor condições de contorno, e talvez a mais simples seja impedir a passagem de partículas através da borda da caixa de simulação, representada na figura 5.1a. Vamos discutir separadamente as condições de contorno aplicadas em sistemas de curto e longo alcance.

\section{Curto alcance}

Em sistemas com interação de curto alcance, podemos separar a energia total da caixa de simulação em uma parte que escala com o volume da caixa e outra que escala com a área da superfície. No limite termodinâmico, a parte que escala com a área é desprezível perante a parte que escala com o volume, denominada bulk. Simulações computacionais são limitadas a uma quantidade pequena de partículas que pode não ser suficiente para representar um sistema no qual a energia devido às bordas seja desprezível perante a energia total.

Em sistemas pequenos, a estratégia típica consiste em impor condições de contorno artificiais, que fazem a caixa de simulação parecer fazer parte do interior de um sistema imenso, com as mesmas características do sistema contido na caixa de simulação. Dessa forma, não haverá mudança de meio ao se cruzar a fronteira da caixa de simulação.

Dentro da tradição da física da matéria condensada, existem as condições periódicas de contorno, amplamente usadas em simulações computacionais e modelos matemáticos. Neste contexto, a caixa de simulação equivale a uma célula unitária de um cristal cujo estado varia com o tempo. A figura 

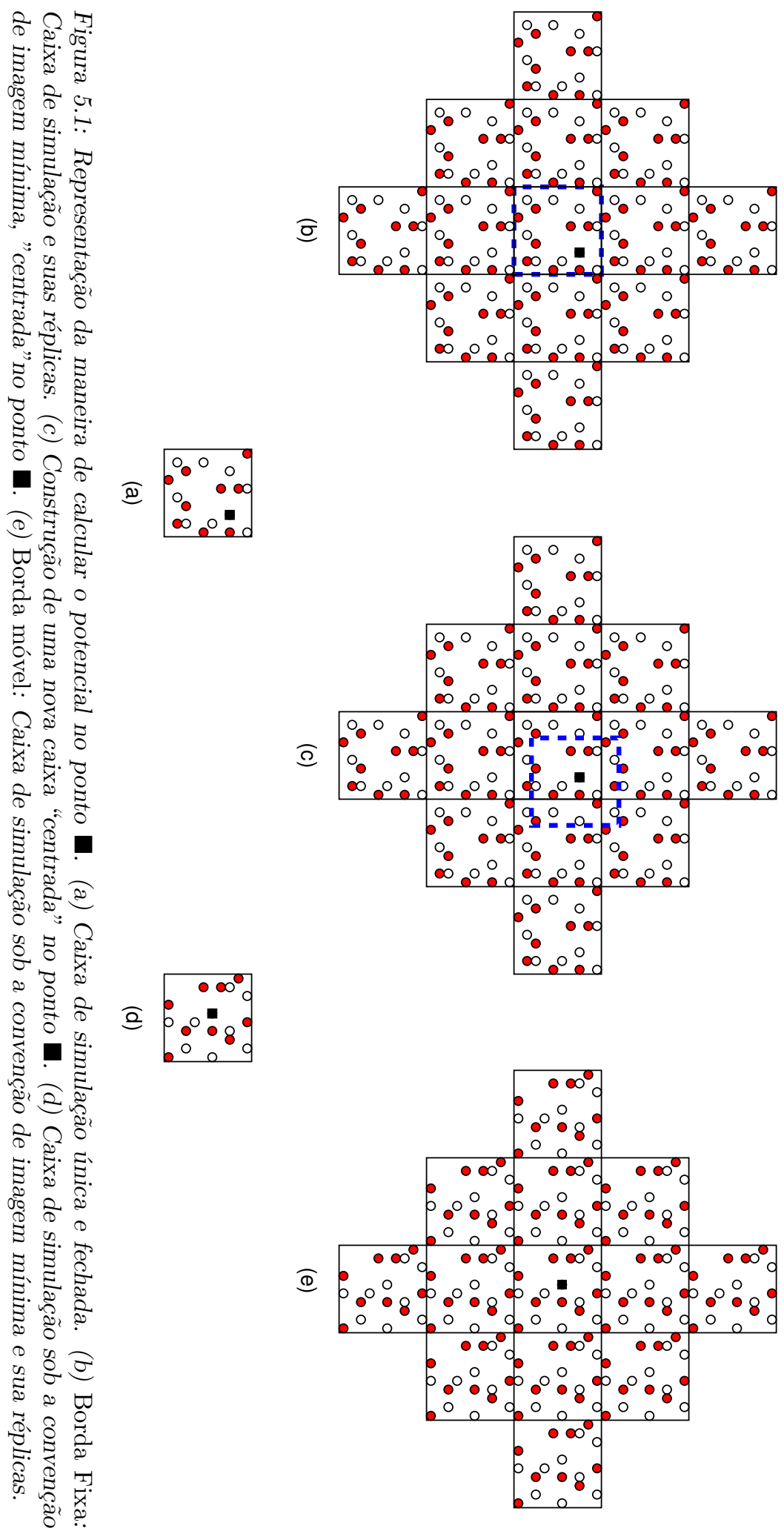

อ
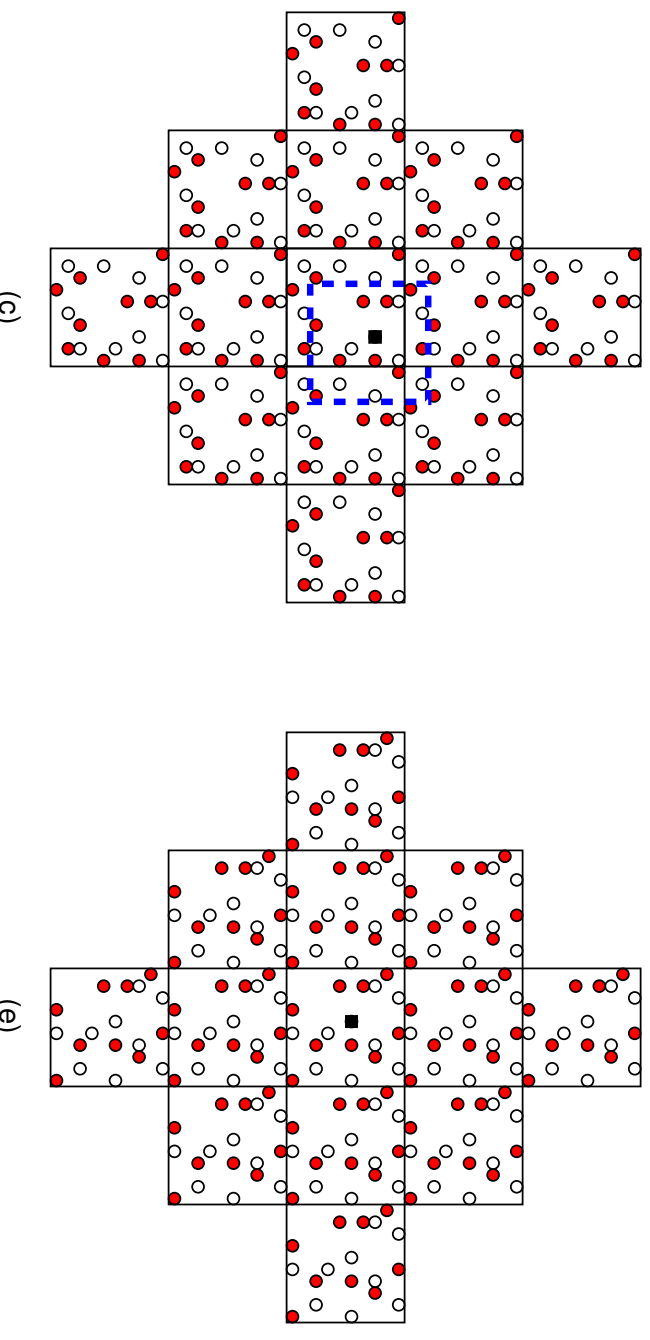
$5.1 b$ representa uma configuração desse cristal com a caixa de simulação no centro.

Nas simulações com interação de curto alcance utiliza-se frequentemente o conceito de imagem mínima[32]. A figura 5.1c ilustra a construção da nova caixa de simulação sob a convenção de imagem mínima, figura 5.1d. Na convenção de imagem mínima, a caixa de simulação é redefinida de maneira tal que a partícula em foco está situada no centro da nova caixa de simulação, ou no caso de um reticulado de lado par, próximo do centro. Isto é similar a um raio de corte no formato da caixa de simulação. Em um sistema bidimensional de curto alcance, como do modelo de Ising, a imagem mínima corresponde a um toro formado pela caixa central.

Esta técnica reduz os efeitos de borda, mas não os efeitos de tamanho finito.

\section{Longo alcance}

Em sistemas com interação de longo alcance, a energia devido à superfície não se torna desprezível no limite termodinâmico e a eliminação da borda é essencial, pois este efeito não desaparece com o aumento da caixa de simulação, como no caso de sistemas de curto alcance.

A ideia é a mesma, fazer parecer que o sistema a ser simulado faz parte do interior de um sistema enorme com as mesmas propriedades. Uma abordagem utilizada frequentemente é colocar a caixa de simulação em um meio contínuo com propriedades convenientes [33, 53, 54, 55]. Podemos também construir um sistema com muitas camadas de réplicas da caixa de simulação[25, 33, 34]. Além disso, pode-se também colocar a caixa de simulação e suas réplicas 
imersas em um meio contínuo conveniente.

Nenhuma dessas condições de contorno é ideal. Inserir o sistema em um meio contínuo é uma aproximação. Por outro lado, as condições que envolvem réplicas implicam que o líquido está sendo representado por um cristal cuja célula unitária cúbica contém $N$ íons. Ainda existem discussões sobre o melhor método de implementar tais interações [33, 34, 53] e a escolha do método a ser usado deve ser feita em função do modelo físico a ser simulado. Neste trabalho utilizaremos a abordagem de réplicas da caixa de simulação imersas no vácuo.

Na literatura de sistemas de longo alcance utiliza-se quase sempre a convenção de imagem mínima. Por parecer natural, força-se a simetria nos conjuntos de partículas envolvidas na interação com uma determinada partícula. Para cada partícula, define-se uma caixa sob a convenção de imagem mínima (figura 5.1d), que é replicada assim como na figura 5.1a, resultando na configuração da figura 5.1e. Denominaremos este esquema de borda móvel.

A princípio, podemos não impor a convenção de imagem mínima, assim como na figura 5.1b, que é a abordagem que iremos utilizar. Denominaremos este esquema de borda fixa.

Em sistemas de interação de curto alcance, as duas abordagens são absolutamente equivalentes. No entanto, para sistemas de longo alcance, as duas abordagens produzem energias diferentes. Como pode ser visto comparando as representações da mesma configuração nas figuras 5.1b e 5.1e, tanto a caixa central, como as réplicas, mudam ao longo do cálculo da energia. Além disso, para uma mesma configuração, o momento de dipolo total do sistema depende do "ponto de vista" da partícula. 


\section{Energia eletrostática de uma parte}

Vamos considerar inicialmente um sistema $A$, grande e finito. A energia Coulombiana desse sistema é dada por

$$
E_{A}=\frac{1}{2} \sum_{k \in A} \sum_{l \in A}^{\prime} \frac{q_{k} q_{l}}{r_{k l}}
$$

onde $q_{k}$ e $q_{l}$ são as cargas dos íons ou partículas, indexadas por $k$ e por $l$ respectivamente, $r_{k l}=\left|\mathbf{r}_{k l}\right|=\left|\mathbf{r}_{k}-\mathbf{r}_{l}\right|$ é a distância entre eles e a prima sobre o símbolo de somatória indica que o termo $r_{k l}=0$ deve ser omitido da soma. O fator $\frac{1}{2}$ surge do fato da dupla somatória somar a interação de cada par de íons duas vezes.

Queremos olhar para as propriedades termodinâmicas de uma parte do sistema, por exemplo, $\eta_{0}$ da figura 5.2. Para isso, reescreveremos a equação 5.3 de forma que explicite as contribuições de cada parte $\eta$

$$
E_{A}=\frac{1}{2} \sum_{\eta \in A} \sum_{k \in \eta} q_{k} \sum_{\eta^{\prime} \in A} \sum_{l \in \eta^{\prime}}^{\prime} \frac{q_{l}}{r_{k l}}
$$

Essa relação nos permite definir a energia da parte $\eta$ da seguinte forma:

$$
E_{A}=\sum_{\eta \in A} E_{\eta}
$$

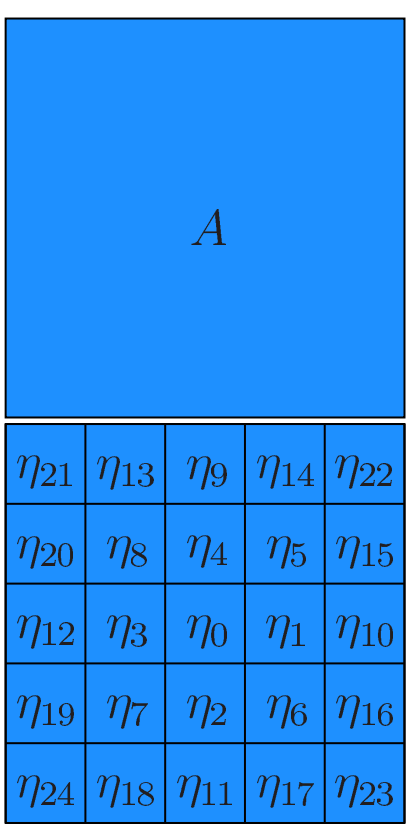

Figura 5.2: Sistema $A$ divido em subsistemas $\eta$ 
onde

$$
E_{\eta}=\frac{1}{2} \sum_{k \in \eta} q_{k} \sum_{\eta^{\prime} \in A} \sum_{l \in \eta^{\prime}}^{\prime} \frac{q_{l}}{r_{k l}}
$$

Simular um sistema grande para obter informação de um sistema pequeno tem um custo computacional muito alto. A ideia é fazer todas as partes $\eta$ do sistema serem idênticas para imitar um sistema grande. Como todas as partes $\eta$ são cópias da caixa de simulação, as partículas contidas na caixa de simulação serão indexadas por $i$ e $j$ e a posição da cópia da partícula $i$ na caixa $\eta$ é $\mathbf{r}_{k}=\mathbf{r}_{i}+\eta$ e a carga $q_{i}=q_{k}$. Nesta nova organização temos que a energia da caixa de simulação, $\eta=0$, é

$$
E_{\eta=0}=\frac{1}{2} \sum_{i} \sum_{\eta} \sum_{j}^{\prime} q_{i} q_{j} \frac{1}{\left|\mathbf{r}_{i j}+\eta\right|}
$$

onde $\eta$ é a posição da réplica $\eta$ e a prima sobre a somatória indica que os termos que $i=j$ e $\eta=0$ devem ser ignorados.

\section{Limite termodinâmico}

No limite termodinâmico, as somatórias da equação 5.7 não comutam,

$$
\sum_{\eta} \sum_{i} \sum_{j} q_{i} q_{j} \frac{1}{\left|\mathbf{r}_{i j}+\eta\right|} \neq \sum_{i} \sum_{j} q_{i} q_{j} \sum_{\eta} \frac{1}{\left|\mathbf{r}_{i j}+\eta\right|}
$$

pois,

$$
\lim _{\left|\eta_{\max }\right| \rightarrow \infty} \sum_{\eta} \frac{1}{\left|\mathbf{r}_{i}-\mathbf{r}_{j}+\eta\right|}=\infty
$$




\subsection{Métodos para o cálculo da energia ele- trostática}

\subsubsection{Soma de Ewald}

A soma de Ewald [43] é uma técnica desenvolvida para calcular a energia Coulombiana de cristais iônicos. O cálculo da energia foi realizado pela primeira vez por Madelung em 1918. Posteriormente, Ewald (1921) encontrou uma série de rápida convergência aplicável ao problema. Logo depois, Born (1921), e posteriormente, Emersleben (1923), modificaram o método para simplificar seu cálculo para redes cristalinas cúbicas [56].

Os livros nos quais esta técnica foi divulgada, por exemplo [33, 34], apresentam o método da seguinte forma: Adiciona-se uma distribuição fictícia de carga com rápido decaimento ao redor de cada íon, o que blinda o potencial coulombiano a longas distâncias. Adiciona-se a mesma distribuição fictícia com carga oposta.

Para o papel da distribuição fictícia, ou nuvem de carga, costuma-se usar a distribuição gaussiana,

$$
\rho_{i}(\mathbf{r})=q_{i}\left(\frac{\alpha}{\pi^{\frac{1}{2}} L}\right)^{3} e^{-\frac{\alpha^{2}\left(\mathbf{r}-\mathbf{r}_{i}\right)^{2}}{L^{2}}}
$$

onde $q_{i}$ é a carga e $\mathbf{r}_{i}$ é a posição da partícula $i$ e $\alpha$ é um parâmetro arbitrário que controla a largura da nuvem de carga.

O método de Ewald assume que o potencial é periódico e o aproxima por uma expansão no espaço recíproco, que é absolutamente adequado a cristais 
iônicos.

A energia total do conjunto das cargas e de suas nuvens e contra-nuvens é dada por [57]

$$
E=E_{1}+E_{2}+E_{3} \quad:
$$

$E_{1}$ é a contribuição para a energia da interação entre cargas $i$ e contra-nuvens $j$ e é efetuada no espaço recíproco,

$$
E_{1}=\frac{1}{2} \sum_{i} \sum_{j} \sum_{\mathbf{n} \neq 0} q_{i} q_{j} \frac{1}{\pi|\mathbf{n}|^{2}} \exp \left(-\frac{\pi^{2}|\mathbf{n}|^{2}}{\alpha^{2}}+i 2 \pi \mathbf{n} \cdot \frac{\mathbf{r}_{i j}}{L}\right) \quad ;
$$

$E_{2}$ representa a energia de interação entre as cargas $i$ e as próprias nuvens e deve ser subtraído do termo anterior e costuma ser denominado autointeração,

$$
E_{2}=-\frac{\alpha}{L \sqrt{\pi}} \sum_{i} q_{i}^{2}
$$

Finalmente, $E_{3}$ representa a energia de interação entre as cargas pontuais e as cargas blindadas, e constitui uma soma no espaço real,

$$
E_{3}=\frac{1}{2} \sum_{i} \sum_{j} q_{i} q_{j}\left(\left(1-\delta_{i j}\right) \frac{1}{r_{i j}} \operatorname{erfc}\left(\alpha r_{i j}\right)+\sum_{\eta \neq \mathbf{0}} \frac{1}{\left|\mathbf{r}_{i j}+\eta\right|} \operatorname{erfc}\left(\alpha \frac{\left|\mathbf{r}_{i j}+\eta\right|}{L}\right)\right)
$$

A função erro complementar $\operatorname{erfc}(x)$ [58] vale um em zero. Implicando que, pelo menos em cristais, no limite de $\alpha \rightarrow 0$ o método de Ewald resgata o calculo explícito do potencial Coulombiano. Além disso, esta função converge rapidamente a zero para valores positivos. Então, somar sobre as camadas de réplicas é cada vez menos relevante. 


\subsubsection{Cálculo direto versus Ewald: a constante de Ma- delung}

Vamos comparar a eficiência do método efetuando o cálculo da constante de Madelung. A constante de Madelung é definida por:

$$
M_{\mathrm{NaCl}}=\sum_{i, j, k=-\infty}^{\infty} \frac{(-1)^{i+j+k}}{\sqrt{i^{2}+j^{2}+k^{2}}}
$$

Representa a energia associada a um único íon no interior de um cristal iônico. A soma sobre as posições $i, j$ e $k$ deve ser executada sobre cubos de largura $L$ cada vez maiores $[59,60,61]$. A afirmação anterior origina-se do resultado do trabalho de Borwein, Borwein e Taylor[60], onde concluíram que a série deve ser somada no mesmo formato da célula unitária. Por curiosidade, a constante de Madelung é dada por [62 ${ }^{1}$

$M_{\mathrm{NaCl}}=-1.7475645946331821906362120355443974034851614366247417581 \ldots$

Na figura 5.3a apresentamos a precisão em casas decimais do cálculo da constante de Madelung efetuados pelo método de Ewald para diferentes fatores $\alpha$ e larguras de sistema, compostos de cubos de lado 2, ou seja 8 íons. A curva com $\alpha=0$ é obtida pelo cálculo direto do potencial Coulombiano. Constata-se que com o aumento de $\alpha$ até um valor entre 0,5 e 0,7 , a taxa de convergência aumenta consideravelmente. Entretanto, acima desse valor o método parece estagnar.

\footnotetext{
${ }^{1}$ http://pi.lacim.uqam.ca/piDATA/madelung.txt
} 


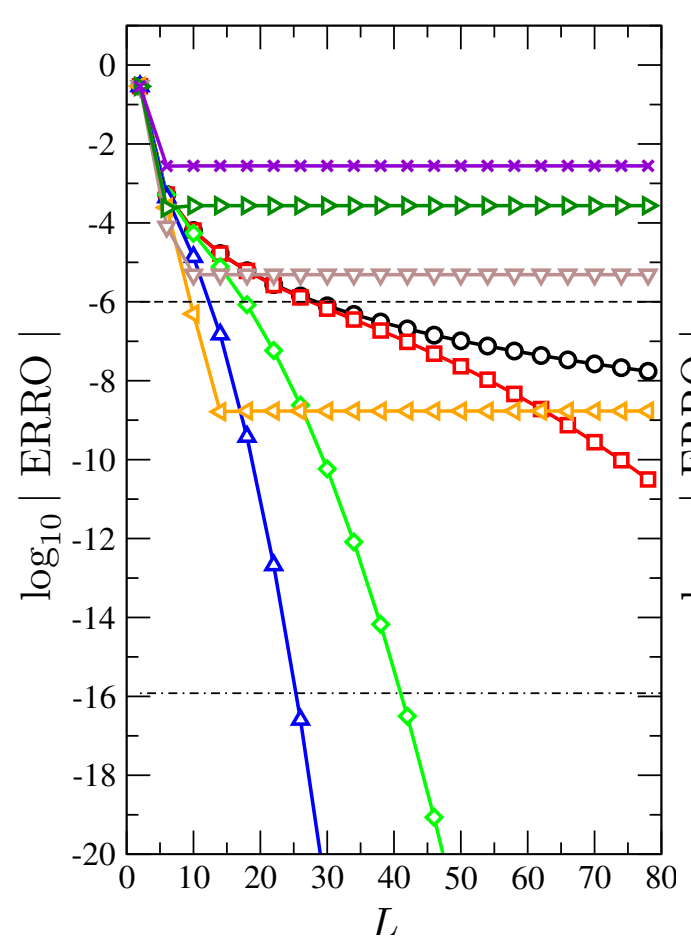

(a) Cúbico

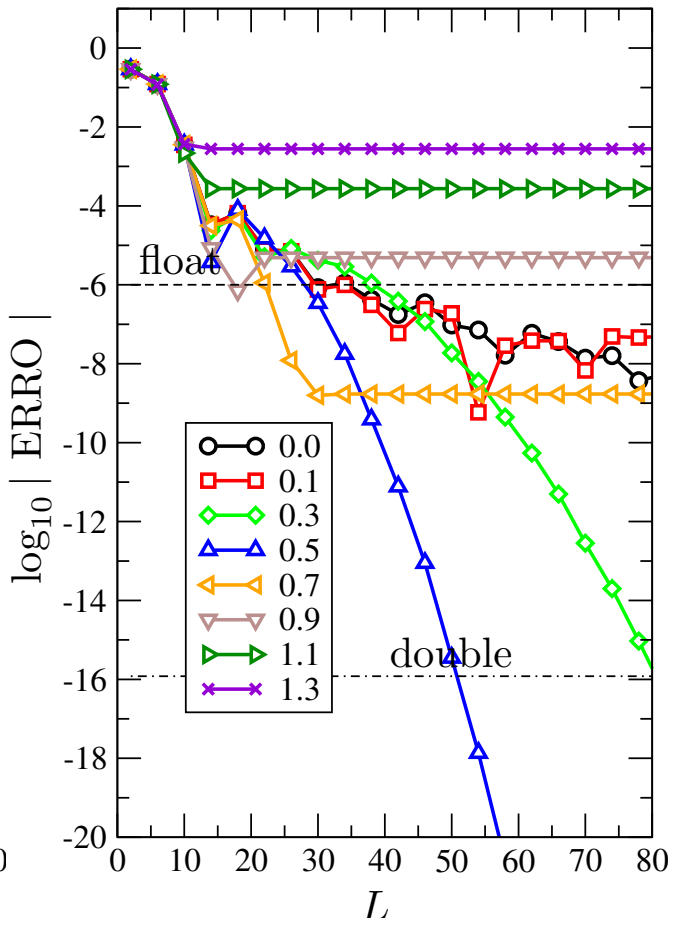

(b) Esférico

Figura 5.3: Precisão em casas decimais do cálculo da constante de Madelung, efetuado pelo método de Ewald sobre réplicas formando cascas cúbicas (a) e sobre réplicas formando cascas esféricas (b), com diferentes parâmetros $\alpha$. Na figura está indicado a quantidade de dígitos significativos dos tipos de ponto flutuante de 32 bits (float) e de 64 bits (double) típicos. Os sistemas são construídos com cubinhos de lado 2: os sistemas cúbicos de lado $L$ são construídos adicionando cubinhos ao redor do cubinho central de maneira tal que se forme um cubo com $L / 2$ cubinhos de largura; os sistemas esféricos de lado $L$ são construídos de maneira tal que a distância entre os centro de todos os cubinhos com o cubinho central, seja menor ou igual a $L-1$.

Por exemplo, vamos supor uma caixa de simulação cúbica de lado 16 e sem réplicas ao redor, então pela figura 5.3a observa-se que o cálculo da energia por partícula do estado cristalino, ordenado como o $\mathrm{NaCl}$, está correto em 5 casas decimais pelo cálculo direto, 6 pelo método de Ewald com $\alpha=0,3$ e $8 \operatorname{com} \alpha=0,5$. Isto reforça a afirmação de alguns autores [33, 34, 63] ao argumentarem que escolhendo o parâmetro $\alpha$ corretamente, a soma na equação 
5.14 (energia do sistema de cargas blindadas) não precisa incluir nenhuma réplica. Na figura 5.3b apresentamos os resultados do mesmo procedimento, porém, realizado sobre cascas esféricas. Vemos neste gráfico outra vantagem do método de Ewald: escolhendo corretamente o valor de $\alpha$, como a série converge mais rápido, ela se assemelha ao comportamento esperado de um sistema de interações de curto alcance, onde o resultado independe da forma da caixa de simulação.

\subsubsection{Cálculo direto versus Ewald para líquidos}

A simulações de líquidos com carga é um assunto muito mais recente do que o estudo analítico de cristais iônicos do início do século passado. Nesta, o líquido é tratado como um cristal para o qual a caixa de simulação representa a célula unitária cuja a configuração varia no tempo. Apesar dessa restrição, tem-se mostrado uma representação válida para a qual podemos resgatar algumas das ferramentas usadas na matéria condensada.

Em 1979, de Leeuw, Perram e Smith [63] publicaram o primeiro de uma série de artigos[49, 64] sobre simulação de sistemas eletrostáticos sob condições periódicas de contorno. Discutem o problema de convergência da série na energia em sistemas homogêneos com interação de longo alcance, testam alguns diferentes fatores de convergência para forçar a convergência absoluta da série em $\eta$. Concluíram que a energia de um sistema neutro em que a soma é feita sobre cascas esféricas é, na notação dessa referência,

$$
H=\sum_{1 \leq i<j \leq N} \frac{1}{L} q_{i} q_{j} \psi\left(\frac{\mathbf{r}_{i j}}{L}\right)+\frac{\xi}{2 L} \sum_{i=1}^{N} q_{i}^{2}+\frac{2 \pi}{3 L^{3}}\left|\sum_{i=1}^{N} q_{i} \mathbf{r}_{i}\right|^{2}
$$


onde $\psi(\mathbf{r})$ é dado por

$$
\psi(\mathbf{r})=\sum_{\mathbf{n}} \frac{\operatorname{erfc}(\alpha|\mathbf{r}+\mathbf{n}|)}{|\mathbf{r}+\mathbf{n}|}+\frac{1}{\pi} \sum_{\mathbf{n} \neq \mathbf{0}}|\mathbf{n}|^{-2} \mathrm{e}^{2 \pi i \mathbf{n} \cdot \mathbf{r}-\pi^{2}|\mathbf{n}|^{2} / \alpha^{2}}
$$

sendo que na notação usada neste trabalho, $\eta=L \mathbf{n}$,

$$
\operatorname{erfc}(x)=1-2 \pi^{\frac{1}{2}} \int_{0}^{x} \mathrm{e}^{t^{2}} d t
$$

é a função erro complementar e

$$
\xi=\sum_{n \neq 0}\left(\frac{\operatorname{erfc}(\alpha|\mathbf{n}|)}{|n|}+\frac{1}{\pi|\mathbf{n}|^{2}} \mathrm{e}^{-\pi^{2}|\mathbf{n}|^{2} / \alpha^{2}}\right)-\frac{2 \alpha}{\pi^{\frac{1}{2}}}
$$

é a parte referente à interação de cada partícula da caixa com as cópias dessa mesma partícula em todas as réplicas das caixas.

Os termos das equações 5.12, 5.13 e 5.14 podem ser reagrupados se separarmos as duas somatórias das equações 5.17 a 5.18 em uma somatória no espaço real, sobre as réplicas $\eta$ da caixa de simulação,

$$
\sum_{\eta} \frac{\operatorname{erfc}\left(\alpha\left|\frac{\mathbf{r}+\eta}{L}\right|\right)}{\left|\frac{\mathbf{r}+\eta}{L}\right|}
$$

e uma soma no espaço recíproco

$$
\frac{1}{\pi} \sum_{\mathbf{k} \neq \mathbf{0}}|\mathbf{k}|^{-2} \exp \left\{2 \pi i \mathbf{k} \cdot \frac{\mathbf{r}}{L}-\pi^{2} \frac{|\mathbf{k}|^{2}}{\alpha^{2} L^{2}}\right\} .
$$

A diferença entre o método de Ewald original e o resultado analítico de de Leeuw, Perram e Smith está no último termo da equação 5.17. Isto significa 
que ao utilizarmos o método de Ewald para calcular a energia eletrostática, equação 5.7, devemos adicionar o último termo da equação 5.17.

Alguns autores de livros de simulação [33, 34] denominam o termo a mais que apareceu na equação 5.17 de correção de dipolo ou termo de dipolo, e atribuem sua existência ao meio em que este conjunto esférico de caixa está imerso.

(Do Allen e Tildesley p.157 [33])

"Os resultados para uma esfera imersa em um bom condutor como um metal $(\varepsilon=\infty)$ e para uma esfera imersa no vácuo são diferentes.

$$
U(\varepsilon=\infty)=U(\varepsilon=1)-\frac{2 \pi}{3 L^{3}}\left|\sum_{i} q_{i} \mathbf{r}_{i}\right|^{2}
$$

Esta equação se aplica no limite de grandes esferas de caixas. No vácuo, a esfera tem uma camada dipolar em sua superfície: o último termo da equação 5.23 cancela isto. ..... O método de Ewald é uma maneira eficiente de calcular $U(\varepsilon=\infty)$. A equação 5.23 permite o uso da soma de Ewald em simulações onde a grande esfera está no vácuo."

No desenvolvimento de de Leeuw, Perram e Smith não há argumentos físicos que resultaram no termo de dipolo. W. Gomes demostrou que este termo resulta da interação entre as cargas pontuais e as nuvens gaussianas na caixa central[57]. O dipolo elétrico total de um cristal iônico é nulo [65], e por isso, é correta a ausência do mesmo no trabalho original de Ewald [43]. 


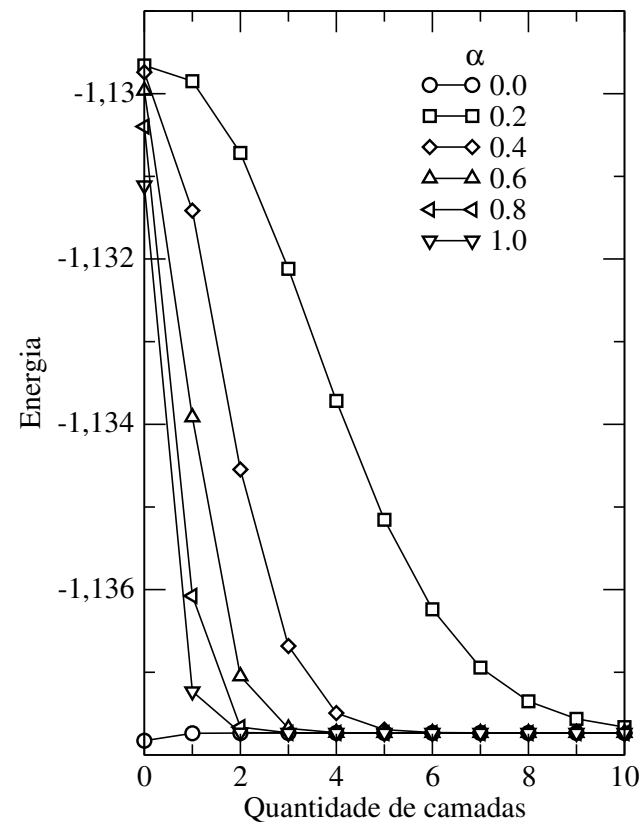

(a) Cristal de lado 2 no centro da caixa

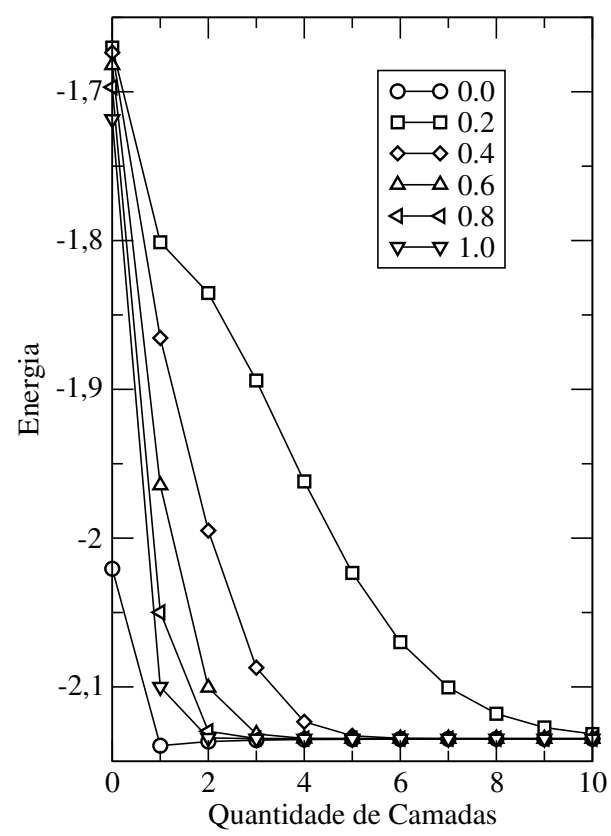

(c) 16 íons aleatórios

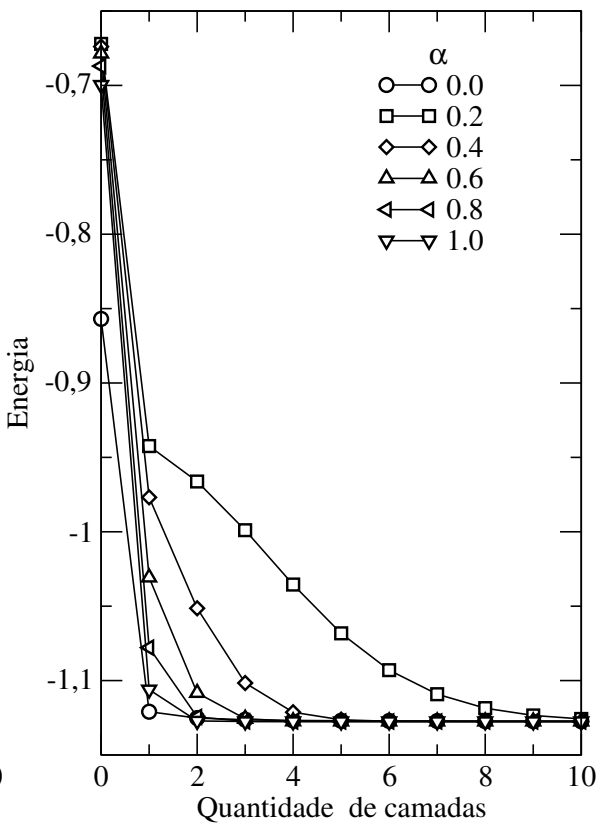

(b) 8 íons aleatórios

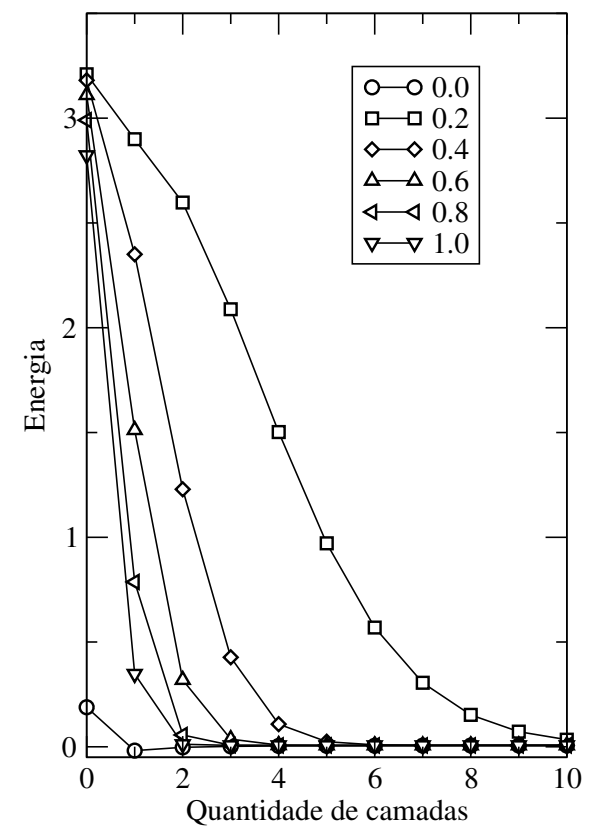

(d) 32 íons aleatórios

Figura 5.4: Convergência do cálculo da energia em função da quantidade de cascas, cúbicas, usando o cálculo direto e o método de Ewald com o termo de dipolo para algumas configurações: um cristal com 8 íons(a) e 3 configurações aleatórios com 8 íons (b), 16 íons (c) e 32 íons (d). As curvas representam diferentes valores para o parâmetro $\alpha . \alpha=0$ corresponde ao cálculo direto do potencial coulombiano. Nestes casos, nos cálculos envolvendo poucas réplicas, o cálculo direto apresentou resultados melhores que o método de Ewald para os vários parâmetros $\alpha$. 
Na figura 5.4, apresentamos o cálculo da energia do sistemas para algumas configurações arbitrárias, diferentes da configuração cristalina utilizada para o cálculo da constante de Madelung. A soma foi realizada sobre cascas cúbicas. Observa-se que o valor obtido pelo cálculo direto, $\alpha=0$, para nenhuma réplica, apresenta resultado mais próximo do resultados para muitas réplicas do que os resultados obtidos pelo método de Ewald.

\subsubsection{Importância da correção de dipolo}

Para demonstrar a influência do termo de dipolo sobre os resultados de uma simulação, são apresentados as distribuições de energias obtidas em simulações que usaram o método direto, o método de Ewald e o método de Ewald com o termo de dipolo, fig. 5.5a. Neste gráfico há uma notável diferença entre o método de Ewald em relação aos outros métodos. A diferença entre o método de Ewald com o termo de dipolo e o calculo direto é imperceptível.

De Leeuw e Perram, através de dinâmica molecular, reportaram [48] que propriedades termodinâmicas e algumas propriedades dinâmicas são independentes da natureza dielétrica do meio que envolve o sistema. Em outras palavras, eles afirmaram que a inclusão do termo de dipolo resulta em funções distribuição radial similares, onde a diferença era menor que o erro estatístico. Na figura 5.5b, é apresentada a distribuição dos valores do termo de dipolo. A grande diferença entre o método de Ewald com e sem termo de dipolo prova que os estados microscópicos de equilíbrio gerados são muito influenciados pelo termo de dipolo. 


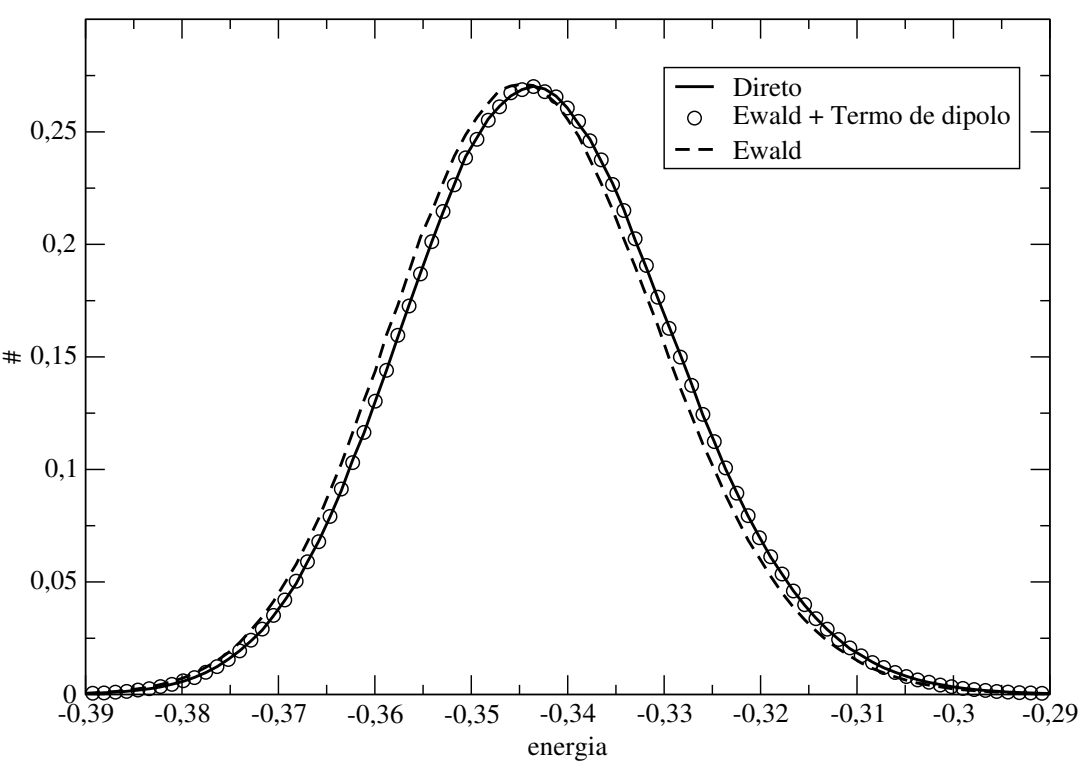

(a) Distribuição de energia

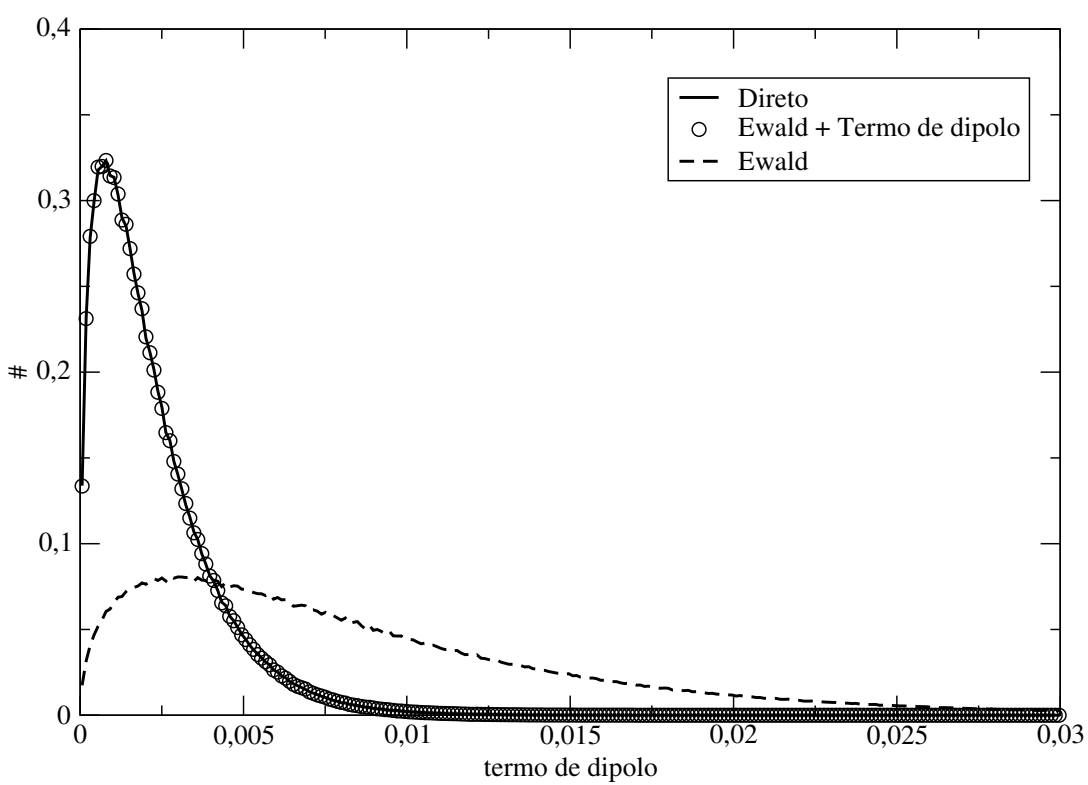

(b) Distribuição da componente da energia devido ao dipolo

Figura 5.5: Histogramas da distribuição de energia(a) e do termo de dipolo(b) do modelo LRPM ao se computar a energia com o cálculo direto, método de Ewald com e sem o termo de dipolo. Cada histograma é resultado de 8 simulações de 1 milhão de passos de Monte Carlo, com 512 partículas, temperatura $t=1.0$ e a caixa de simulação tem dimensão 16x16x16. As simulações foram alimentadas com 3 conjunto idênticos de 8 sementes diferentes. 
Contrariando de Leeuw e Perram, obtivemos diferença maior que o erro estatístico na distribuição de energia e configurações de equilíbrio com dipolos consideravelmente diferentes. O contraste da nossa conclusão em relação a distribuição de energia, propriedades termodinâmicas, pode ser justificado pois o erro estatístico aceitável, considerando a escassez de recursos computacionais há 30 anos atrás, era maior. Além disso, dinâmica molecular costuma demorar mais para alcançar estados de equilíbrio.

\subsection{Cálculo eficiente da diferença de energia: a função $f\left(\mathbf{r}_{i j}\right)$}

Em simulações, é conveniente reduzir ao máximo o custo computacional, proporcional a quantidade de operações, do cálculo da diferença de energia entre estados. Em sistemas com interações de longo alcance e condições "periódicas" de contorno isto requer mais cuidado.

\subsubsection{Função $f\left(\mathbf{r}_{i j}\right)$}

Vamos separar toda a parte espacial do potencial Coulombiano combinado de todas as réplicas e a escolha da condição de contorno, em uma função, a função $f\left(\mathbf{r}_{i}-\mathbf{r}_{j}\right)$.

No limite termodinâmico, as somatórias da equação 5.7 não comutam,

$$
\sum_{\eta} \sum_{i} \sum_{j} q_{i} q_{j} \frac{1}{\left|\mathbf{r}_{i j}+\eta\right|} \neq \sum_{i} \sum_{j} q_{i} q_{j} \sum_{\eta} \frac{1}{\left|\mathbf{r}_{i j}+\eta\right|}
$$


pois,

$$
\lim _{\left|\eta_{\max }\right| \rightarrow \infty} \sum_{\eta} \frac{1}{\left|\mathbf{r}_{i}-\mathbf{r}_{j}+\eta\right|}=\infty
$$

Para poder manipular as somatórias, vamos considerar que o sistema, grande ou não, é finito. Assim, as somatórias da eq. 5.7 comutam.

Isto é bastante claro no cálculo direto. Na abordagem do método de Ewald, o sistema é infinito. Por outro lado, quando há rápida convergência da série é permitido rearranjar as somatórias. Com isso, a componente espacial da energia pode ser calculada independentemente.

A função $f\left(\mathbf{r}_{i}-\mathbf{r}_{j}\right)$, é definida a menos de uma constante, devido a neutralidade, por:

$$
E=\frac{1}{2} \sum_{i} q_{i} \sum_{j} q_{j} f\left(\mathbf{r}_{i}-\mathbf{r}_{j}\right)
$$

Podemos comparar, a menos de uma constante, as funções $f\left(\mathbf{r}_{i j}\right)$ oriundas de diferentes métodos ponto a ponto, dessa forma, independente de configuração.

Vamos mostrar como se gera esta função em alguns casos.

\section{Soma direta na forma $f\left(\mathbf{r}_{i j}\right)$}

É conveniente reorganizar a soma direta, equação 5.7, dada por

$$
E=\sum_{i} q_{i}\left(\sum_{j \neq i} \frac{1}{2} \frac{q_{j}}{\left|\mathbf{r}_{i}-\mathbf{r}_{j}\right|}+\sum_{j} \sum_{\eta \neq 0} \frac{1}{2} \frac{q_{j}}{\left|\eta+\mathbf{r}_{i}-\mathbf{r}_{j}\right|}\right)
$$


na forma da equação 5.26, então

$$
f\left(\mathbf{r}_{i}-\mathbf{r}_{j}\right)=\frac{1-\delta_{i, j}}{\left|\mathbf{r}_{i}-\mathbf{r}_{j}\right|}+\sum_{\eta \neq 0} \frac{1}{\left|\eta+\mathbf{r}_{i}-\mathbf{r}_{j}\right|}
$$

Quando $i=j$, o termo $\frac{1-\delta_{i, j}}{\left|\mathbf{r}_{i}-\mathbf{r}_{j}\right|}$ fica indeterminado, neste caso, este termo deve ser interpretado como nulo.

\section{Soma de Ewald na forma $f\left(\mathbf{r}_{i j}\right)$}

Como antes, vamos reescrever estas expressões para ficar na forma da equação 5.26. Ou seja,

$$
f_{\text {Ewald }}\left(\mathbf{r}_{i j}\right)=f_{1}\left(\mathbf{r}_{i j}\right)+f_{2}\left(\mathbf{r}_{i j}\right)+f_{3}\left(\mathbf{r}_{i j}\right),
$$

onde

$$
\begin{gathered}
f_{1}\left(\mathbf{r}_{i j}\right)=\sum_{\mathbf{n} \neq 0} \frac{1}{\pi|\mathbf{n}|^{2}} \exp \left(-\frac{\pi^{2}|\mathbf{n}|^{2}}{\alpha^{2}}+i 2 \pi \mathbf{n} \cdot \frac{\mathbf{r}_{i j}}{L}\right), \\
f_{2}\left(\mathbf{r}_{i j}\right)=-2 \frac{\alpha}{L \sqrt{\pi}} \delta i j
\end{gathered}
$$

e

$$
f_{3}\left(\mathbf{r}_{i j}\right)=\left(1-\delta_{i j}\right) \frac{1}{r_{i j}} \operatorname{erfc}\left(\alpha \frac{r_{i j}}{L}\right)+\sum_{\eta \neq \mathbf{0}} \frac{1}{\left|\mathbf{r}_{i j}+\eta\right|} \operatorname{erfc}\left(\alpha \frac{\left|\mathbf{r}_{i j}+\eta\right|}{L}\right)
$$

\section{Correção de Dipolo na forma $f\left(\mathbf{r}_{i j}\right)$}

É preciso reescrever a expressão da correção de dipolo (eq. 5.23) [33]. 


$$
\begin{aligned}
\left|\sum_{i} q_{i} \mathbf{r}_{i}\right|^{2} & =\sum_{i} q_{i} \mathbf{r}_{i} \cdot \sum_{j} q_{j} \mathbf{r}_{j} \\
& =\sum_{i} \sum_{j} q_{i} q_{j} \mathbf{r}_{i} \cdot \mathbf{r}_{j} \\
& =\frac{1}{2} \sum_{i} \sum_{j} q_{i} q_{j}\left(2 \mathbf{r}_{i} \cdot \mathbf{r}_{j}\right) \\
& =\frac{1}{2} \sum_{i} \sum_{j} q_{i} q_{j}(\underbrace{2 \mathbf{r}_{i} \cdot \mathbf{r}_{j}-\mathbf{r}_{i} \cdot \mathbf{r}_{i}-\mathbf{r}_{j} \cdot \mathbf{r}_{j}}_{-\left(\mathbf{r}_{i}-\mathbf{r}_{j}\right)^{2}}+\mathbf{r}_{i} \cdot \mathbf{r}_{i}+\mathbf{r}_{j} \cdot \mathbf{r}_{j})
\end{aligned}
$$

e os termos $\sum_{i} q_{i} \sum_{j} q_{j} r_{j}^{2}$ se anulam, pois por construção, $\sum_{i} q_{i}=0$. Dessa forma temos que

$$
\left|\sum_{i} q_{i} \mathbf{r}_{i}\right|^{2}=\frac{1}{2} \sum_{i} \sum_{j} q_{i} q_{j}\left(-r_{i j}^{2}\right)
$$

Portanto, o termo de correção na energia que usamos em nossas simulações terá a forma

$$
U_{D}=\frac{1}{2} \sum_{i} q_{i} \sum_{j} q_{j}\left(-\frac{2 \pi}{3 L^{3}} r_{i j}^{2}\right)
$$

onde $r_{i j}^{2}=\left(\mathbf{r}_{i}-\mathbf{r}_{j}\right) \cdot\left(\mathbf{r}_{i}-\mathbf{r}_{j}\right)$, ficando evidente que esta grandeza independe do referencial.

\subsubsection{Cálculo da diferença de energia}

Na dinâmica de Metropolis, as configurações são geradas em função do cálculo da diferença de energia. A varredura no espaço de configurações é realizada 
comparando a energia entre dois estados. Em sistemas carregados, é comum, para se obter a diferença de energia entre os dois estados, calcular a energia dos dois estados separadamente. Em simulações de modelos simplificados, de curto alcance, como o modelo de Ising, é tradição[66] obter analiticamente uma expressão da diferença de energia para cada processo.

O cálculo da energia em sistemas carregados envolve todas as partículas do sistema e por isso o custo computacional é muito maior. Aplicar esta técnica tradicional é muito vantajoso computacionalmente, tanto em relação ao tempo como em relação ao acumulo de erro numérico, ambos por reduzir a quantidade de operações. Por outro lado, é trabalhoso obter analiticamente expressões para cada processo da dinâmica. Como exemplo, vamos calcular da diferença de energia ao se mover uma partícula carregada (sec. 5.5.2), ao se adicionar um par neutro de partículas carregas (sec. 5.5.2), e ao se trocar a carga de duas partículas (sec. 5.5.2).

A energia escrita na forma

$$
U=\frac{1}{2} \sum_{i} \sum_{j} q_{i} q_{j} f\left(\mathbf{r}_{i}-\mathbf{r}_{j}\right)
$$

onde $r_{i}$ é a posição do sítio $i$, esconde na função $f\left(\mathbf{r}_{i}-\mathbf{r}_{j}\right)$ os detalhes do método e da condição de contorno escolhidos. Assim, os resultados seguintes independem dessa escolha.

\section{Movendo uma carga}

Deslocar o sítio $\alpha$, ou seja, $r_{\alpha} \rightarrow r_{\alpha}^{\prime}$ implica que o conjunto de posições $r_{i}$ vai ter o elemento $\alpha$ alterado e o novo conjunto será representado por $r_{i}^{\prime}$. 
A energia do sistema com o sítio $\alpha$ em uma nova posição é dada por

$$
U^{\prime}=\frac{1}{2} \sum_{i} \sum_{j} q_{i} q_{j} f\left(\mathbf{r}_{i}^{\prime}-\mathbf{r}_{j}^{\prime}\right)
$$

onde $\mathbf{r}_{j}^{\prime}$ é a posição do sítio $j$ na nova configuração. Ou seja, a diferença de energia devido ao deslocamento do sítio $\alpha$ é

$$
\delta U=U^{\prime}-U=\frac{1}{2} \sum_{i} \sum_{j} q_{i} q_{j}\left(f\left(\mathbf{r}_{i}^{\prime}-\mathbf{r}_{j}^{\prime}\right)-f\left(\mathbf{r}_{i}-\mathbf{r}_{j}\right)\right)
$$

Reescrevendo de forma que explicite as diferenças entre os potenciais das partículas que não se movem, temos para a soma em $j$

$$
\delta U=\frac{1}{2} \sum_{i}\left(\sum_{j \neq \alpha} q_{i} q_{j}\left(f\left(\mathbf{r}_{i}^{\prime}-\mathbf{r}_{j}\right)-f\left(\mathbf{r}_{i}-\mathbf{r}_{j}\right)\right)+q_{i} q_{\alpha}\left(f\left(\mathbf{r}_{i}^{\prime}-\mathbf{r}_{\alpha}^{\prime}\right)-f\left(\mathbf{r}_{i}-\mathbf{r}_{\alpha}\right)\right)\right),
$$

onde $\mathbf{r}_{j}=\mathbf{r}_{j}^{\prime}$ para $j \neq \alpha$ e para a soma em $i$

$$
\begin{aligned}
\delta U & =\frac{1}{2} \sum_{i \neq \alpha}(\sum_{j \neq \alpha} q_{i} q_{j} \underbrace{\left(f\left(\mathbf{r}_{i}-\mathbf{r}_{j}\right)-f\left(\mathbf{r}_{i}-\mathbf{r}_{j}\right)\right)}_{0}+q_{i} q_{\alpha}\left(f\left(\mathbf{r}_{i}-\mathbf{r}_{\alpha}^{\prime}\right)-f\left(\mathbf{r}_{i}-\mathbf{r}_{\alpha}\right)\right)) \\
& +\frac{1}{2} \sum_{j \neq \alpha} q_{\alpha} q_{j}\left(f\left(\mathbf{r}_{\alpha}^{\prime}-\mathbf{r}_{j}\right)-f\left(\mathbf{r}_{\alpha}-\mathbf{r}_{j}\right)\right) \\
& +\frac{1}{2} q_{\alpha} q_{\alpha}\left(f\left(\mathbf{r}_{\alpha}^{\prime}-\mathbf{r}_{\alpha}^{\prime}\right)-f\left(\mathbf{r}_{\alpha}-\mathbf{r}_{\alpha}\right)\right),
\end{aligned}
$$


que implica que, se $f(a-b)=f(b-a)$,

$\delta U=\sum_{i \neq \alpha} q_{i} q_{\alpha}\left(f\left(\mathbf{r}_{i}-\mathbf{r}_{\alpha}^{\prime}\right)-f\left(\mathbf{r}_{i}-\mathbf{r}_{\alpha}\right)\right)+\frac{1}{2} q_{\alpha} q_{\alpha}\left(f\left(\mathbf{r}_{\alpha}^{\prime}-\mathbf{r}_{\alpha}^{\prime}\right)-f\left(\mathbf{r}_{\alpha}-\mathbf{r}_{\alpha}\right)\right)$

a expressão final é dada por

$$
\delta U=\sum_{i \neq \alpha} q_{i} q_{\alpha}\left(f\left(\mathbf{r}_{i}-\mathbf{r}_{\alpha}^{\prime}\right)-f\left(\mathbf{r}_{i}-\mathbf{r}_{\alpha}\right)\right)
$$

\section{Inserindo um par neutro de cargas}

Vamos calcular a diferença de energia ao se adicionar um par neutro de partículas, denominadas $\alpha$ e $\beta$, para energia escrita na forma

$$
U=\frac{1}{2} \sum_{i \neq\{\alpha, \beta\}} \sum_{j \neq\{\alpha, \beta\}} q_{i} q_{j} f\left(\mathbf{r}_{i}-\mathbf{r}_{j}\right)
$$

onde $\mathbf{r}_{i}$ é a posição do sítio $i$.

A energia do sistema com as partículas $\alpha$ e $\beta$ é dada por

$$
\begin{aligned}
2 U= & \sum_{i \neq\{\alpha, \beta\}} q_{i}\left(q_{\alpha} f\left(\mathbf{r}_{i}-\mathbf{r}_{\alpha}\right)+q_{\beta} f\left(\mathbf{r}_{i}-\mathbf{r}_{\beta}\right)+\sum_{j \neq\{\alpha, \beta\}} q_{j} f\left(\mathbf{r}_{i}-\mathbf{r}_{j}\right)\right) \\
& +q_{\alpha} q_{\alpha} f\left(\mathbf{r}_{\alpha}-\mathbf{r}_{\alpha}\right)+q_{\alpha} q_{\beta} f\left(\mathbf{r}_{\alpha}-\mathbf{r}_{\beta}\right)+\sum_{j \neq\{\alpha, \beta\}} q_{\alpha} q_{j} f\left(\mathbf{r}_{\alpha}-\mathbf{r}_{j}\right) \\
& +q_{\beta} q_{\alpha} f\left(\mathbf{r}_{\beta}-\mathbf{r}_{\alpha}\right)+q_{\beta} q_{\beta} f\left(\mathbf{r}_{\beta}-r_{\beta}\right)+\sum_{j \neq\{\alpha, \beta\}} q_{\beta} q_{j} f\left(\mathbf{r}_{\beta}-\mathbf{r}_{j}\right),
\end{aligned}
$$

Devido a neutralidade do par adicionado, $q_{\alpha} q_{\alpha}=q_{\beta} q_{\beta}=1$ e $q_{\alpha} q_{\beta}=-1$, 
temos:

$$
\begin{aligned}
2 \Delta U & =\sum_{i \neq\{\alpha, \beta\}} q_{i}\left(+q_{\alpha} f\left(\mathbf{r}_{i}-\mathbf{r}_{\alpha}\right)+q_{\beta} f\left(\mathbf{r}_{i}-\mathbf{r}_{\beta}\right)\right) \\
& +f\left(\mathbf{r}_{\alpha}-\mathbf{r}_{\alpha}\right)-f\left(\mathbf{r}_{\alpha}-\mathbf{r}_{\beta}\right)+\sum_{j \neq\{\alpha, \beta\}} q_{\alpha} q_{j} f\left(\mathbf{r}_{\alpha}-\mathbf{r}_{j}\right) \\
& +f\left(\mathbf{r}_{\beta}-\mathbf{r}_{\beta}\right)-f\left(\mathbf{r}_{\beta}-\mathbf{r}_{\alpha}\right)+\sum_{j \neq\{\alpha, \beta\}} q_{\beta} q_{j} f\left(\mathbf{r}_{\beta}-\mathbf{r}_{j}\right) .
\end{aligned}
$$

Devido a simetria, se resume finalmente em:

$$
\Delta U=f(\mathbf{0})-f\left(\mathbf{r}_{\alpha}-\mathbf{r}_{\beta}\right)+\sum_{i \neq\{\alpha, \beta\}} q_{i}\left(+q_{\alpha} f\left(\mathbf{r}_{i}-\mathbf{r}_{\alpha}\right)+q_{\beta} f\left(\mathbf{r}_{i}-\mathbf{r}_{\beta}\right)\right) .
$$

\section{Trocando}

Trocar a carga de duas partículas equivale a retirar o par de partículas e inserir um par de sinal oposto na mesma posição. Resultando em :

$$
\Delta U=-2 q_{\alpha} \sum_{i \neq \alpha} q_{i}\left(+f\left(\mathbf{r}_{i}-\mathbf{r}_{\alpha}\right)-f\left(\mathbf{r}_{i}-\mathbf{r}_{\beta}\right)\right)
$$

\subsection{Comentários sobre custo computacional e precisão numérica}

\section{Sobre precisão numérica.}

O balanço entre o custo computacional e a precisão numérica em simulações com carga é um assunto recorrente[44]. Em simulação de líquidos: não há a constante de Madelung para ser usada como referência; não é ideal usar o 
método de Ewald como referência, pois líquidos não possuem simetria translacional; e não se deve também usar o cálculo direto sem a inclusão de réplicas, pois o efeito de borda são relevantes.

Tendo isto em mente, realizar o cálculo da energia com muitas casas de precisão não significa necessariamente um resultado melhor, pois mais réplicas significa um sistema mais "periódico". Para os objetivos dessa tese, o cálculo direto é mais apropriado porque é simples, estável, aplicável em duas dimensões e suficientemente preciso.

\section{Sobre custo computacional}

Originalmente, tanto o método de Ewald como o cálculo direto têm custo computacional que escala com $(n N)^{2}$, onde $N$ é a quantidade de partículas da caixa de simulação e $n$ é a quantidade de réplicas da caixa de simulação mais um.

Existem tentativas de deixar o método de Ewald mais eficiente. A mais famosa, por ser a única exata, é o método de Ewald otimizado por Perram, Petersen e de Leuuw, tendo custo que escala com $N^{\frac{3}{2}}$ [52]. Outras tentativas são aproximações.

Provavelmente, o método de cálculo mais usado em grandes clusters atualmente é devido a Greengard e Rokhlin, denominado fast multipole method (FFM)[42], que aproxima a influência de conjuntos de cargas distantes por multipolos. O FFM tem uma estrutura de árvore, que é dito reduzir o custo computacional, passando a escalar com $N \ln N$. Aperfeiçoaram o método e o custo passou a escalar com $N$ [52]. Entretanto, por causa da complexidade das funções matemáticas envolvidas, o método só é vantajoso para sistemas 
grandes.

A introdução da função $f\left(\mathbf{r}_{i j}\right)$ associada com as equações $5.42,5.46$ e 5.47, permite alcançar um custo computacional que escala com a quantidade de partículas da mesma forma que o custo computacional do fast multipole method otimizado. Se usado no cálculo direto da energia eletrostática, devido a simplicidade das operações envolvidas, o cálculo da diferença de energia é notoriamente mais rápido e sem aproximações.

Para simulações em reticulados, é conveniente gerar previamente uma tabela com todos os possíveis vetores de distância entre cargas, com o método e as condições de contorno escolhidos. Vamos considerar, por conveniência, apenas o caso onde as réplicas das caixa são dispostas ao redor da caixa de simulação de maneira simétrica. Assim, para cada caixa nas coordenadas $\left\{\eta_{x}, \eta_{y}, \eta_{z}\right\}$ existe uma outra caixa nas coordenadas $\left\{-\eta_{x},-\eta_{y},-\eta_{z}\right\}$. Então podemos escrever

$$
\sum_{\eta}|\boldsymbol{\eta}-\mathbf{r}|=\sum_{\eta}|\boldsymbol{\eta}+\mathbf{r}|
$$

para podemos definir $f\left(\mathbf{r}_{i}-\mathbf{r}_{j}\right)=f\left(\left\{\left|\mathbf{x}_{i}-\mathbf{x}_{j}\right|,\left|\mathbf{y}_{i}-\mathbf{y}_{j}\right|,\left|\mathbf{z}_{i}-\mathbf{z}_{j}\right|\right\}\right)$.

Criamos uma tabela para cada um dos possíveis vetores distância entre as cargas, incluindo as condições de contorno. Para reticulados pequenos, é possível que a tabela inteira seja alocada na memória cache do processador, aumentando em muito a performance. Para sistemas no contínuo, é possível interpolar os pontos dessa tabela. Esta função talvez possa ser construída usando outros métodos, tais como a expansão em multipolos e reaction field. 


\section{Capítulo 6}

\section{Modelo para transição}

\section{gel-fluido em membranas}

\section{carregadas em solução aquosa}

Neste capítulo será unido o modelo de membrana do capítulo 3 com o modelo de solução iônica do capítulo 5, no intuito de descrever a transição ordem - desordem de membranas carregadas em solução. Na seção 1 descrevemos dois modelos que levam em conta as cargas da cabeça polar, ionizável ou não. Na seção 2 apresentamos testes do modelo para a solução iônica na presença de um plano carregado e na seção 3 apresentamos resultados exploratórios para os dois modelos. 


\subsection{Construção do modelo}

As membranas ditas carregadas são formadas por lipídios cuja cabeça polar se dissocia em solução, e as membranas ditas neutras são formadas por lipídios que possuem um dipolo na cabeça polar. Os modelos de Doniach et alii e o nosso modelo de membrana neutra não consideram explicitamente a carga das cabeças polares lipídicas, e a interação dipolar entre lipídios neutros foi incluída de maneira efetiva nas interações entre primeiros vizinhos. A distribuição de carga das cabeças são bem representadas por um dipolo como na figura $1.9 \mathrm{~b}$.

Para incluir dissociação, é preciso que o modelo tenha representações distintas do lipídio associado e do lipídio dissociado. Vamos passar a representar a cabeça polar lipídica por um par neutro de partículas carregadas, como na figura 1.9c. Definimos dois novos modelos: o modelo de membrana neutra, formada por lipídios que não ionizam, ou seja, a carga positiva sempre está ligada à parte negativa e os dipolos têm sentidos paralelos; e o modelo de membrana carregada, ou carregável, formada por lipídios nos quais a parte positiva da cabeça polar é livre. O modelo primitivo restrito na rede (LRPM) suporta esta representação para o sistema membrana mais solução. Então escolhemos descrever toda à parte carregada pelo LRPM e descrever toda a parte referente à ordem-desordem das cadeias lipídicas pelo nosso modelo neutro de membrana, o DLG. Os dois modelos são definidos em redes distintas, a rede de cargas e a rede de cadeias.

Definimos a rede de cargas em um reticulado tridimensional paralelepídico, de base quadrada de área $L \times L$ e altura $L_{z}$. A caixa de simulação das cargas é 


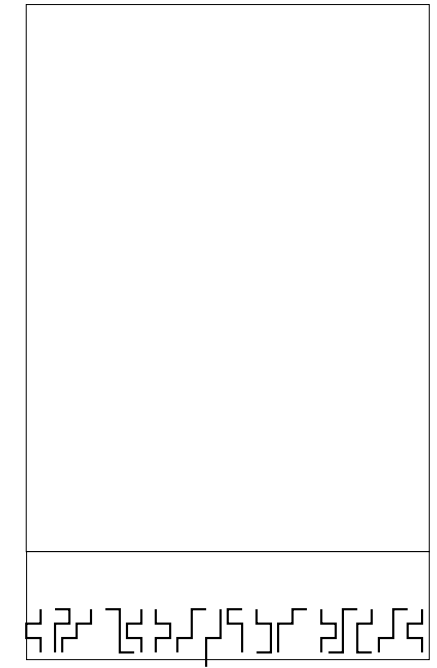

(a) rede de cadeias

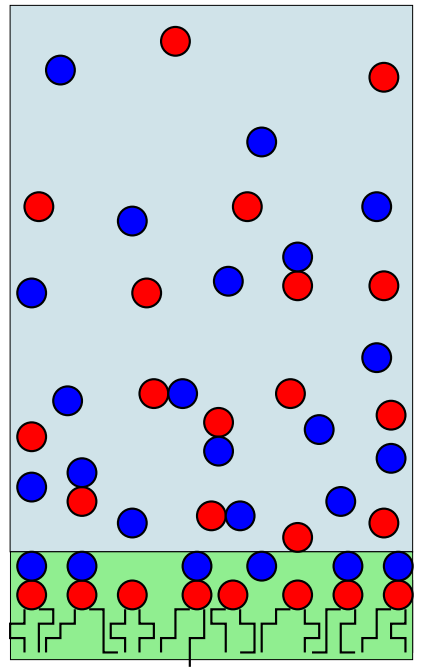

(b) rede composta

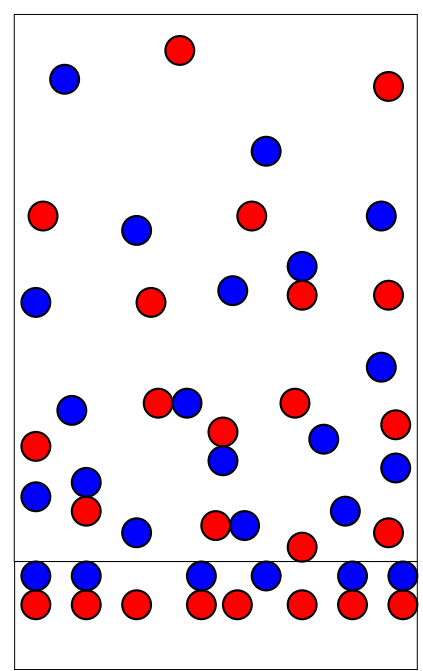

(c) rede de cargas

Figura 6.1: Diferentes representações de lipídio.

dividida em $L_{z}$ camadas paralelas a base. Utilizamos 20 camadas de réplicas ao redor dessa caixa de simulação nos eixos do plano e a caixa é fechada na primeira e última camada. A energia da configuração das cargas é dada por

$$
E_{\text {carga }}=\lambda \sum_{k} \sum_{l} \frac{q_{k} q_{l}}{\left|r_{k l}\right|}
$$

onde $\lambda$ é um parâmetro arbitrário que pode ser ajustado ou estimado e as somatórias são realizadas sobre todas as partículas, indexadas por $k$ e $l, r_{k l}$ é a distância entre as partículas, com cargas que assumem os valores $q= \pm 1$.

A parte ordem-desordem das cadeias de carbono dos lipídios formadores da membrana é descrita na rede de cadeias, e é definida em um reticulado bidimensional quadrado de lado $L$. O sítio na posição $(x, y)$ na rede de cadeias está associado univocamente ao sítio na mesma posição na primeira camada da rede de cargas, $(x, y, z=0)$. Para cada sítio ocupado por um lipídio na 
rede de cadeias, $\sigma^{2}=1$, existe uma carga negativa na mesma posição na rede de cargas. A energia da configuração na rede de cadeias é dada por

$$
E_{\text {cadeias }}=-J \sum_{(i j)} \sigma_{i} \sigma_{j}-\Delta \sum_{(i j)} \sigma_{i} \sigma_{j}\left(\sigma_{i}+\sigma_{j}\right)-K \sum_{(i j)} \sigma_{i}^{2} \sigma_{j}^{2}
$$

onde a soma em $i$ é realizada sobre toda a rede, e $j$ indexa metade dos quatro primeiros vizinhos do sítio $i^{1}$. O sítio assume os estados: ordenado, $\sigma=1$; desordenado, $\sigma=-1$; e vazio, $\sigma=0$, quando não há lipídio.

Os lipídios estão restritos ao plano da caixa de simulação das cadeias e, portanto, as cargas negativas correspondentes estão restritas à primeira camada da caixa de cargas, $z=0$. Os íons da solução, os quais não possuem correspondência com os lipídios da membrana, estão restritos ao espaço restante, ou seja, toda a rede com exceção da primeira camada, $z \neq 0$.

Então, a única diferença entre os modelos, com e sem dissociação, que denominaremos DLG carregado e DLG dipolar, é que no modelo com dissociação, a carga positiva se move livremente, e, no modelo sem dissociação, a carga positiva imediatamente acima $(x, y, 1)$ da carga negativa $(x, y, 0)$ está associada a uma cadeia e se move junto com ela.

\subsection{Teste do modelo: plano carregado e solução iônica}

Estamos acostumados a trabalhar com modelos onde o interesse se restringe às propriedade de bulk, e os efeitos de borda devem ser minimizados. Para

\footnotetext{
${ }^{1}$ Para que a energia de interação entre pare não seja computada em duplicidade
} 
este fim, existem as condições periódicas de contorno. Neste modelo, a distribuição de cargas próxima à superfície é uma das propriedades de interesse. Em outras palavras, estamos interessados nos efeitos de uma única borda, a borda devida à membrana. As outras bordas devem se construídas com condições periódicas de contorno. Entretanto, não é possível impor condições periódicas de contorno à borda oposta a borda da membrana, pois não há fluxo de partículas na superfície do plano. Então, por consistência, a caixa de simulação é fechada na face da membrana $(z=0)$ e na face oposta $(z=L z)$.

Antes de juntar o modelo de membrana com o modelo de solução iônica, vamos efetuar alguns testes do sistema carregado, considerando uma placa carregada de cargas fixas e a solução iônica.

Na figura 6.2 apresentamos um estudo do efeito do tamanho de $L_{z}$ sobre a distribuição de partículas ao longo do eixo z. O sistema considerado é uma placa totalmente carregada e outra com densidade de carga de $20 \%$ na qual as cargas foram distribuídas em posições aleatórias fixas. Nas solução estão presentes os contraíons e os íons do sal adicionado $(\rho=0, \rho=0,0009766$ e $\rho=0,0078125)$. Verifica-se, em todos os casos, um decaimento aparentemente exponencial para um patamar.

As figuras ilustram dois efeitos importantes do tamanho finito. Para uma dada concentração de sal, espera-se um patamar referente a esta concentração. No entanto, observamos que o valor do patamar é função do comprimento da caixa de simulação, e se aproxima do valor esperado com o aumento do comprimento da caixa. Além disso, a convergência para o valor da concentração da solução piora à medida que a concentração de sal aumenta. 


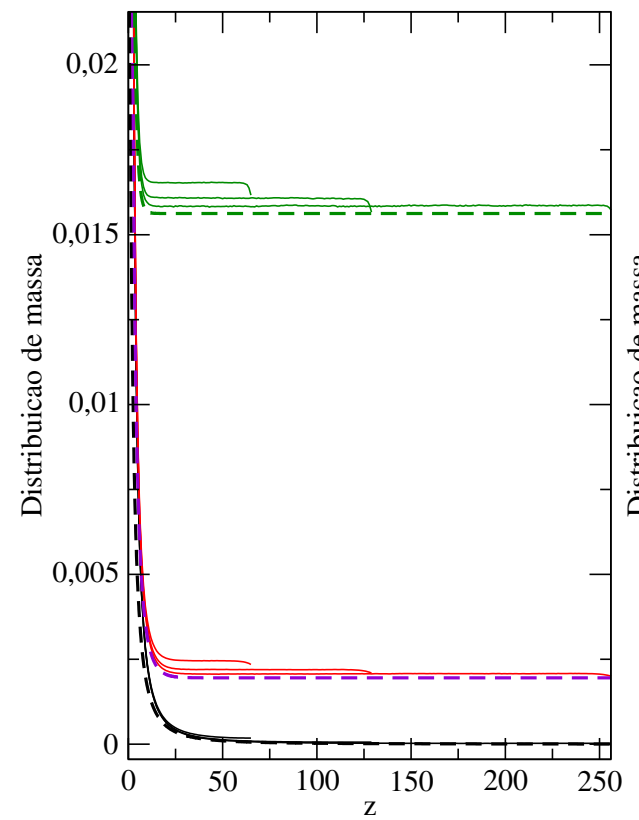

(a) $\sigma=0.2$

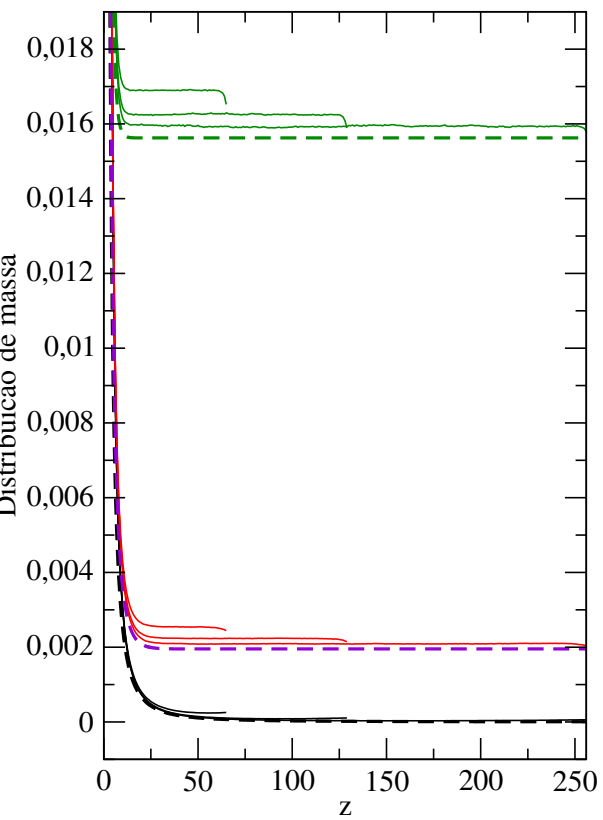

(b) $\sigma=1.0$

Figura 6.2: Distribuição de massa para densidades de íons e contra-íons na direção perpendicular ao plano carregado. Soluções com densidades $\rho=0, \rho=0,0009766$ e $\rho=0,0078125$ de sal. Para sistemas com $20 \%$ (a) e 100\% (b) da placa ocupada por partículas carregadas negativamente. As partículas positivas correspondentes estão soltas na solução. As simulações foram feitas em reticulados paralelepídicos de base $16 \times 16$ e comprimentos 64 , 128 e 256 a temperatura de banho térmico $t=1$. Para as densidades de sal não nulas, observamos o mesmo efeito de borda apresentado na figura 6.3.

Um segundo efeito ocorre próximo a superfície oposta a superfície carregada: observa-se que há uma queda na densidade de massa, para as duas concentrações não nulas.

Realizamos uma pequena investigação do efeito na superfície fechada, considerando apenas a solução de sal, na ausência da placa carregada, isto é, o LRPM com condições periódicas de contorno em duas direções e fechado na terceira direção.

Testamos diferentes alcances para os movimentos dos íons na expectativa de reduzir este efeito. A figura 6.3 ilustra o nosso resultado. Observamos 


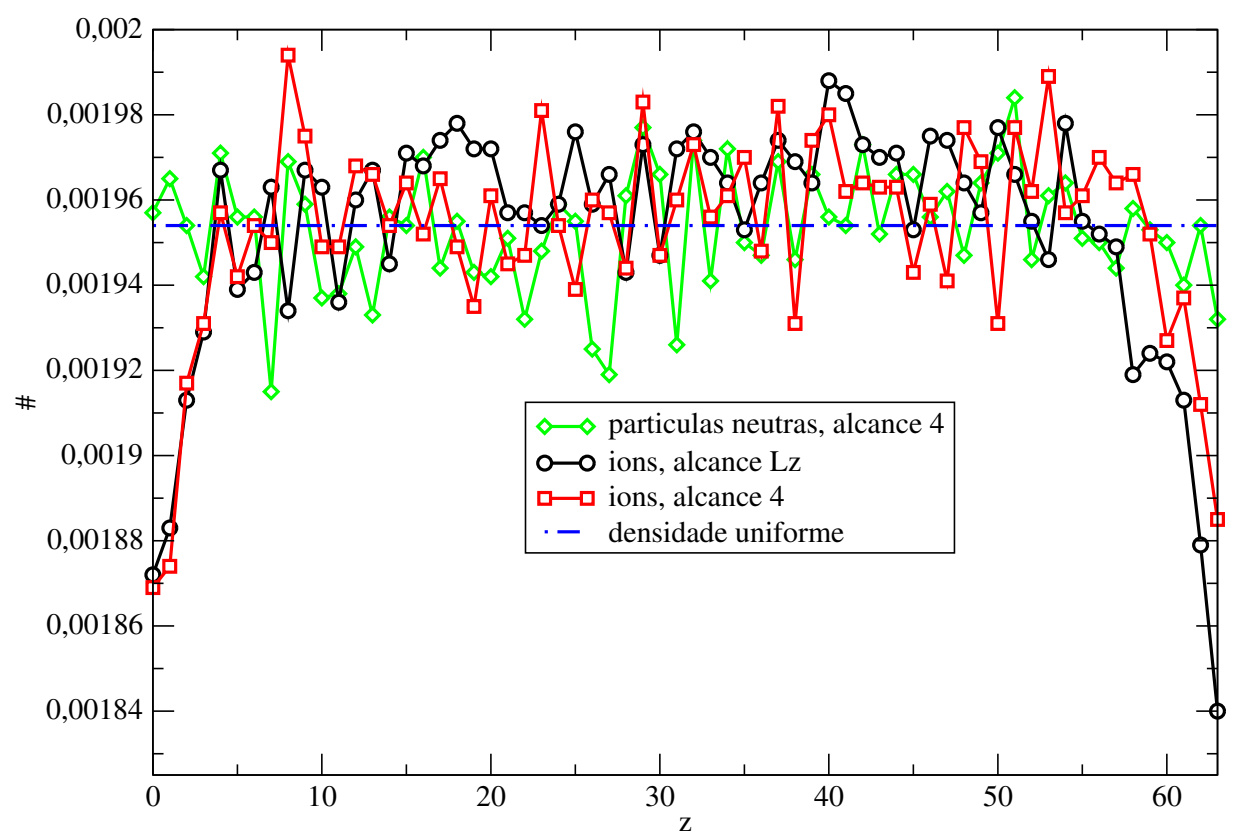

Figura 6.3: Distribuição de massa a longo do eixo z. Concentração de sal $\rho=0,000977$. Neste último caso, foi também simulado o sistema neutro como referência. $t=1, L=16$ e $L_{z}=64$

que, considerando a flutuação estatística, não há diferença entre os dois procedimentos. Na figura, podemos ver também que a distribuição de massa para um sistema de partículas neutras, o gás de rede, não apresenta o efeito de borda e, por isso, concluímos que este é um efeito de superfície devido à carga.

Estamos interessados na influência dos íons da solução nas propriedades térmicas da membrana. Esta influência é atribuída principalmente aos íons próximos à superfície da membrana. Na figura 6.4 apresentamos um detalhe das curvas da figura 6.2. Podemos ver que os diferentes comprimentos $L_{Z}$ da caixa de simulação resultam em distribuições indistinguíveis na região próxima ao plano carregado. Embora este efeito não seja desprezível para o valor do patamar, com foi visto na figura 6.2 , concluímos que caixas de 


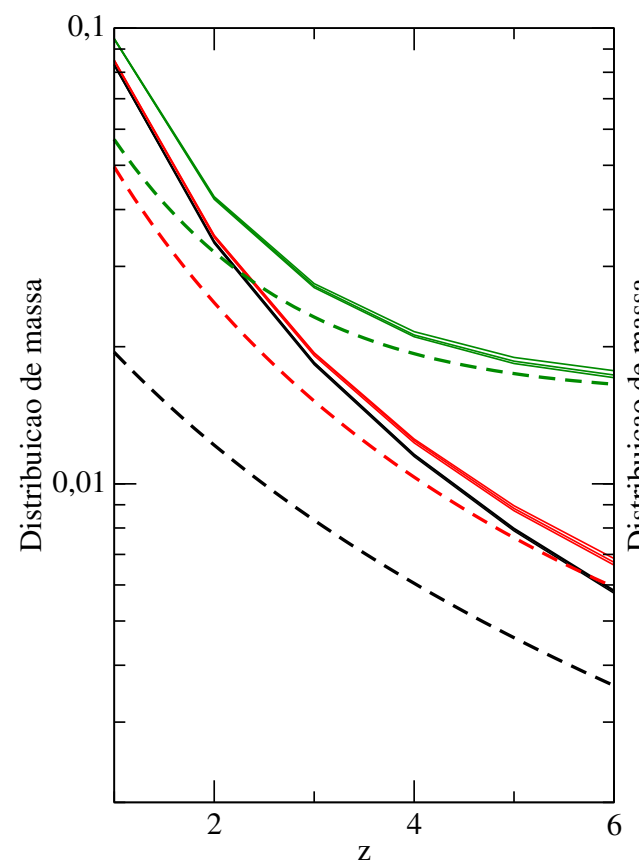

(a) $\sigma=0.2$

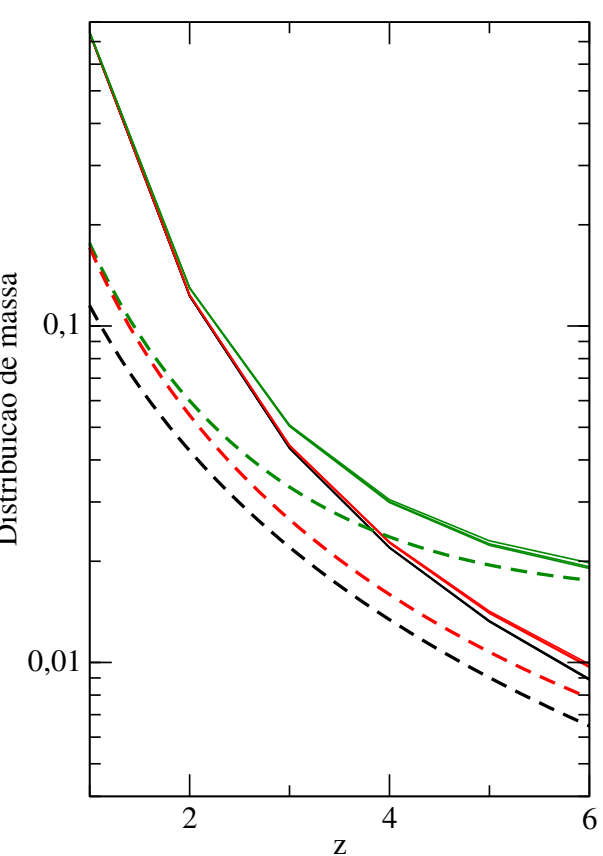

(b) $\sigma=1.0$

Figura 6.4: Distribuição de massa para densidades $\rho=0, \rho=0,0009766$ e $\rho=0,0078125$. Para sistemas com $20 \%$ (a) e $100 \%$ (b) da placa está ocupada por partículas carregadas negativamente. As partículas positivas correspondentes estão soltas na solução. As simulações foram feitas em reticulados paralelepídicos de base $16 \times 16$ e comprimentos 64 , 128 e 256 .

simulação de comprimento 32 e 64 são grandes o suficiente para o resultado que interessa (íons das primeiras camadas de solvatação) ser independente do tamanho da caixa de simulação.

Uma descrição analítica muito utilizada para a distribuição de íons na proximidade de um plano carregado é dada pela distribuição de PoissonBoltzmann [67] para a solução sem sal:

$$
n(z)=\frac{1}{2 \pi \tilde{l}_{B}} \frac{1}{\left(z+\frac{1}{\pi \tilde{\sigma} \tilde{l}_{B}}\right)}
$$


$\mathrm{e}$

$$
n_{ \pm}(z)=n_{0}\left(\frac{1+\gamma \mathrm{e}^{-k z}}{1-\gamma \mathrm{e}^{-k z}}\right)^{\mp 2}
$$

onde

$$
\begin{gathered}
\tilde{l}_{B}=\lambda \beta \frac{1}{4 \pi}, \\
k=\sqrt{8 \pi \tilde{n}_{0} \tilde{l}_{B}}, \\
\tilde{\sigma}=\sigma \frac{a^{2}}{e}
\end{gathered}
$$

é a densidade superficial adimensional de carga, a é a largura do sítio na rede, e a carga do elétron,

$$
\gamma=-\sqrt{\frac{2}{\pi} \frac{\tilde{n}_{0}}{\tilde{\sigma}^{2} \tilde{l}_{B}}}+\sqrt{1+\frac{2}{\pi} \frac{\tilde{n}_{0}}{\tilde{\sigma}^{2} \tilde{l}_{B}}}
$$

$\tilde{n}_{0}$ é a densidade adimensional de sal na solução.

As curvas dessa distribuição para o sistema simulado estão representadas por meios de linhas tracejadas nas figuras $6.4 \mathrm{a}$ e 6.4b. Podemos verificar nessas figuras, que a diferença entre os resultados da simulação e o resultado analítico é grande na região de interesse e só se torna desprezível longe do plano carregado.

Na figura 6.5 apresentamos a distribuição de massa para diversas temperaturas. A temperatura é definida de maneira tal que $t=\frac{1}{\lambda} \frac{1}{\beta}$. Para temperaturas baixas, um decaimento mais acentuado é observado, como esperado 


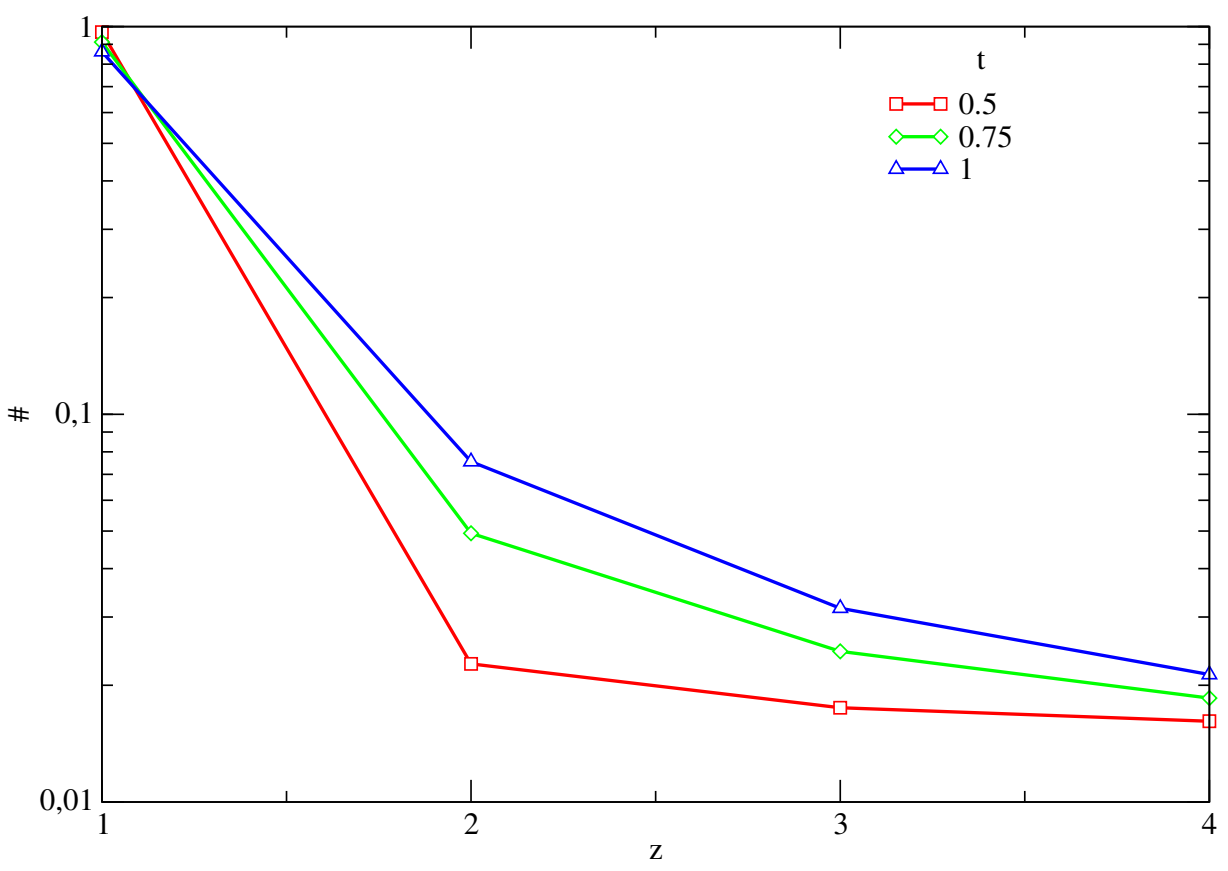

Figura 6.5: Distribuição de massa a longo do eixo $z$ para diversas temperaturas. Concentração de sal $\rho=0,0078125, L_{z}=256, L=16$ e a placa está totalmente carregada.

\subsection{Resultados exploratórios: solução lipídica sem sal}

Obtivemos alguns resultados de simulação para os dois modelos: DLG dipolar

e DLG carregado, sem adição de sal.

As simulações foram realizadas no ensemble canônico, a temperatura e quantidade de partículas são fixas. O potencial químico foi calculado de acordo com o método de Widom. Como vimos, este método estima o potencial químico através da diferença de energia ao inserir virtualmente uma partícula. Para cada espécie de partícula, existe um potencial químico associado. No modelo DLG dipolar, existe apenas uma espécie de partícula, o lipídio associado, e o potencial químico é único. No modelo carregado, 
o lipídio dissociado, o lipídio associado e o contraíon positivo são espécies diferentes. Neste estudo, calculamos apenas o potencial químico do lipídio associado.

Nas figuras 6.6a e 6.6b, apresentamos isotermas de potencial químico pela densidade para os sistemas dipolar e carregado, respectivamente. Os patamares determinam o potencial químico onde ocorre a transição de fase, neste potencial químico observamos o salto de densidade, associada a um salto da área por lipídio. Nas figuras 6.6c e 6.6c, apresentamos isotermas de potencial químico por "magnetização". Os patamares nas curvas por magnetização correspondem aos mesmos valores das curvas por densidade. A transição de ordem-desordem ocorre junto com a transição de densidade. Reconhecemos uma fase ordenada, de densidade alta, que transiciona para uma fase desordenada, de densidade menor. O comportamento dos dois modelos, dipolar e carregado, é semelhante. Observando-se apenas uma maior dispersão dos potenciais químicos de coexistência no caso do modelo carregado.

No caso do modelo carregado, além das descontinuidades na densidade e na ordem das cadeias, ocorre também uma descontinuidade na densidade de carga da membrana. Na figura 6.7 apresentamos a carga média por lipídio do modelo carregado em diferentes temperaturas. Pode-se verificar que a densidade da carga na membrana diminui a medida que a densidade de lipídios aumenta. A figura também mostra que a densidade de carga para uma dada densidade de lipídio aumenta com a temperatura.

A inspeção da figura 6.6b mostra que, em $t=0,6$, por exemplo, as duas densidade de coexistência são 0,45 e 1. Da figura 6.7, este salto na densidade corresponde a um salto na densidade de carga de 0,28 para 0,15. Em labo- 


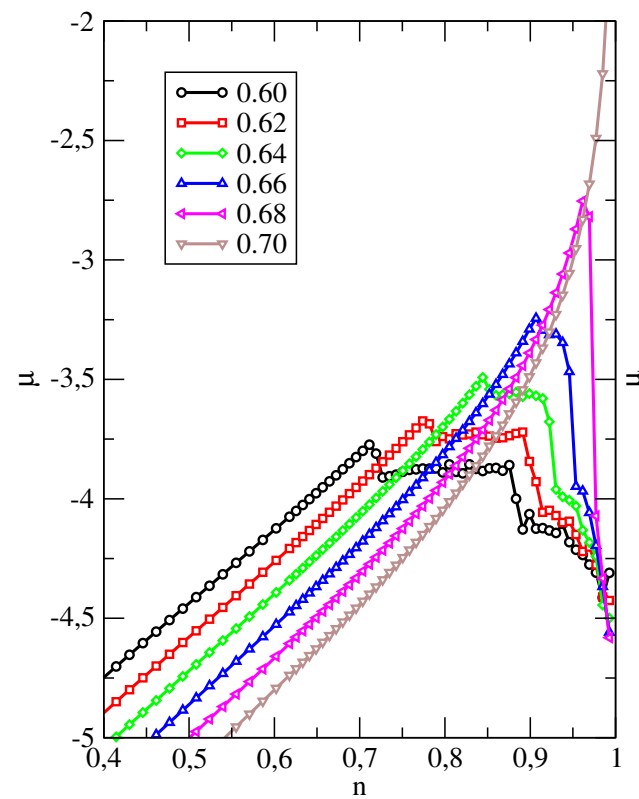

(a) DLG dipolar

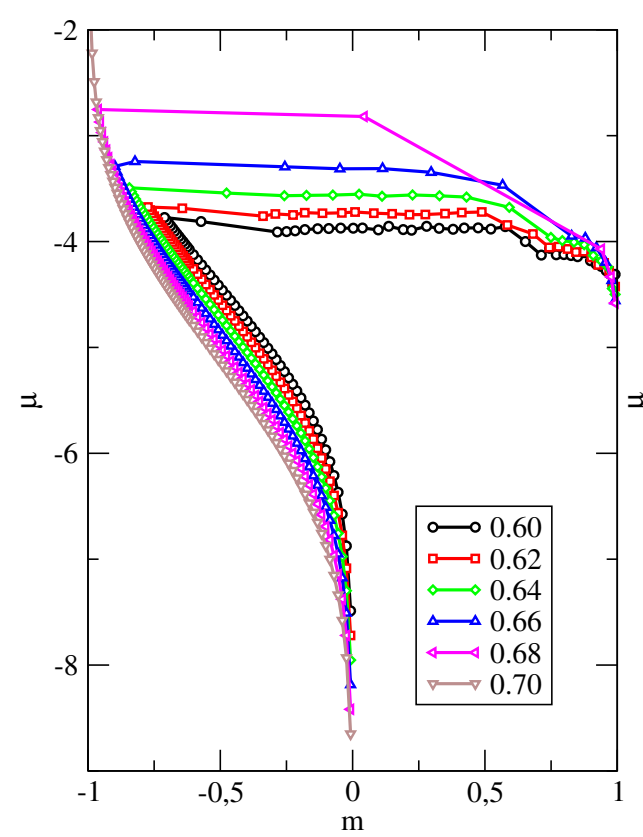

(c) modelo neutro

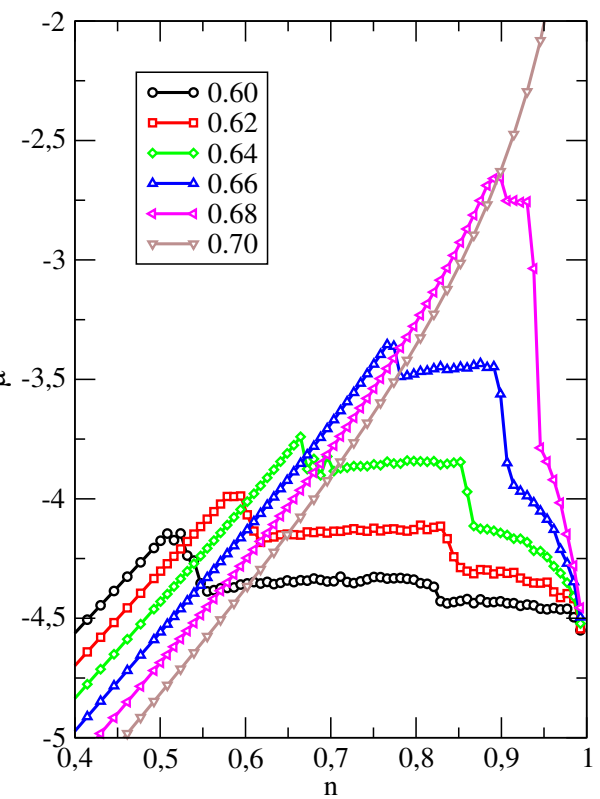

(b) modelo carregado

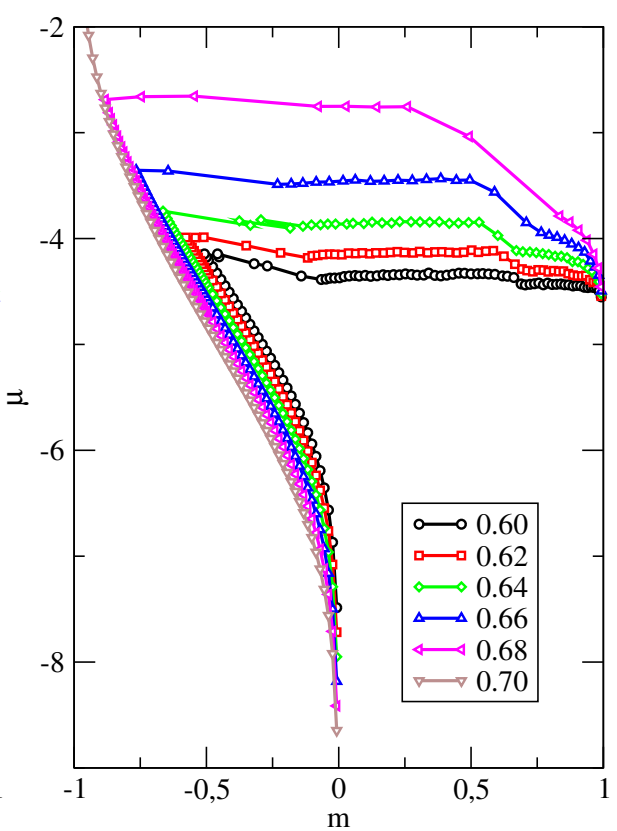

(d) modelo carregado

Figura 6.6: Isotermas de potencial químico por densidade, (a) e (b), e potencial químico por "magnetização", (c) e (d). Para o modelo DLG carregado, (b) e (d), e o modelo DLG dipolar, (a) e (c). $K / J=1, \Delta / J=0.6, \Omega=1000$ e $\lambda / J=0.5$ A temperatura de cada isoterma é indicada na legenda. 


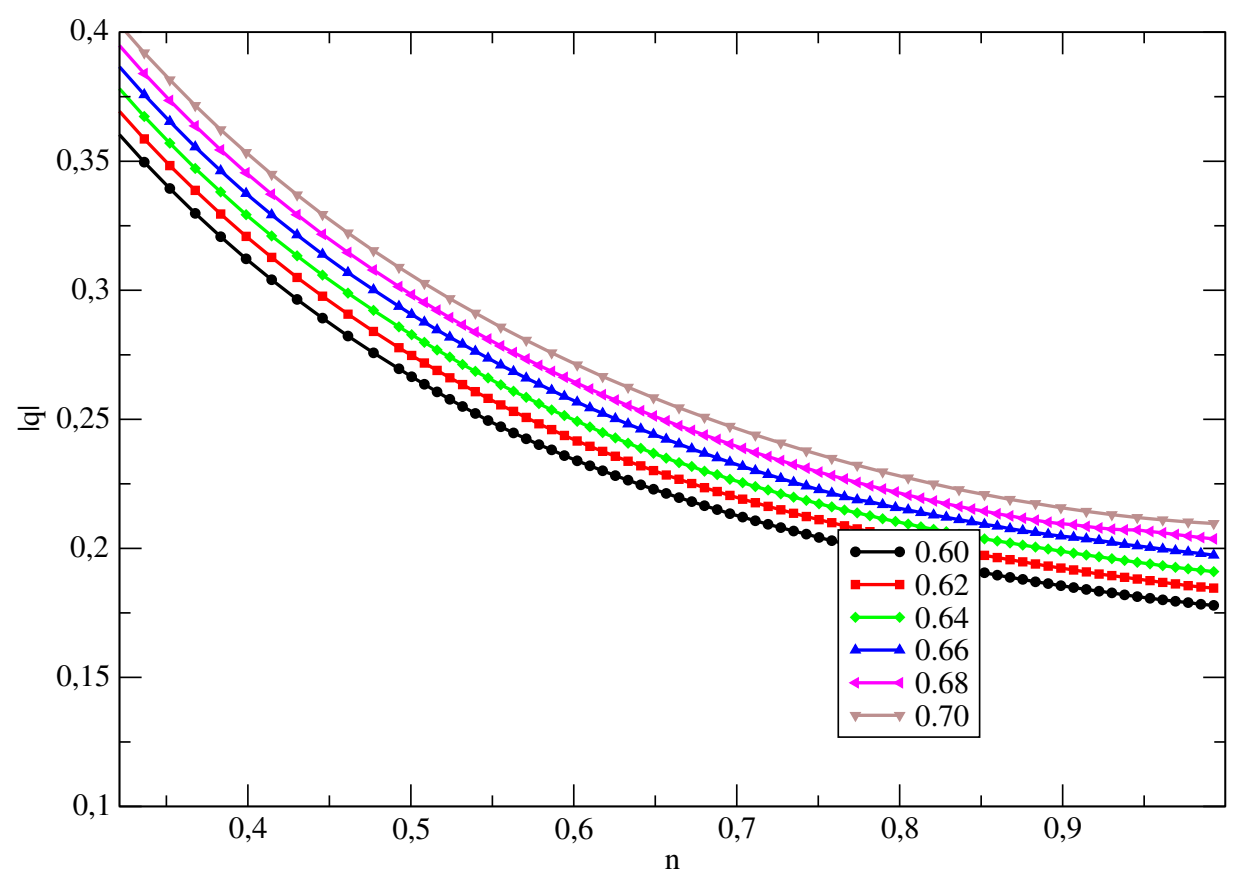

Figura 6.7: Isotermas da carga média por lipídio do modelo carregado em função da densidade superficial de lipídios, $n . K=1, \Delta=0.6, \Omega=1000$ e $\lambda=0.5$.

ratório, se obtém indiretamente[10] medidas de ionização da membrana, isto é, da quantidade de íons que a membrana liberou na solução. Estas medidas mostram que há um acréscimo da ionização na transição ordem-desordem.

Podemos obter alguns pontos dos diagramas de fases temperatura - potencial químico dos dois modelos, o modelo dipolar e o modelo carregado.

Na figura 6.8, comparamos o diagrama de fases do modelo neutro (DLG) e o modelo dipolar. Neste diagrama apresenta-se a linha de coexistência entre as fases ordenada e desordenada. O potencial químico foi renormalizado pelo módulo do potencial químico da transição a temperatura nula, $\mu_{\mathrm{DLG}}^{*}(T=$ $0)=-6,4$ e $\mu_{\mathrm{DLGD}}^{*}(T=0)=-3,63$, respectivamente (ver o conjunto de equações 3.43 e 6.1, para sistema completamente ordenado). Pode-se ver que a linha de transição para o modelo neutro é muito mais abrupta do que a 


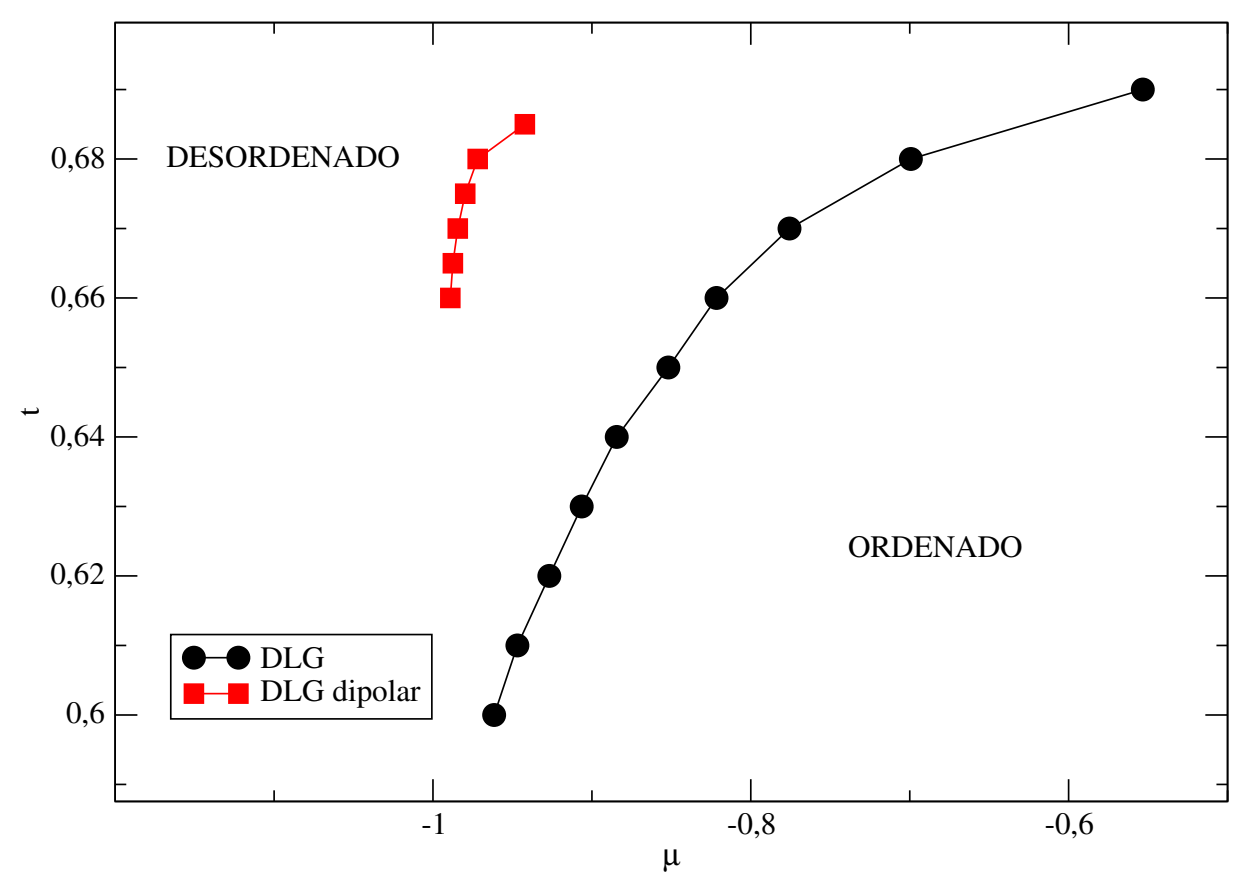

Figura 6.8: Diagrama de fase temperatura-potencial químico para o modelo DLG e o modelo $D L G$ dipolar. $O$ fim da linha não representa ponto crítico. $K / J=1, \Delta / J=0.6$, $\Omega=1000$ e $\lambda / J=0.5$.

linha correspondente para o modelo dipolar. A repulsão entre as cargas da cabeça polar torna a fase ordenada menos estável.

A figura seguinte, 6.9, ilustra a diferença entre as linhas de coexistência do modelo dipolar e do modelo carregado. Pode-se observar na figura que a temperatura de transição ordem - desordem é maior para o modelo carregado em potenciais químicos mais baixos, mas a situação se inverte para potenciais químicos mais altos. No sistema experimental, a temperatura de transição da membrana carregada, DMPG (fig. 1.5b), é menor que a temperatura da membrana neutra, DMPC (fig. 1.2b). Os comportamentos dos sistemas experimentais e dos nossos modelos divergem para baixo potencial químico. Isto poderia ser decorrente da ausência de uma representação da água, pois os dipolos da água certamente desempenham um papel importante 


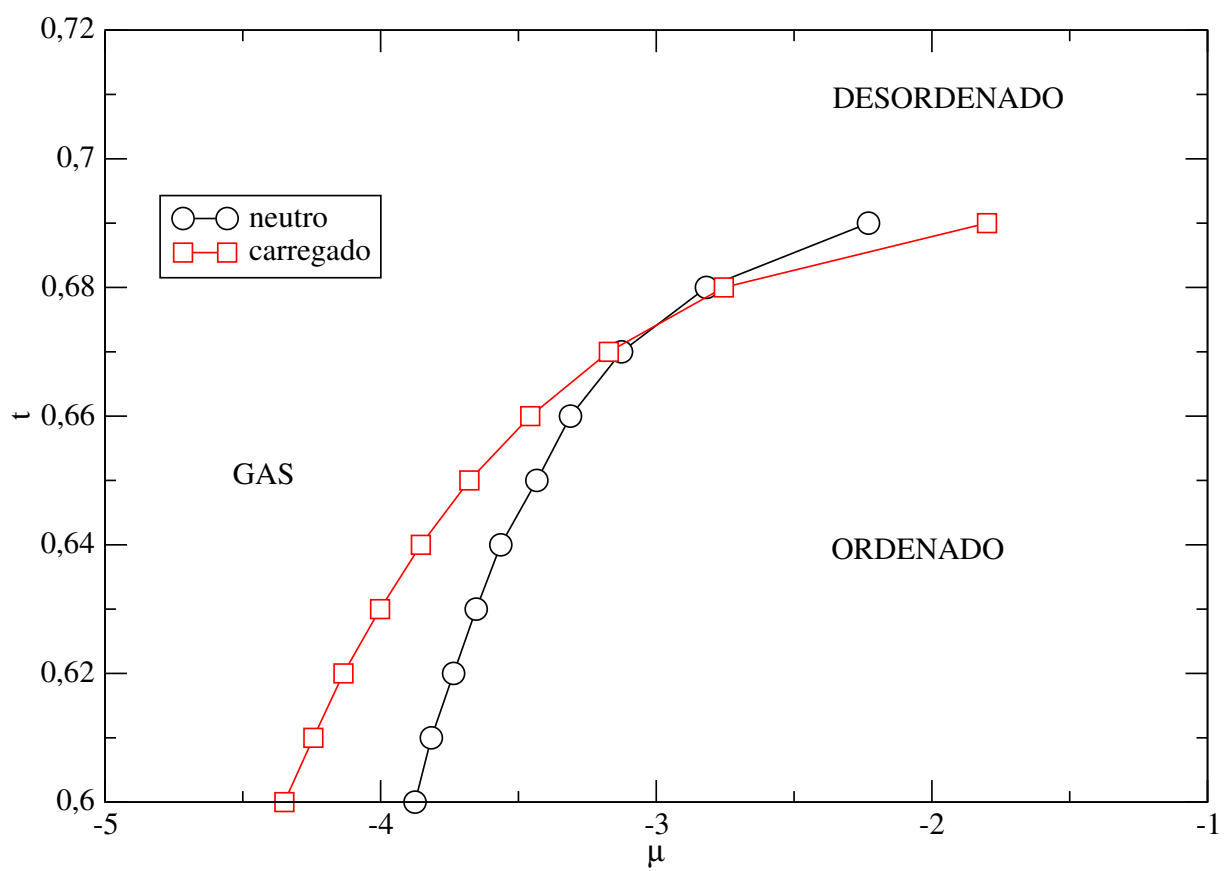

Figura 6.9: Diagrama de fase temperatura-potêncial químico (renormalizado) para o modelo DLG carregado e o modelo DLG dipolar. Os finais das linhas não representam pontos críticos. $K / J=1, \Delta / J=0.6, \Omega=1000$ e $\lambda / J=0.5$.

na organização do sistema real.

\section{Pressão constante}

Analisamos alguns dos nossos resultados em termos de uma pressão lateral constante. Para o modelo DLG carregado, temos três espécies de partículas. Portanto, a relação de Gibbs-Duhem se escreve

$$
+A d \Pi-N_{0} d \mu_{0}-N_{-} d \mu_{-}-N_{+} d \mu_{+}=0,
$$

para temperatura e pressão hidrostática constantes. $N_{0}$ é a quantidade de lipídios associados, $N_{-}$é a quantidade de lipídios dissociados, $N_{+}$é a quantidade de contraíons positivos. As duas últimas grandezas são nulas para o 
DLG dipolar.

No equilíbrio, os potenciais químicos não são independentes, pois

$$
\operatorname{Lip}^{-}+\text {íon }^{+} \rightarrow \operatorname{Lip}^{0}
$$

leva a

$$
\mu_{0} d N_{0}+\mu_{+} d N_{+}+\mu_{-} d N_{-}=0 \quad \text {. }
$$

Além disso, $d N_{+}=d N_{-}=-d N_{0}$. Portanto,

$$
\mu_{0}=\mu_{+}+\mu_{-} .
$$

A conservação de partículas do sistema todo também permite escrever $N_{+}=$ $N_{-}$e $N_{+}+N_{0}=N$. Reunindo todas estas condições, obtemos

$$
A d \Pi-N d \mu_{0}=0 \quad .
$$

Portanto,

$$
\Pi(t, \mu)=\int_{-\infty}^{\mu} n\left(t, \mu^{\prime}\right) d \mu^{\prime} .
$$

Utilizamos a expressão acima para obter a pressão lateral correspondente ao potencial químico a cada ponto da isoterma.

Na figura 6.10 apresentamos resultados para a área por cabeça polar e a densidade superficial de carga em função da temperatura, à pressão lateral fixa. A área relativa é a área ocupada pelo lipídio dividida pela área ocupada do lipídio no estado ordenado. Definimos ionização como a quantidade de carga que a membrana libera para a solução. Como o íon liberado tem carga 


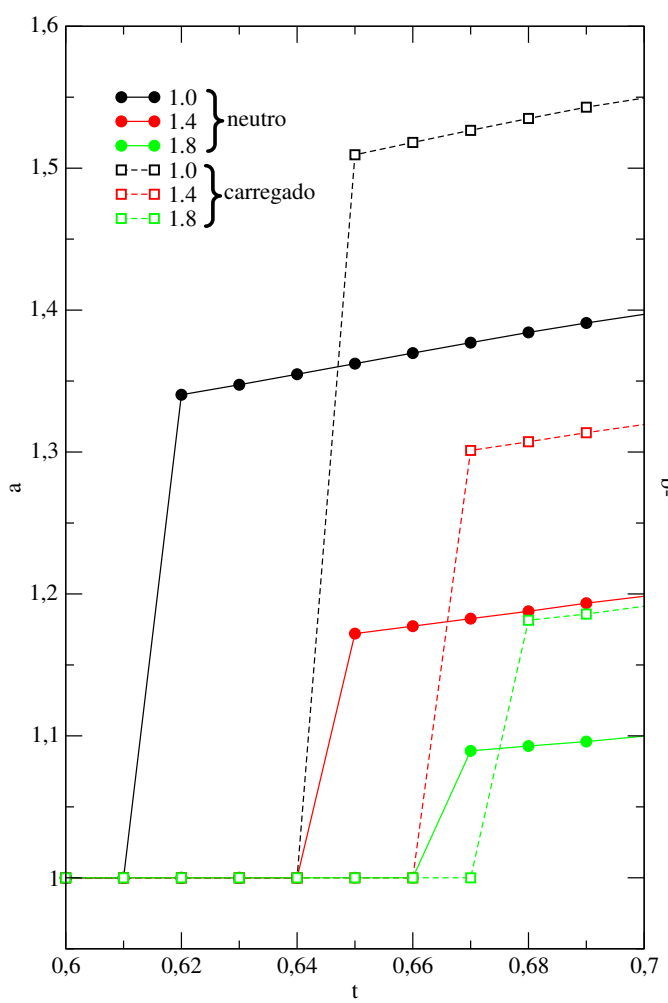

(a) Área relativa média por lipídio

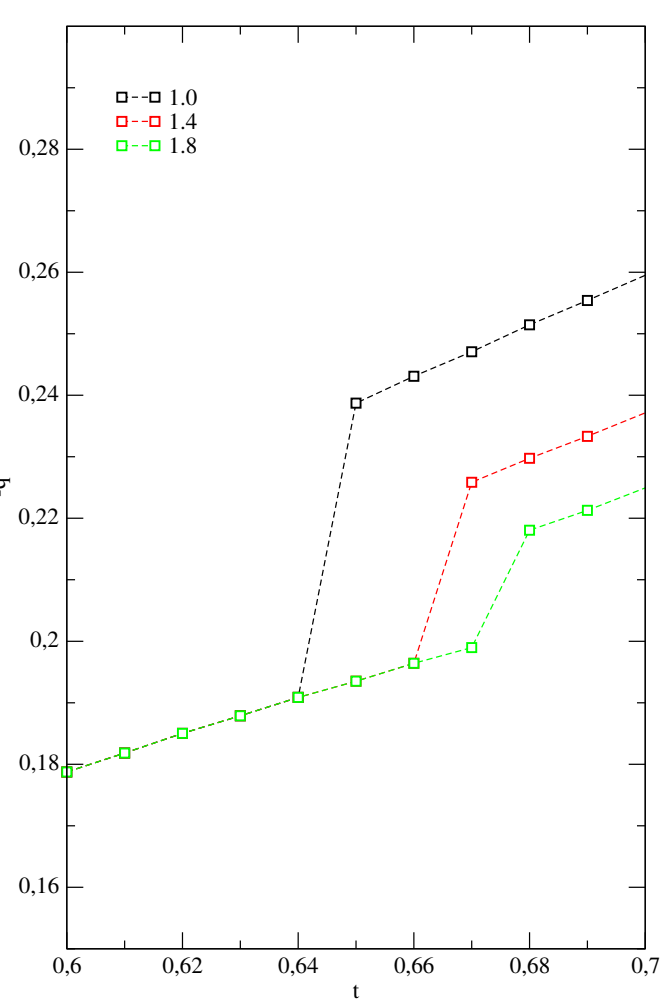

(b) Ionização média por lipídio

Figura 6.10: (a) Área relativa média por lipídio em função da temperatura, a pressão fixa. Modelo dipolar (símbolos cheios) e modelo carregado (símbolos vazios), para diferentes pressões $(1,1,4$ e 1,8). (b) Ionização média por lipídio em função da temperatura, nas mesmas pressões da figura (a). $K / J=1, \Delta / J=0.6, \Omega=1000 \mathrm{e} \lambda / J=0.5$.

positiva, a carga da cabeça polar se torna negativa. Nas pressões escolhidas observamos um salto na carga entre $10 \%$ e $30 \%$

A comparação desses resultados com resultados de sistemas experimentais não é tão direta como no caso das monocamadas de Langmuir, para as quais a pressão lateral é medida no experimento. No caso das membranas, não sabemos como se pode medir a pressão lateral. Na literatura, ainda não há consenso sobre a interpretação dessa pressão interna à vesícula. 


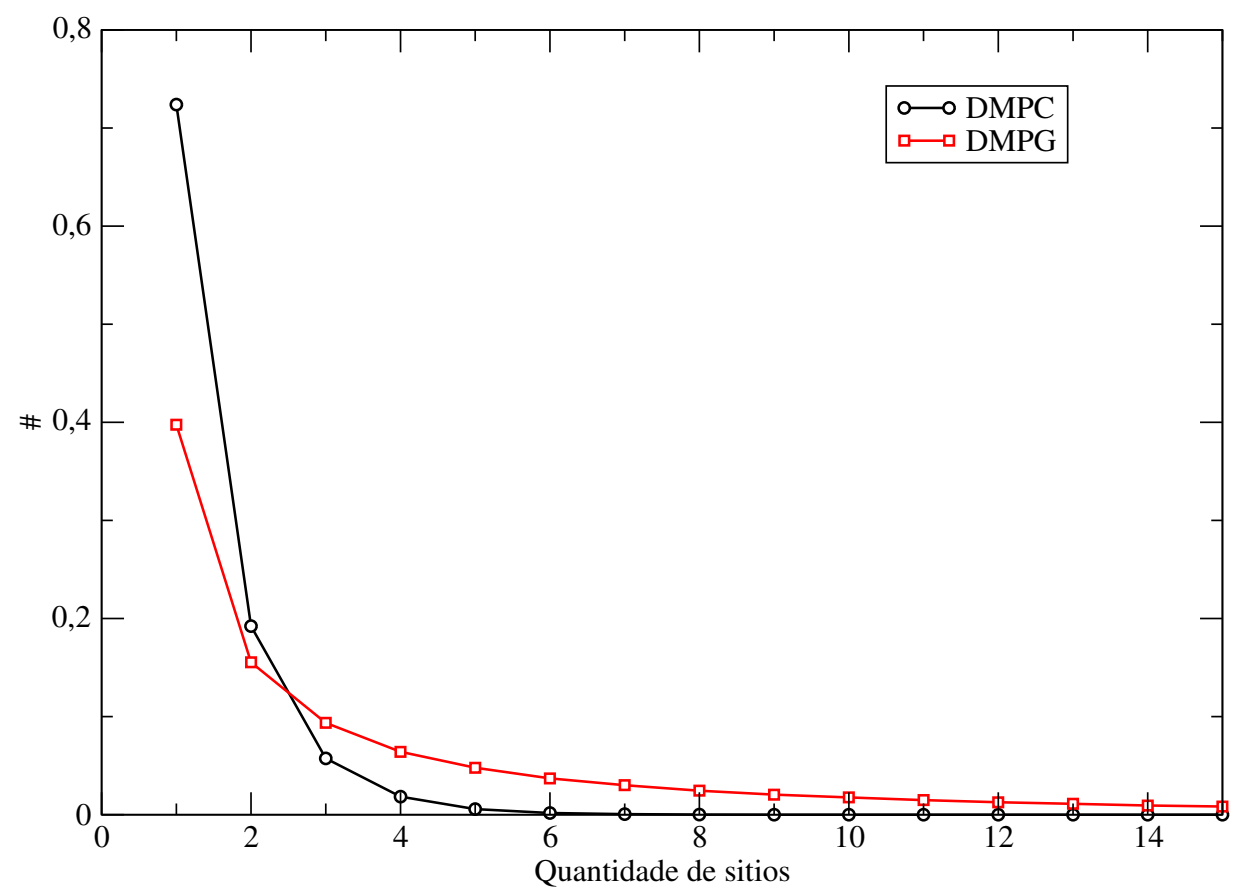

Figura 6.11: Distribuição de buracos na superfície para temperatura $t=0,68$ e densidade $n=972 / 1024=0,95$. Os buracos do sistema carregado tem tamanho médio de 4,45, maiores que os do sistema neutro 1,4. $L=32, L_{z}=64, K / J=1, \Delta / J=0,6, \Omega=1000 \mathrm{e}$ $\lambda / J=0,5$.

\section{Estatística de buracos}

Trabalhos recentes $[6,9,68,69,70]$ que comparam a mudança de tamanho de vesículas carregadas e neutras, através de espalhamento de luz, propõem a formação de buracos na superfície da vesícula carregada na transição ordem desordem. Motivados por estes trabalhos, decidimos medir a distribuição do tamanhos de buracos para os dois modelos, DLG carregado e DLG dipolar.

Definimos como buraco o aglomerado de sítios vazios. Consideramos que um sítio faz parte de um determinado buraco se estiver no estado vazio e se for primeiro vizinho de um sítio desse buraco.

Na figura 6.11, apresentamos a distribuição de tamanho de buracos na superfície da membrana, em uma região de coexistência das fases ordenada 
e desordenada, à mesma temperatura, $t=0,68$. Observa-se que o tamanho médio dos buracos do modelo DLG carregado é da ordem de três vezes maior que a média do tamanhos dos buracos do modelo DLG dipolar.

Isto é compatível com a ideia que a força repulsiva entre lipídos carregados é maior. No entanto, um estudo sistemático dessas distribuições é necessário para investigar esta hipótese, sugerida pelos trabalhos experimentais. Nosso modelo pode servir de um teste importante da hipótese. 


\section{Capítulo 7}

\section{Conclusões e considerações}

\section{finais}

Neste trabalho desenvolvemos três modelos para a transição de fase ordemdesordem de agregados lipídicos.

Baseados no modelo de Doniach, introduzimos flutuação na distância entre lipídios, resultando o modelo DLG. Isto nos permitiu associar a transição ordem-desordem com o aumento da área superficial de membranas de maneira menos pictórica. Felizmente, este era o elemento faltante para termos um modelo simplificado que descrevesse a transição ordem-desordem, tanto de membranas, como de monocamadas de Langmuir. Isto é um indício de que este modelo é um aprimoramento em relação ao modelo de Doniach e abre novas possibilidades. Seria muito interessante poder ajustar os parâmetros do modelo com dados experimentais, tanto para monocamadas de Langmuir como para membranas. A possibilidade de usar o modelo para relacionar estes dois sistemas é animadora. 
A distância entre os lipídios no modelo DLG é definida, permitindo a inclusão de interações Coulombianas e, resultando em um segundo e um terceiro modelos, respectivamente, DLG dipolar e DLG carregado. Nestes modelos, a cabeça polar é representada por um par neutro de íons carregados e a interação Coulombiana é tratada explicitamente. Estes modelos foram desenvolvidos para estudar o efeito da dissociação sobre as transições de fases em membranas em solução, e por isso, estes modelos são acoplados a um modelo de solução iônica (LRPM). Alias, o modelo DLG dipolar representa aglomerados de lipídios zwitteriônicos e o modelo DLG carregado representa camadas de lipídios iônicos.

Em todos os modelos de membranas, observamos a transição principal, ordem - desordem, associada a um aumento da área média por lipídio. No modelo DLG carregado, esta transição está associada também a uma descontinuidade da densidade de carga da membrana, em consonância com sistemas experimentais.

O modelo DLG carregado está bem definido. As dificuldades em relação à simulação foram esclarecidas e resolvidas. Realizamos um estudo exploratório de suas propriedades, que sugere que o modelo é promissor para o esclarecimento do comportamento peculiar de membranas formadas por lipídios carregáveis.

Como subproduto desse estudo, construímos uma metodologia para a simulação de sistemas com interação de longo alcance, mesmo na presença de anisotropia. Desenvolvemos um algoritmo para o cálculo de diferenças de energia que é simples, e, além de tornar mais eficientes os algoritmos disponíveis na literatura, não restringe a escolha das condições de contorno. 


\section{Referências Bibliográficas}

[1] Donald Voet and Judith G. Voet. Biochemistry 1. Wiley \& Sons, 3. a. edition, February 2004.

[2] J. Koolman and K. H. Roehm. Color atlas of biochemistry. Thieme, 2005 .

[3] M. Bloom, E. Evans, and O. G. Mouritsen. Physical properties of the fluid lipid-bilayer component of cell membranes: a perspective. Quarterly reviews of biophysics, 24(3):293-397, August 1991.

[4] M. T. Lamy-Freund and K. A. Riske. The peculiar thermo-structural behavior of the anionic lipid DMPG. Chem. Phys. Lipids, 122:19, 2003.

[5] Wikipedia. Lipid bilayer phase behavior — wikipedia, the free encyclopedia, 2009. [Online; accessed 18-April-2010].

[6] Thais A. Enoki, Vera B. Henriques, and M. Teresa Lamy. Light scattering on the structural characterization of dmpg vesicles along the bilayer anomalous phase transition. Chemistry and Physics of Lipids, aceito para publicação. 
[7] Gregor Cevc and Derek Marsh. Phospholipid Bilayer. John Wiley \& Sons, 1987.

[8] Vladimir M. Kaganer, Helmuth Möhwald, and Pulak Dutta. Structure and phase transitions in langmuir monolayers. Rev. Mod. Phys., 71:779819, Apr 1999.

[9] Karin A. Riske, Lia Q. Amaral, and M. Teresa Lamy. Extensive bilayer perforation coupled with the phase transition region of an anionic phospholipid chain ordering in liquid crystals. II. structure of bilayer membranes. Langmuir, 25(17):10083-10091, 2009.

[10] R. P. Barroso, K. A. Riske, V. B. Henriques, and M. T. Lamy. Ionization and structural changes of the dmpg vesicle along its anomalous gel-fluid phase transition: A study with different lipid concentrations. Langmuir, 26(17):13805-13814, 2010.

[11] C. Goldman. J. Chem. Phys., 114:6242 - 6248, 2001.

[12] M. T. Lamy M. N. Tamashiro Vera B. Henriques, R. Germano. Phase transitions and spatially ordered counterion association in ionic-lipid membranes: Theory versus experiment. Langmuir, 27(21):13130-13143, 2011.

[13] J. F. Nagle. Theory of biomembrane phase transitions. The Journal of Chemical Physics, 58(1):252-264, 1973.

[14] S. Doniach. Thermodynamic fluctuations in phospholipid bilayers. The Journal of Chemical Physics, 68(11):4912-4916, 1978. 
[15] Marcelja S. Chain ordering in liquid crystals. II. structure of bilayer membranes. Biochim Biophys Acta., 367(2):165-176, 1974.

[16] David A. Pink and Dennis Chapman. Protein-lipid interactions in bilayer membranes: A lattice model. volume 76, pages 1542-1546, 1979.

[17] O. G. Mouritsen, A. Boothroyd, R. Harris, N. Jan, T. Lookman, L. MacDonald, D. A. Pink, and M. J. Zuckermann. Computer simulation of the main gel-fluid phase transition of lipid bilayers. The Journal of Chemical Physics, 79(4):2027-2041, 1983.

[18] Lars K. Nielsen, Thomas Bjornholm, and Ole G. Mouritsen. Thermodynamic and real-space structural evidence of a $2 \mathrm{D}$ critical point in phospholipid monolayers. Langmuir, 23(23):11684-11692, oct 2007.

[19] István P. Sugár. On the inner structure and topology of clusters in two-component lipid bilayers. comparison of monomer and dimer ising models. The Journal of Physical Chemistry B, 112(37):11631-11642, August 2008.

[20] Lars K. Nielsen, Thomas Bjornholm, and Ole G. Mouritsen. Critical phenomena: Fluctuations caught in the act. Nature, 404(6776):352-352, mar 2000 .

[21] Julia Preu, Thomas Gutberlet, and Thomas Heimburg. Network formation of DMPG dispersions studied by light scattering, SANS, SAXS and calorimetry. Chemistry and Physics of Lipids, 149(Supplement 1):S40S40, 2007. Abstracts from the 48th International Conference on the Bioscience of Lipids. 
[22] Agnieszka E. Hac, Heiko M. Seeger, Matthias Fidorra, and Thomas Heimburg. Diffusion in two-component lipid membranes-A fluorescence correlation spectroscopy and monte carlo simulation study. Biophysical Journal, 88(1):317-333, 2005.

[23] Vitaliy Oliynyk, Markus Jager, Thomas Heimburg, Vitaly Buckin, and Udo Kaatze. Lipid membrane domain formation and alamethicin aggregation studied by calorimetry, sound velocity measurements, and atomic force microscopy. Biophysical Chemistry, 134(3):168-177, 2008.

[24] Salvatore Chiantia, Jonas Ries, and Petra Schwille. Fluorescence correlation spectroscopy in membrane structure elucidation. Biochimica et Biophysica Acta (BBA) - Biomembranes, 1788(1):225-233, 2009. Lipid Interactions, Domain Formation, and Lateral Structure of Membranes.

[25] Ronald Dickman and George Stell. Phase diagram of the lattice restricted primitive model. In: AIP. (Org.). Proceedings of the AIP Workshop on Treatment of Electrostatic Interactions in Computer Simulations of Condensed Media., 1999.

[26] M. Blume, V. J. Emery, and Robert B. Griffiths. Ising model for the $\lambda$ transition and phase separation in he3-he4 mixtures. Phys. Rev. A, 4(3):1071-1077, September 1971.

[27] Juha-Matti I. Alakoskela and Paavo K. J. Kinnunen. Thermal phase behavior of DMPG: The exclusion of continuous network and dense aggregates. Langmuir, 23(8):4203-4213, March 2007. doi: 10.1021/la062875i. 
[28] R. Friedberg and J. E. Cameron. Test of the monte carlo method: Fast simulation of a small ising lattice. The Journal of Chemical Physics, 52(12):6049-6058, 1969.

[29] K. Binder. Statistical mechanics of finite three-dimensional ising model. Physica, 62:508-526, 1971.

[30] I. Morgenstern and K. Binder. Magnetic correlations in two-dimensional spin-glasses. Physical Review B, 22(1):288-303, 1980.

[31] Borko Stosic, Sava Milosevic, and H. Eugene Stanley. Exact results for the two-dimensional ising model in a magnetic field: Tests of finite-size scaling theory. Physical Review B, 41(16):11466-11478, 1990.

[32] N. Metropolis, A. W. Rosenbluth, M. N. Rosenbluth, A. H. Teller, and E. Teller. Equation of state calculations by fast computing machines. The Journal of Chemical Physics, 21:1087, 1953.

[33] M. P. Allen and D. J. Tildesley. Computer simulation of liquids. Clarendon Press, New York, NY, USA, 1989.

[34] Daan Frenkel and Berend Smit. Understanding Molecular Simulation. Academic Press, Inc., Orlando, FL, USA, 2001.

[35] D. P. Landau e K. Binder. A Guide to Monte Carlo Simulations in Statistical Physics. Cambridge, 2000.

[36] Terrell L. Hill. Thermodynamics of Small Systems. Dolver, New York, NY, USA, 1994. 
[37] Adam Lipowski. Glassy behaviour and semi-local invariance in ising model with four-spin interaction. Journal of Physics A: Mathematical and General, 30(21):7365, 1997.

[38] B. Widom. Some topics in the theory of fluids. J. Chem. Phys., 39(11):2808-2812, 1963.

[39] Mário J. de Oliveira. Physics Letters, 91A(5):815, 1982.

[40] Terrell L. Hill. Statistical Mechanics. McGraw-Hill Book Company, Inc., New York, 1956.

[41] Fabiano C. Cardoso. Micelização: Diagramas de fase e potencial químico. Tese de Doutorado - Universidade de São Paulo - Instituto de Física — Depto. de Física Geral, São Paulo, 2004.

[42] L. Greengard and V. Rokhlin. A fast algorithm for particle simulations. Journal of Computational Physics, 135(2):280-292, August 1997.

[43] P. P. Ewald. Die berechnung optischer und elektrostatischer gitterpotentiale. Annalen der Physik, 369(3):253-287, 1921.

[44] Abdulnour Y. Toukmaji and John A. Board. Ewald summation techniques in perspective: a survey. Computer Physics Communications, 95(2-3):73-92, June 1996.

[45] Jean Pierre Hansen and Ian R. McDonald. Theory of simple liquids. Academic Press, 1990.

[46] Evgenii V Kholopov. Convergence problems of coulomb and multipole sums in crystals. Physics-Uspekhi, 47(10):965-990, 2004. 
[47] P. Hünenberger. Effect of artificial periodicity in simulations of biomolecules under ewald boundary conditions: a continuum electrostatics study. Biophysical Chemistry, 78(1-2):69-88, April 1999.

[48] S. W. de Leeuw and J. W. Perram. Computer simulation of ionic systems. influence of boundary conditions. Physica A: Statistical and Theoretical Physics, 107(1):179-189, 1981.

[49] S. W. de Leeuw, J. W. Perram, and E. R. Smith. Simulation of electrostatic systems in periodic boundary conditions. II. equivalence of boundary conditions. Proc. Royal Soc. London, 373(1752):57-66, October 1980.

[50] Mihaly Mezei. Periodic boundary conditions: when is $E_{i j} \neq E_{j i}, 2000$. http://www.ccp5.ac.uk/ ftpfiles/ ccp5.newsletters/ 47/ pdf/ mezei2.pdf.

[51] K. Young. Physical condition for elimination of ambiguity in conditionally convergent lattice sums. Journal of Mathematical Physics, 28(2):425-427, 1987.

[52] Abdulnour Y. Toukmaji and John A. Board Jr. Ewald summation techniques in perspective: a survey. Computer Physics Communications, $95(2-3): 73-92,1996$.

[53] W. F. van Gunsteren and et al. Biomolecular modelling: goals, problems, perspectives. Angew. Chem. Int. Ed., 45:4064-4092, 2006.

[54] Paul Tavan Gerald Mathias; Bernhard Egwolf; Marco Nonella . A fast multipole method combined with a reaction field for long-range elec- 
trostatics in molecular dynamics simulations: The effects of truncation on the properties of water. Journal of chemical physics, 118(24):1084710860, 2003.

[55] Lars Onsager. Electric moments of molecules in liquids. J. Am. chem. Soc., 58:1480-1493, 1936.

[56] Jack Sherman. Crystal energies of ionic compounds and thermochemical applications. Chemical Reviews, 11(1):93-170, 1932.

[57] Wagner Gomes Rodrigues Junior. Sistemas carregados: modelos de simulação. Dissertação de Mestrado — Universidade de São Paulo — Instituto de Física - Depto. de Física Geral, São Paulo, 2011.

[58] George B. Arfken and Hans J. Weber. Mathematical Methods For Physicists. Academic Press, June 2005.

[59] David H. Bailey, Jonathan M. Borwein, Vishal Kapoor, and Eric Weisstein. Ten problems in experimental mathematics. American Mathematical Monthly, 113(6):481-509, 2006.

[60] David Borwein, Jonathan M. Borwein, and Keith F. Taylor. Convergence of lattice sums and madelung's constant. Journal of Mathematical Physics, 26(11):2999-3009, 1985.

[61] David Borwein, Jonathan M. Borwein, and Christopher Pinner. Convergence of madelung-like lattice sum. Trans. Amer. Math. Soc., 350:31313167, 1998. 
[62] David H. Bailey. Simon plouffe's inverter: The madelung constant (in absolute value)., 2012.

[63] S. W. de Leeuw, J. W. Perram, and E. R. Smith. Simulation of electrostatic systems in periodic boundary conditions. I. lattice sums and dielectric constants. Proc. Royal Soc. London, 373(1752):27-56, October 1980.

[64] S. W. de Leeuw, J. W. Perram, and E. R. Smith. Simulation of electrostatic systems in periodic boundary conditions. III. further theory and applications. Proc. Royal Soc. London, 388(1794):177-193, July 1983.

[65] John David Jackson. Classical electrodynamics. John Wiley and Sons, 1962.

[66] Tânia Tomé and Mário J. de Oliveira. Dinâmica estocástica e irreversibilidade. edusp, 2001.

[67] Renato Germano Reis Nunes. Transição ordem-desordem em cadeias lipídicas carregadas - modelo estatístico. Tese de Doutorado - Universidade de São Paulo - Instituto de Física - Depto. de Física Geral, São Paulo, 2011.

[68] K. A. Riske, L. Q. Amaral, H. G. Dobereiner, and M. T. Lamy. Biophys. J., 86:3722, 2004 .

[69] R. P. Barroso, K. R. Perez, I. M. Cuccovia, and M. T. Lamy. Aqueous dispersions of dmpg in low salt contain leaky vesicles. Chem. Phys. Lipids, 165:169-177, 2012. 
[70] J. M. Alakoskela, J. M. Parry, and P.K.J. Kinnunem. The intermediate state of dmpg is stabilized by enhanced positive spontaneous curvature. Langmuir, 26(7):4892-4900, 2010. 